\title{
Discovery of a Diaminopyrimidine FLT3 Inhibitor Active against Acute Myeloid Leukemia
}

\author{
Jamie A. Jarusiewicz, ${ }^{\dagger}$ Jae Yoon Jeon, ${ }^{\dagger}$ Michele C. Connelly, ${ }^{\dagger}$ Yizhe Chen, $^{\dagger}{ }^{\S}$ Lei Yang, ${ }^{\dagger}$ Sharyn D. Baker, ${ }^{\ddagger}$ \\ and R. Kiplin Guy*,,,$\S$
} ${ }^{\dagger}$ Department of Chemical Biology and Therapeutics, St. Jude Children’s Research Hospital, 262 Danny Thomas Place, Memphis,
Tennessee 38105, United States
${ }^{\ddagger}$ Division of Pharmaceutics, College of Pharmacy, The Ohio State University, 500 W. 12th Street, Columbus, Ohio 43210, United
States

Supporting Information

ABSTRACT: Profiling of the kinase-binding capabilities of an aminopyrimidine analogue detected in a cellular screen of the St. Jude small-molecule collection led to the identification of a novel series of FMS-like tyrosine kinase 3 (FLT3) inhibitors. Structure-activity relationship studies led to the development of compounds exhibiting good potency against MV4-11 and MOLM13 acute myelogenous leukemia cells driven by FLT3, regardless of their FLT3 mutation status. In vitro pharmaco-

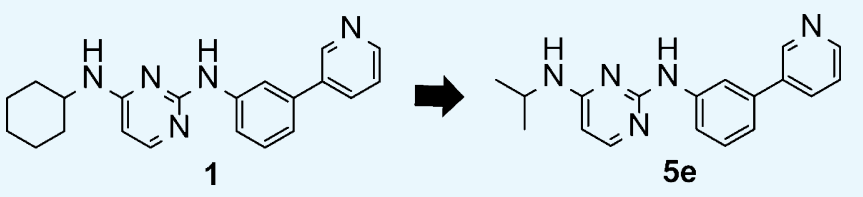

FLT3 $\mathrm{K}_{\mathrm{d}} 61 \mathrm{nM}$

MV4-11 $\mathrm{EC}_{50} 320 \mathrm{nM}$
FLT3 $\mathrm{K}_{\mathrm{d}} 3.6 \mathrm{nM}$

MV4-11 $\mathrm{EC}_{50} 25 \mathrm{nM}$ logical profiling demonstrated that compound 5e shows characteristics suitable for further preclinical development.

\section{INTRODUCTION}

Acute myelogenous leukemia (AML) is characterized by malignant proliferation of hematopoietic cells in the bone marrow, leading to overproduction of abnormally functioning white blood cells. This situation leads to decreased production of red blood cells, infection, and dysfunction of organs. AML occurs in roughly 4 per 100000 individuals; more than half of the reported cases occur in individuals over 65 years of age. ${ }^{1}$ Although the use of hematopoietic stem cell transplants has provided some improved clinical outcomes in AML patients, there has been very little improvement in patient prognosis over the past 20 years. $^{2}$

Normal functioning of FMS-like tyrosine kinase 3 (FLT3), a type III receptor tyrosine kinase, is important for the development and proliferation of hematopoietic stem cells. ${ }^{3,4}$ The binding of the FLT3 ligand to this transmembrane protein causes dimerization and subsequent FLT3 autophosphorylation, which then triggers the activation of several signaling cascades, including the RAS, SRC, and STAT5 pathways. ${ }^{5-8}$ Constitutive activation of FLT3 leads to dysregulated cellular proliferation of hematopoietic cells, and nearly one-third of AML patients have mutations in the FLT3 gene. ${ }^{9}$ Two classes of mutations are commonly found in FLT3: an internal tandem duplication (ITD) located in the juxtamembrane domain, which is the most common, and point mutations at or near residue Asp835. ${ }^{10-14}$ Typically, an AML patient's prognosis is worse if he/she possesses the FLT3-ITD mutation compared to that for patients having normal levels of wild-type FLT3 (wt-FLT3). ${ }^{15,16}$

Initially, kinase inhibitors developed for solid tumors were investigated as FLT3 inhibitors. Several of these inhibitors, including midostaurin, ${ }^{17-19}$ lestaurtinib, ${ }^{20-22}$ crenolanib, ${ }^{23-26}$ tandutinib, ${ }^{27-29}$ sunitinib, ${ }^{30-32}$ and sorafenib, ${ }^{33-36}$ have been evaluated in clinical trials. These compounds tended to inhibit multiple tyrosine kinases, thus leading to toxicity due to off-target effects. ${ }^{37}$ Subsequently, quizartinib and crenolanib were developed as more selective FLT3 inhibitors. ${ }^{38-42}$ The clinical response to FLT3 inhibitors often persists only for a short duration, with acquired point mutations in FLT3 that impact the binding of the inhibitors driving the limited response. ${ }^{37,43,44}$ In particular, FLT3 Asp835 mutants tend to be resistant to "type II" kinase inhibitors that bind to an inactive conformation of the enzyme, wherein the inhibitor is able to make contacts within an allosteric pocket adjacent to the ATP site due to the Asp-Phe-Gly (DFG) motif in the activation loop adopting a conformation in which it is flipped out relative to its active conformation. ${ }^{45-48}$ However, treatment with different FLT3 inhibitors can lead to alternate sets of acquired mutations, and some "type I" ATPpocket-binding inhibitors, which bind within the ATP site but do not reach into the allosteric pocket and do not rely on specific DFG motif conformations, such as crenolanib, are able to bind selectively to FLT3 and also retain their activity against FLT3 Asp835 mutants. ${ }^{23,49,50}$ Consequently, the development of additional new FLT3 inhibitors that can retain their activity against commonly acquired mutations or the use of FLT3 inhibitors in combination therapies may be potential methods to circumvent the problem of resistance.

While investigating potential compounds of interest identified during a cellular high-throughput phenotypic screen for a brain tumor project (results not yet published), the kinase-binding profile of one of the hits suggested potential use as a FLT3

Received: February 8, 2017

Accepted: April 19, 2017

Published: May 10, 2017 
inhibitor. We therefore synthesized a series of compounds based on this hit for evaluation against FLT3 and investigation of their activities in AML cell lines. Herein we describe the structureactivity (SAR) and structure-property relationships resulting from this series of molecules.

\section{RESULTS AND DISCUSSION}

Preliminary Profiling of the Hit (1). The kinase-binding profile of 1 was evaluated using the DiscoveRx KINOMEscan panel of 468 kinases, with ligand competition being measured at a single inhibitor concentration of $\mathbf{1}(10 \mu \mathrm{M}) \cdot{ }^{51-53}$ Selectivity was evaluated through a comparison of the number of nonmutant kinases with which $\mathbf{1}$ interacted relative to the total number of nonmutant kinases tested. Compound 1 reduced ligand binding by $>90 \%$ for 31 kinases of 403 nonmutant kinases tested. Within that set, seven had activity reduced to $>99 \%$ of that of the control. As depicted in Figure 1, $\mathbf{1}$ bound with the highest affinity to

a<smiles>c1cncc(-c2cccc(Nc3nccc(NC4CCCCC4)n3)c2)c1</smiles>

1

b

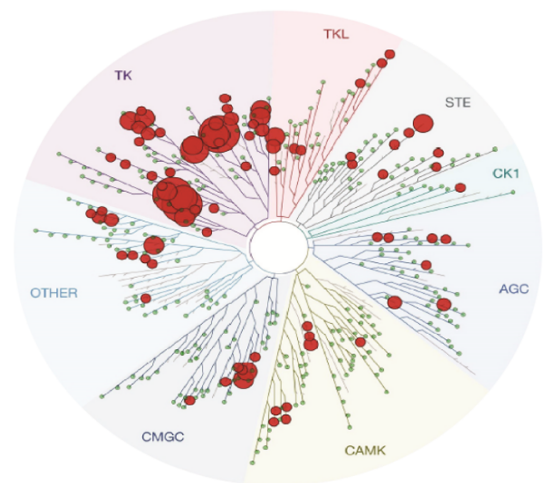

Percent Control

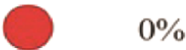

$0.1 \%$

$0.1-1 \%$

$1-5 \%$

- $5-10 \%$

$10-35 \%$

$>35 \%$

Figure 1. Lead series analogue 1. (a) Structure of 1. (b) Kinase profiling of 1 . Kinase tree image showing relative inhibition of ligand binding to 468 human kinases, with the size of the circle being proportionate to the $\%$ inhibition at a fixed dose of $10 \mu \mathrm{M}$.

kinases in the tyrosine kinase family. Further information on the kinase-binding profile is found in Table S1. Subsequently, the $K_{\mathrm{d}}$ values were determined for kinases in which compound 1 had blocked the binding of ligand by $>90 \%$, revealing that 1 bound with a high affinity to FLT3 $(61 \mathrm{nM})$ and a significant but lower affinity to PDGFRB, the JAK1 JH2 domain, and JAK2 JH1 domain (Table 1). Eleven of the kinases had binding affinities within tenfold of those for the FLT3 primary target, which is a reasonably selective binding profile. For instance, previously studied kinase inhibitors, such as the AKT1 inhibitor GSK690693, the IGF1R inhibitor GSK-1838705A, and the mammalian target of rapamycin inhibitor PP-242, had similar relative selectivity profiles. ${ }^{54} \mathrm{We}$ also verified that compound $\mathbf{1}$ inhibited the enzymatic activity of FLT3. As measured using the Z'LYTE kinase inhibition assay (ThermoFisher), 1 was a potent inhibitor of the FLT $3^{\mathrm{WT}}$ enzyme $\left(\mathrm{IC}_{50}=32 \mathrm{nM}\right.$; $95 \%$ confidence interval (CI 95), 24-43 nM). ${ }^{55}$ Compound 1 was also a reasonably potent inhibitor of proliferation for the wellcharacterized MV4-11 AML cell line, which carries an FLT3ITD mutation $\left(\mathrm{EC}_{50}=320 \mathrm{nM}\right.$; CI 95, 160-650 nM). This finding suggested that $\mathbf{1}$ acts on FLT3-ITD in these cells. Although aminopyrimidines have been well studied as kinase inhibitors, low-molecular-weight structures that include an aliphatic moiety, such as $\mathbf{1}$, are not as widely reported and, to the best of our knowledge, have not been described specifically as FLT3 kinase inhibitors. ${ }^{56,57}$ Taken together, the promising activity profile and fairly novel structural character of 1 prompted us to explore additional SARs.

Chemistry. We developed a general route for the synthesis of the majority of the compounds discussed herein (Scheme 1) on the basis of prior work on Aurora kinase inhibitors. ${ }^{58}$ The route, which relied upon sequential additions of an aliphatic amine and various anilines to 2,4-dichloropyrimidine, facilitated systematic variation of the amine substituents at the 2 - and 4-positions of the pyrimidine ring. Whereas the first substitution using an aliphatic amine typically proceeded facilely at either $0{ }^{\circ} \mathrm{C}$ or room temperature ( $\mathrm{rt}$ ), subsequent reactions with the anilines required increased temperature and catalytic $\mathrm{HCl}$. Anilines were obtained either from commercial vendors or were synthesized via the Suzuki reaction or other known procedures (Schemes S1S6). ${ }^{59,60}$ To help define the minimal pharmacophore, we investigated the impact of removing nitrogen atoms from the scaffold. Pyridine analogues were synthesized by the routes shown in Schemes 2 and 3. These involved nucleophilic addition of cyclohexylamine to the halopyrimidine, followed by a palladium-catalyzed coupling reaction to install the 3-pyridinyl3-ylaniline functional group. Schemes 4-7 depict the routes used to synthesize analogues in which the amine linkers at the 2-and/ or 4-positions of the aminopyrimidine were replaced with $\mathrm{N}$ methyl or oxygen. $\mathrm{N}$-Methylcyclohexane was installed at the 2position by treating with triethylamine (Scheme 4), whereas cyclohexanol was incorporated by reacting with 2,4-dichloropyrimidine in the presence of sodium hydride (Scheme 5). Substituents at the 4-position were installed using catalytic $\mathrm{HCl}$ and by refluxing in ethanol (EtOH) (Schemes 4-7). Overall, the routes proceeded with sufficient yield (3-85\% yield), producing all targeted analogues for characterization, although, undoubtedly, further optimization could be beneficial for individual analogues. All compounds used for subsequent testing were purified to greater than $95 \%$ purity, as confirmed by the combination of liquid chromatography-mass spectrometry (LC-MS) and ${ }^{1} \mathrm{H}$ NMR studies.

SAR. We utilized the FLT3 ${ }^{\mathrm{WT}}$ enzyme assay to determine the potencies of all analogues of $\mathbf{1}$ made during this study (Table S2). In parallel, we tested the effects on the proliferation of MV4-11 cells (Table S2). We also measured the effects of these compounds on the proliferation of untransformed BJ fibroblasts to evaluate the dependency of cellular effects upon the presence of a driving FLT3 mutation (Table S3). Because cellular assays include fetal bovine serum within the media, there is a potential that the $\mathrm{EC}_{50}$ values could differ from the actual values due to plasma protein binding. ${ }^{61}$ The solubility and permeability as well as the potential susceptibility to P-glycoprotein (PGP) efflux were assessed for selected compounds (Tables S4 and S5) to aid in interpreting the data and planning for structural modifications.

The initial focus of the project was defining the minimal pharmacophore for the inhibitors. Replacement of the nitrogen at the 1-position with a carbon atom (9) did not have a large impact on potency for kinase inhibition but completely ablated effects upon proliferation of MV4-11 cells (Table 2). Removal of the nitrogen at the 3-position (13) reduced the potency of kinase inhibition compared to that of $\mathbf{1}$, while adding weak antiproliferative effects on untransformed BJ cells (Table S3). 
Table 1. $K_{\mathrm{d}}$ Values for 1

\begin{tabular}{|c|c|c|c|}
\hline kinase & $K_{\mathrm{d}}(\mathrm{nM})^{a}$ & kinase & $K_{\mathrm{d}}(\mathrm{nM})^{a}$ \\
\hline ABL1 (H396P)-phosphorylated & 2100 & LCK & 2900 \\
\hline AURKA & 1600 & MERTK & 2800 \\
\hline AURKC & $>10000$ & MINK & 2500 \\
\hline AXL & 2100 & NEK10 & 410 \\
\hline BLK & 3900 & PDGFRB & 120 \\
\hline CLK1 & 430 & PIK4CB & 370 \\
\hline CLK4 & 690 & PIP5K1C & 610 \\
\hline CSF1R & 590 & PRKCQ & 1600 \\
\hline CSF1R-autoinhibited & 1500 & PRKD3 & 1700 \\
\hline EPHB6 & 560 & RET (M918T) & 1200 \\
\hline FLT3 & 61 & RET (V804M) & 1400 \\
\hline $\mathrm{HCK}$ & 2100 & RSK1 (Kin.Dom.1-N-terminal) & 4400 \\
\hline IRAK1 & 1500 & SRC & 1100 \\
\hline IRAK3 & 1400 & TRKA & 1100 \\
\hline JAK1 (JH2 domain-pseudokinase) & 290 & TYK2 (JH1 domain-catalytic) & 350 \\
\hline JAK2 (JH1 domain-catalytic) & 260 & TYK2 (JH2 domain-pseudokinase) & 740 \\
\hline JAK3 (JH1 domain-catalytic) & 1300 & TYRO3 & 2700 \\
\hline KIT & 1500 & & \\
\hline
\end{tabular}

Scheme 1. General Route for the Synthesis of 2,4Aminopyrimidine Analogues ${ }^{a}$

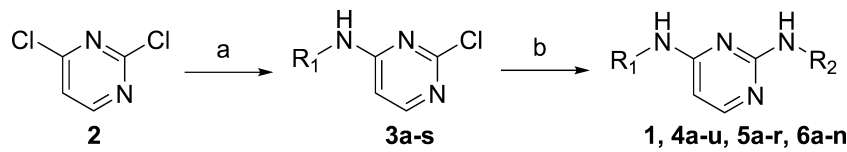

${ }^{a}$ Reagents and conditions: (a) $\mathrm{NH}_{2}-\mathrm{R}_{1}$, dichloromethane (DCM) or methanol $(\mathrm{MeOH})$ or $\mathrm{NH}_{2}-\mathrm{R}_{1}$, triethylamine, DCM, rt, 5-16 h, 1$60 \%$; (b) $\mathrm{NH}_{2}-\mathrm{R}_{2}$, catalytic $\mathrm{HCl}, \mathrm{EtOH}$ or $\mathrm{MeOH}$, reflux, 1-22 h, 3$85 \%$.

Compound 14, in which the location of the ring nitrogen had shifted, exhibited good enzymatic inhibitory potency but lacked MV4-11 cellular activity and also was weakly active against BJ fibroblasts (Table S3). Therefore, the pyrimidine core was necessary for optimal activity.

We examined the effects of replacing the linker atoms on both FLT3 inhibition and MV4-11 proliferation using both $N$-methyl and ether replacements (Table 3 ). Although replacement of the 2 -amine with $N$-methyl abrogated activity against FLT3 $(21,17)$, 17 possessed modest cellular potency, which might be due to metabolic removal of the methyl groups (unexplored). Replacement of the amine linkers with ethers at either the 4position (19) or both the 2- and 4-position (20) did not greatly impact the enzymatic activity, whereas replacement of only the 2nitrogen with an ether linkage did reduce the enzymatic activity nearly 40 -fold compared to that of $\mathbf{1}$ (22). However, compounds 19, 22, and 20 were all devoid of effects on MV4-11 cell proliferation. Although the lack of enzymatic inhibition exhibited by $\mathrm{N}$-methylated compounds $\mathbf{1 7}$ and $\mathbf{2 1}$ could suggest that a key hydrogen-bonding interaction may be made with FLT3 through the amine at the 2-position, compound 20, which contains an ether at this location, also demonstrated potent FLT3 inhibition. Therefore, it is not clear what particular interactions occur between these compounds and the kinase. Overall, however, the inclusion of amine linkers at the 2- and 4-position provided the best combination of enzymatic and cellular activity. On the basis of these results, we focused on the 2,4-aminopyrimidine scaffold for further optimization.

Next, we explored the replacement of the pendent hydrophobic groups surrounding the aminopyrimidine scaffold. First, the cyclohexyl substituent at the 4-position was fixed, while the substituent at the 2-position was systematically varied (Table 4). Paring the structure down by removing the pyridine ring (4a) caused a reduction in FLT3 inhibitory activity and ablated activity in MV4-11 cells; therefore, this functional group may improve binding to FLT3 through a potential a H-bonding interaction. Reductions in FLT3 inhibition and MV4-11 cellular activity were also observed with compounds $4 \mathbf{c}-\mathbf{f}$, which also did not contain the pyridine ring in 1 . Replacement of the 3-(pyridin3-yl)aniline moiety with either 2- or 3-substituted biphenyl (4b, $\mathbf{4 g}$ ) abolished all activity. Incorporation of a 3'-methoxybiphenyl (4i) or methyl ester $(\mathbf{4} \mathbf{j})$ led to a reduction in enzymatic and MV4-11 cellular activities compared to those of 1 , and these compounds also gained weak activity against BJ cells (Table S3). Shifting the location of the ring nitrogen $(4 \mathbf{h})$ reduced the activity compared to that of $\mathbf{1}$. However, replacement of the 3 -

\section{Scheme 2. Synthesis of Compound $9^{a}$}

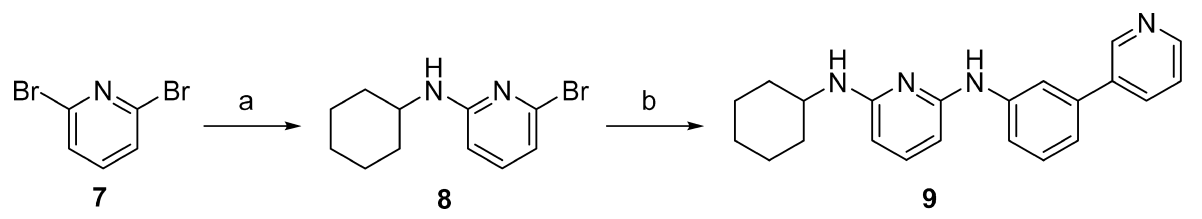

${ }^{a}$ Reagents and conditions: (a) aminocyclohexane, triethylamine, $\mathrm{N}$-methyl-2-pyrrolidone (NMP), $100{ }^{\circ} \mathrm{C}, 21 \mathrm{~h}, 10 \%$; (b) 3-pyridinyl-3-ylaniline, $\mathrm{Pd}_{2}(\mathrm{dba})_{3}, \mathrm{R}$-BINAP, sodium tert-butoxide, toluene, $80{ }^{\circ} \mathrm{C}, 16 \mathrm{~h}, 56 \%$. 


\section{Scheme 3. Synthesis of Compounds 13 and $14^{a}$}

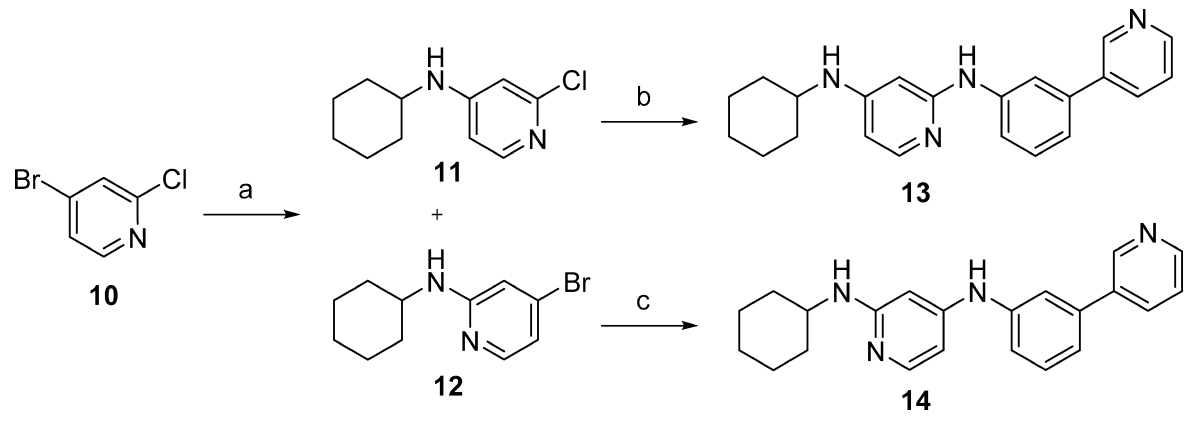

${ }^{a}$ Reagents and conditions: (a) aminocyclohexane, triethylamine, NMP, $80{ }^{\circ} \mathrm{C}, 16 \mathrm{~h}, 15 \% 11,2 \% 12$; (b) 3-pyridinyl-3-ylaniline, $P d_{2}\left(\mathrm{dba}_{3}\right)_{3}, R-$ BINAP, sodium tert-butoxide, dimethylformamide (DMF), $140{ }^{\circ} \mathrm{C}, 35 \mathrm{~mW}, 1 \mathrm{~h}, 24 \%$; (c) 3-pyridinyl-3-ylaniline, $\mathrm{Pd}_{2}\left(\mathrm{dba}_{3}, R\right.$-BINAP, sodium tertbutoxide, toluene, $80{ }^{\circ} \mathrm{C}, 16 \mathrm{~h}, 9 \%$.

\section{Scheme 4. Synthesis of Compounds 16 and $17^{a}$}

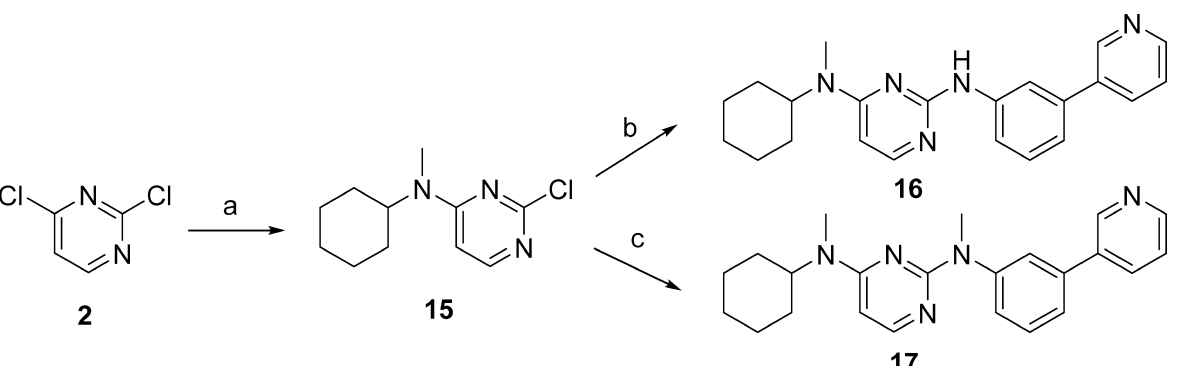

${ }^{a}$ Reagents and conditions: (a) N-methylaminocyclohexane, triethylamine, ethanol, reflux, $5 \mathrm{~h}, 59 \%$; (b) 3-pyridinyl-3-ylaniline, catalytic $\mathrm{HCl}$, ethanol, reflux, $3 \mathrm{~h}, 25 \%$; (c) $\mathrm{N}$-methyl-3-(pyridin-3-yl)aniline, catalytic $\mathrm{HCl}$, ethanol, reflux, $2 \mathrm{~h}, 27 \%$.

\section{Scheme 5. Synthesis of Compounds 19 and $20^{a}$}

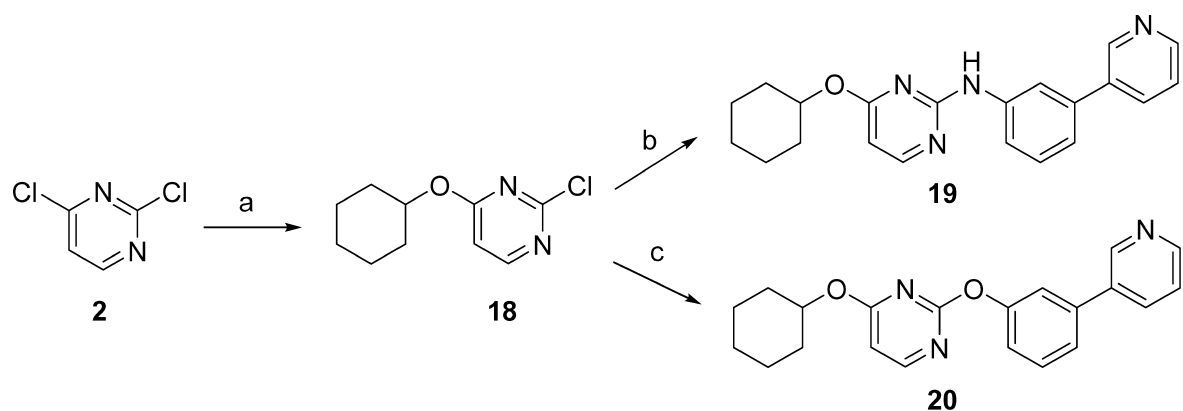

${ }^{a}$ Reagents and conditions: (a) cyclohexanol, sodium hydride, DMF, $0{ }^{\circ} \mathrm{C}$ to $\mathrm{rt}, 16 \mathrm{~h}, 3 \%$; (b) 3-pyridinyl-3-ylaniline, catalytic $\mathrm{HCl}$, ethanol, reflux, 3 h, 9\%; (c) 3-(pyridin-3-yl)phenol, catalytic $\mathrm{HCl}$, ethanol, reflux, 16 h, 52\%.

Scheme 6. Synthesis of Compound $21^{a}$

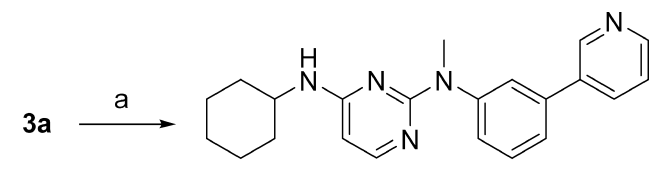

21

${ }^{a}$ Reagents and conditions: $N$-methyl-3-(pyridin-3-yl)aniline, catalytic $\mathrm{HCl}$, ethanol, reflux, $2 \mathrm{~h}, 6 \%$.

pyridinyl group with other nitrogen-containing heterocycles was tolerated by FLT3 $(4 \mathbf{k}-\mathbf{n})$, further suggesting that a nitrogen atom in this position may provide critical interactions with FLT3. Compounds 4o, $4 \mathrm{p}$, and $4 \mathrm{r}$, which also lacked a pyridinyl nitrogen, showed diminished FLT3 inhibition compared to that by $\mathbf{1}$ as well. Although compound $\mathbf{4 q}$ contained the desired
Scheme 7. Synthesis of Compound $22^{a}$<smiles>c1cncc(-c2cccc(Oc3nccc(NC4CCCCC4)n3)c2)c1</smiles>

22

${ }^{a}$ Reagents and conditions: 3-(pyridin-3-yl)phenol, catalytic $\mathrm{HCl}$, ethanol, reflux, $16 \mathrm{~h}, 81 \%$.

pyridine ring, altered molecular conformations may have led to this compound's lack of activity. Compound $4 s$ also exhibited good activity, suggesting that the carboxamide moiety may be in a beneficial location for hydrogen-bonding interactions with FLT3. Inclusion of a pyridine ring at the para position (4t) provided modest FLT3 activity but good MV4-11 cellular 
Table 2. SAR of the Aminopyrimidine Ring for Compounds 9, 13, 14, and Quizartinib

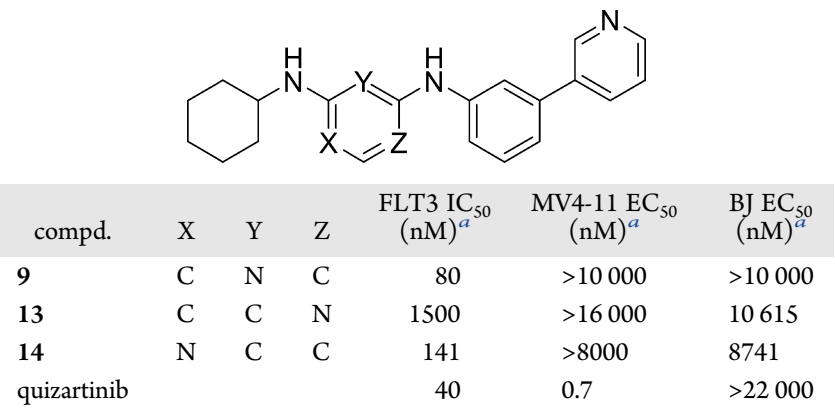

${ }^{a} \mathrm{IC}_{50}$ and $\mathrm{EC}_{50}$ values are reported as the mean of triplicates. Values for CI 95 are included in Table S3.

Table 3. SAR Analysis of Modifications to the Linkers for Compounds 16, 17, and 19-22

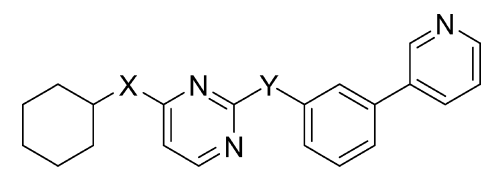

\begin{tabular}{cllcc} 
compd. & \multicolumn{1}{c}{ X } & \multicolumn{1}{c}{$\mathrm{Y}$} & ${\text { FLT3 } \mathrm{IC}_{50}(\mathrm{nM})^{a}}$ & $\mathrm{MV}^{-11 \mathrm{EC}_{50}(\mathrm{nM})^{a}}$ \\
$\mathbf{1 6}$ & $\mathrm{N}-\mathrm{Me}$ & $\mathrm{NH}$ & 82 & $>8000$ \\
$\mathbf{2 1}$ & $\mathrm{NH}$ & $\mathrm{N}-\mathrm{Me}$ & $>11000$ & $>4000$ \\
$\mathbf{1 7}$ & $\mathrm{N}-\mathrm{Me}$ & $\mathrm{N}-\mathrm{Me}$ & $>15000$ & 39 \\
$\mathbf{1 9}$ & $\mathrm{O}$ & $\mathrm{NH}$ & 187 & $>6000$ \\
$\mathbf{2 2}$ & $\mathrm{NH}$ & $\mathrm{O}$ & 1260 & $>14000$ \\
$\mathbf{2 0}$ & $\mathrm{O}$ & $\mathrm{O}$ & 49 & $>13000$
\end{tabular}

${ }^{a} \mathrm{IC}_{50}$ and $\mathrm{EC}_{50}$ values are reported as the mean of triplicates. Values for CI 95 are included in Table S3.

activity. Although compound $\mathbf{4} \mathbf{u}$ was inactive against FLT3, an effect on MV4-11 cell proliferation was observed. The potential off-target kinase activity of this compound was not investigated, but interactions with other kinases may contribute to the moderate cellular activity of compounds $4 \mathbf{t}$ and $4 \mathbf{u}$. Taken together, these results point to a very strong preference for particular steric and electronic arrangements on this portion of the molecule, with the original 3-pyridinylphenyl presenting a good balance of properties.

In parallel, we synthesized a series of compounds keeping the 3-pyridinyl-3-ylaniline moiety constant, while varying the aliphatic substituents at the 4-position. Short linear, branched, and cyclic aliphatic groups were evaluated (Table 5). Although reduced enzymatic inhibition and a loss of MV4-11 cellular activity was observed when replacing the cyclohexyl group with a methyl (5a), good potency was obtained with either an ethyl (5b) or propyl (5c) group and moderate potency was obtained with a butyl (5d) group. Branched alkyl chains, such as isopropyl (5e), sec-butyl (5j, 5k), and tert-butyl (5f), provided improvements in the FLT3 inhibitory activity and approximately 10-fold improvements in MV4-11 growth inhibition relative to those of 1. Inclusion of ether $(\mathbf{5 g})$, ester $(\mathbf{5 h})$, or carboxylic acid $(\mathbf{5 i})$ functionalities within the branched aliphatic substituents greatly reduced cellular activity. Although $\mathbf{5 g}$ had good solubility and permeability, the lower stability of the methoxy group in the cellular environment may have impacted its cellular activity (not explored). Some compounds with aliphatic rings, such as $\mathbf{5 1}$ and $\mathbf{5 m}$, demonstrated good enzymatic and cellular activities. Although both the cyclopropyl and cyclobutyl (5n) groups afforded good potency against the FLT3 enzyme, poor solubility (Table S4) may have hampered the cellular activity of the latter. Despite its low solubility, compound 5o exhibited good cellular activity. Therefore, additional properties beyond solubility could potentially have subtle effects on activity. Although $\mathbf{5 q}$ was approximately 4-fold more potent than 1 against FLT3, the potency against MV4-11 cells was not improved due to poor cellular permeability (Table S4). Inclusion of a methoxy group (5p) was not beneficial. Replacing the amine functionality in the aliphatic ring of $\mathbf{5 q}$ with an ether $(\mathbf{5 r})$ provided only moderate enzymatic and cellular activities.

Because of its improved activity against FLT3 and MV4-11 cells compared to that of 1 , we fixed the 4-position as the isopropyl group and varied the 2-position of the compound to determine whether the activity trends observed would be similar to those in the cyclohexyl series, as previously shown in Table 4. Similar to that in the cyclohexyl series, inclusion of a methoxy group (6e) or replacement of the pyridine functionality with a 2-, 3-, or 4-biphenyl $(6 \mathrm{a}, 6 \mathrm{c}, \mathbf{6 m})$ caused decreases in both FLT3 inhibitory activity and cellular potency (Table 6). Inclusion of a tert-butyl group in place of the pyridine ring $(6 \mathbf{b})$ abrogated activity as well. Shifting the nitrogen from the 3-position to the 2position (6d) or the 4-position (6l) also reduced potency, paralleling our prior observations and suggesting that a nitrogen at the 3-position may act as an $\mathrm{H}$-bond acceptor to make an important binding interaction. Incorporation of a methyl ester (6f) led to reduced activity. The potency was comparable to that of 1 when the 3-pyridinyl group was replaced with a pyrazole (6i), and inhibition of MV4-11 cell proliferation improved when an imidazole $(6 \mathbf{j})$ or pyrazine $(\mathbf{6 k})$ replaced the pyridine. Compounds containing oxazole $(\mathbf{6 g})$ or thiazole $(6 \mathrm{~h})$ moieties, however, provided less potent FLT3 inhibition and MV4-11 cellular activity. Similar to the effect observed with $4 \mathbf{s}$, the carboxamide-functionalized compound $6 \mathrm{n}$ also exhibited moderate enzymatic inhibition and activity against MV4-11 cells.

On the basis of the balance of activity, we selected 5e for further profiling. The compound retained its FLT3 potency (3.6 $\mathrm{nM}$ ) and selectivity on the basis of profiling the $K_{\mathrm{d}}$ values for a subset of kinases inhibited by 1 (Table 7 ). Despite potent binding to PDGFRB (28 $\mathrm{nM})$ and the JAK1 JH2 domain (28 $\mathrm{nM})$, 5e was still approximately 8 -fold more potent against FLT3. Therefore, modifications explored during the SAR studies did not appear to significantly affect kinase selectivity compared to that of the initial hit compound.

In Vitro Effects against FLT3 Mutants and MOLM13 Cells. Because of the demonstrated activity of this series of compounds in MV4-11 cells, we evaluated a subset of compounds to determine whether they inhibited the proliferation of other FLT3 mutant AML cells. The initial hit compound 1 and $5 \mathbf{e}, 6 \mathbf{k}$, and a structurally related inactive compound $\mathbf{6 a}$ were tested for their ability to inhibit the proliferation of MOLM13 cells, which also contain the FLT3-ITD mutation, and a sorafenib-resistant progeny of MOLM13 that has acquired an additional tyrosine kinase domain (TKD) mutation, Asp835. ${ }^{50}$ As shown in Figure 2, 6k was the most potent at inhibiting MOLM13 cell viability $\left(\mathrm{IC}_{50}=45 \mathrm{nM}\right.$; CI 95, 38-55 nM), followed by 5 e $\left(\mathrm{IC}_{50}=136 \mathrm{nM}\right.$; CI 95, 112-164 nM). The relative sensitivity of MOLM13 cells to $5 \mathbf{e}$ and $\mathbf{6 k}$ was correlated to the loss of FLT3-ITD phosphorylation and downstream STAT5 signaling, with FLT3 $\mathrm{IC}_{50}$ values estimated to be within 50-100 nM in these experiments (Figure 3). In addition, all three tested compounds $(\mathbf{1}, \mathbf{5 e}$, and $\mathbf{6 k})$ possessed similar growth inhibitory potencies against both parental MOLM $13^{\text {FLT3-ITD }}$ and 
Table 4. SAR of the Substituents at the Aminopyrimidine 2-Position for Compounds 1 and $4 a-u^{a}$

\begin{tabular}{|c|c|c|c|c|c|c|c|}
\hline Compd. & $\mathrm{R}_{1}$ & $\begin{array}{c}\text { FLT3 } \\
\mathrm{IC}_{50} \\
(\mathrm{nM})^{a}\end{array}$ & $\begin{array}{l}\text { MV4- } \\
11 \mathrm{EC}_{50} \\
(\mathrm{nM})^{a}\end{array}$ & Compd. & $\mathrm{R}_{1}$ & $\begin{array}{c}\text { FLT3 } \\
\text { IC }_{50} \\
(\mathrm{nM})^{a}\end{array}$ & $\begin{array}{c}\text { MV4- } \\
11 \\
\mathrm{EC}_{50} \\
(\mathrm{nM})^{a} \\
\end{array}$ \\
\hline 1 & & 32 & 320 & $4 k$ & & 336 & 511 \\
\hline $4 a$ & & 1890 & $>7000$ & 41 & & 96 & $>14000$ \\
\hline $4 b$ & & $>13000$ & $>5000$ & $4 m$ & & 16 & 58 \\
\hline $4 c$ & & 2590 & $>15000$ & $4 n$ & & 20 & 53 \\
\hline $4 d$ & & 379 & 5050 & 40 & & 722 & 369 \\
\hline $4 e$ & & $>19000$ & $>7000$ & $4 p$ & & 3260 & 3570 \\
\hline $4 f$ & & 978 & 5230 & $4 q$ & & $>23000$ & $>8000$ \\
\hline $4 g$ & & $>12000$ & $>4000$ & $4 r$ & & 3300 & $>3000$ \\
\hline $4 h$ & & 1710 & 3230 & $4 s$ & & 14 & 113 \\
\hline $4 i$ & & 24500 & $>15000$ & $4 t$ & & 1880 & 41 \\
\hline $4 j$ & & 13300 & 7590 & $4 u$ & & $>31000$ & 1260 \\
\hline
\end{tabular}

${ }^{a} \mathrm{IC}_{50}$ and $\mathrm{EC}_{50}$ values are reported as the mean of triplicates. Values for CI 95 are included in Table S3.

progeny MOLM13 ${ }^{\text {FLT3-ITD/D835Y }}$ cells. We also determined the $K_{\mathrm{d}}$ values for compounds 1, 5e, and 6a against several FLT3 mutants (Table 8). ${ }^{62}$ Compound 5e bound more tightly to all FLT3 variants tested compared with $\mathbf{1}$, whereas $\mathbf{6 a}$ did not bind to any FLT3 variant at any tested concentration. Therefore, there was very good correlation between the improved potency of $\mathbf{5 e}$ against MOLM13 cells and improved binding to FLT3 variants.
Interestingly, the fold-difference in the affinities of $\mathbf{5 e}$ and $\mathbf{1}$ was very similar between the FLT3 and Asp835 mutants, which is mirrored by similar potencies in parental and kinase inhibitorresistant MOLM13 cells. This is a desirable profile for a new FLT3 inhibitor scaffold. Previously studied type I kinase inhibitors have also had similar to better affinities for FLT3, FLT3-ITD, or activating TKD mutations, suggesting that these 
Table 5. SAR of the Substituents at the Aminopyrimidine 4-Position for Compounds 5a-ra,b

\begin{tabular}{|c|c|c|c|c|c|c|c|}
\hline Compd. & $\mathrm{R}_{1}$ & $\begin{array}{c}\text { FLT3 } \\
\mathrm{IC}_{50} \\
(\mathrm{nM})^{a} \\
\end{array}$ & $\begin{array}{c}\mathrm{MV}-11 \\
\mathrm{EC}_{50} \\
(\mathrm{nM})^{a}\end{array}$ & Compd. & $\mathrm{R}_{1}$ & $\begin{array}{c}\text { FLT3 } \\
\mathrm{IC}_{50} \\
(\mathrm{nM})^{a} \\
\end{array}$ & $\begin{array}{c}\mathrm{MV}^{4-11} \\
\mathrm{EC}_{50} \\
(\mathrm{nM})^{a}\end{array}$ \\
\hline $5 a$ & $\mathrm{Me}^{-}$ & 240 & $>20000$ & $5 \mathbf{j}$ & & 8 & 65 \\
\hline $5 b$ & $\mathrm{Et}^{\prime}$ & 47.5 & 35 & $5 \mathbf{k}$ & & $<6^{b}$ & 25 \\
\hline $5 c$ & & $<6^{b}$ & 138 & 51 & & $<6^{b}$ & 141 \\
\hline $5 d$ & & 123 & 340 & $5 \mathrm{~m}$ & & $<6^{b}$ & 31 \\
\hline $5 e$ & & $<6^{b}$ & 25 & $5 n$ & & $<6^{b}$ & $>17000$ \\
\hline $5 f$ & $\mathrm{Bu}$ & 13 & 25 & 50 & & 13 & 58 \\
\hline $5 g$ & & $<6^{b}$ & $>23000$ & $5 p$ & & $>34000$ & $>12000$ \\
\hline $5 \mathrm{~h}$ & & $>22000$ & $>8000$ & $5 q$ & & 8 & 654 \\
\hline $5 \mathbf{i}$ & & $>23000$ & $>8000$ & $5 r$ & & 187 & 1877 \\
\hline
\end{tabular}

${ }^{a} \mathrm{IC}_{50}$ and $\mathrm{EC}_{50}$ values are reported as the mean of triplicates. Values for CI 95 are included in Table S3. ${ }^{b}$ On the basis of assay conditions, $\mathrm{IC}_{50}$ values below $6 \mathrm{nM}$ cannot be accurately measured.

compounds may have the properties of a type I inhibitor, which binds in the ATP-binding pocket of the kinase. ${ }^{63}$ Additionally, when selected compounds were evaluated in the Z'LYTE FLT3 kinase inhibition assay under standard conditions but using either a lower $(100 \mu \mathrm{M})$ or higher $(2.5 \mathrm{mM})$ concentration of ATP, shifts in the FLT3 IC $_{50}$ values of these compounds were observed. For instance, the $\mathrm{IC}_{50}$ of the initial hit, $\mathbf{1}$, was reduced to $<6 \mathrm{nM}$ in the presence of $100 \mu \mathrm{M}$ ATP and increased to 116 $\mathrm{nM}(\mathrm{CI} 95,70-193 \mathrm{nM})$ when the assay was conducted at a 2.5 mM ATP concentration. Similar to other type I kinase inhibitors, these compounds may make crucial binding interactions within the ATP site and therefore may not be sensitive to the conformation of the DFG motif.

In Vitro ADME Studies. We evaluated the in vitro pharmacological characteristics of $\mathbf{1}, \mathbf{5 e}$, and $\mathbf{6 k}$ to assess their potential for use in in vivo studies. All three analogues had excellent passive permeabilities $\left(1880 \pm 170 \times 10^{-6}, 2000 \pm 190\right.$ $\times 10^{-6}$, and $1870 \pm 60 \times 10^{-6} \mathrm{~cm} / \mathrm{s}$, respectively), as measured in the PAMPA assay. Neither 1 nor 5e showed susceptibility to PGP efflux (Table S5) in cellular permeability assays. Compound 6k was not evaluated in this assay. Although the solubility of $\mathbf{1}$ in PBS was poor $(0.4 \pm 0.9 \mu \mathrm{M})$, that of 5e was reasonable $(26.4 \pm$ $17.0 \mu \mathrm{M})$. Finally, the stability of these three compounds in liver microsomes from four species was lower than desirable (Table 9). However, the plasma stability of $\mathbf{5 e}$ and $\mathbf{6 k}$ was excellent ( $>48$ h). Overall, these studies would predict reasonable oral absorption for 5e but rapid clearance (CL) for all three, and it would be expected that first-pass metabolism effects could cause low bioavailability.

In Vivo Pharmacokinetics. On the basis of the results from the microsomal stability studies of $\mathbf{1}, \mathbf{5 e}$, and $\mathbf{6 k}$, we selected $\mathbf{5 e}$ to evaluate in vivo pharmacokinetics at three doses by intraperitoneal (i.p.) injection. A greater than proportional dosedependent increase in $C_{\max }$ (Table 10) was observed (327, 927, and $1625 \mathrm{nM}$ for 3,5 , and $10 \mathrm{mg} / \mathrm{kg}$ respectively) and the halflife of the compound ranged from 0.23 to $1 \mathrm{~h}$, paralleling our observations in mouse microsome models $(0.2-1 \mathrm{~h}$ at $0.8-20$ $\mu \mathrm{M}$ ). The area under the concentration-time curve (AUC) within $24 \mathrm{~h}$ also displayed a dose-dependent trend. CL, however, was high at all doses tested. Therefore, because of the undesirable pharmacokinetic profile of 5e, we did not pursue efficacy modeling using a human AML xenograft model with this compound because its pharmacokinetic properties could potentially confound the results of such a study.

\section{CONCLUSIONS}

Starting with hit compound 1, we developed a SAR concerning inhibition of FLT3 enzymatic activity and MV4-11 cellular proliferation. Overall, the SAR for enzymatic inhibition and inhibition of cellular proliferation correlated well. We found that the pyrimidine nitrogens and nitrogen linker atoms were important for MV4-11 cellular activity. Inclusion of a 3pyridinylphenyl group at the 2-position and a small aliphatic substituent at the 4-position provided a balance of potency, solubility, and permeability (Figure 4). Additionally, the pan- 
Table 6. Additional SAR at the Aminopyrimidine 2-Position for Compounds $6 a-n^{a, b}$

\begin{tabular}{|c|c|c|c|c|c|c|c|}
\hline $\begin{array}{c}\text { Compd } \\
.\end{array}$ & $\mathrm{R}_{1}$ & $\begin{array}{c}\text { FLT3 } \\
\mathrm{IC}_{50} \\
(\mathrm{nM})^{a}\end{array}$ & $\begin{array}{c}\text { MV4- } \\
11 \\
\mathrm{EC}_{50} \\
(\mathrm{nM})^{a}\end{array}$ & Compd. & $\mathrm{R}_{1}$ & $\begin{array}{c}\text { FLT3 } \\
\text { IC }_{50} \\
(\mathrm{nM})^{a}\end{array}$ & $\begin{array}{c}\text { MV4- } \\
11 \\
\mathrm{EC}_{50} \\
(\mathrm{nM})^{a}\end{array}$ \\
\hline $6 a$ & & $>24000$ & $>16000$ & $6 h$ & & 475 & 9460 \\
\hline $6 b$ & & 17200 & $>13000$ & $6 \mathbf{i}$ & & 42 & 459 \\
\hline $6 c$ & & 11600 & 3980 & $\mathbf{6 j}$ & & $<6^{b}$ & 4 \\
\hline 6d & & 2880 & 4820 & $6 \mathrm{k}$ & & 11 & 5 \\
\hline $6 e$ & & 6970 & $>16000$ & 61 & & 284 & $>15000$ \\
\hline $6 f$ & & 4440 & 4890 & $6 \mathrm{~m}$ & & 15000 & 11900 \\
\hline $6 \mathrm{~g}$ & & 918 & 1060 & $6 n$ & & 213 & 2530 \\
\hline
\end{tabular}

${ }^{a} \mathrm{IC}_{50}$ and $\mathrm{EC}_{50}$ values are reported as the mean of triplicates. Values for $\mathrm{CI} 95$ are included in Table S3. ${ }^{b}$ On the basis of assay conditions, $\mathrm{IC}_{50}$ values below $6 \mathrm{nM}$ cannot be accurately measured.

Table 7. $K_{\mathrm{d}}$ Values for $5 \mathrm{e}$

\begin{tabular}{|c|c|}
\hline kinase & $K_{\mathrm{d}}(\mathrm{nM})^{a}$ \\
\hline CLK1 & 260 \\
\hline CLK4 & 1100 \\
\hline CSF1R & 460 \\
\hline EPHB6 & 440 \\
\hline FLT3 & 3.6 \\
\hline JAK1 (JH2 domain-pseudokinase) & 28 \\
\hline JAK2 (JH1 domain-catalytic) & 480 \\
\hline NEK10 & 2000 \\
\hline PDGFRB & 28 \\
\hline PIK4CB & 330 \\
\hline PIP5K1C & 1300 \\
\hline TYK2 (JH1 domain-catalytic) & 740 \\
\hline TYK2 (JH2 domain-pseudokinase) & 210 \\
\hline
\end{tabular}

activity against FLT3 mutations and the relatively high selectivity for FLT3 were both generally maintained during the SAR studies, suggesting that the scaffold might provide good lead molecules for further development. From these studies, we developed 5e, which not only had improved potency against FLT3 and MV4-11 cells but also demonstrated activity in MOLM13 cells possessing FLT3-ITD and FLT3 inhibitor-resistant TKD mutations. Compound 5e possessed acceptable in vitro ADME properties and was selected for additional preclinical studies. However, because of its low metabolic stability and high CL in pharmacokinetic studies, we determined that these properties need to be improved before further preclinical studies are undertaken. Efforts toward this goal are on-going.

\section{EXPERIMENTAL SECTION}

Chemistry. All chemical reagents were purchased from commercial suppliers (Acros, Combi-Blocks, Enamine, Oakwood, Sigma Aldrich, Strem) and were used without further purification. Solvents were dried using a column-exchange system $^{64}$ Thin-layer chromatography (TLC) was performed using Merck Millipore silica gel 60G $\mathrm{F}_{254}$ glass plates and visualized using a $254 \mathrm{~nm}$ UV lamp for detection. Microwave 

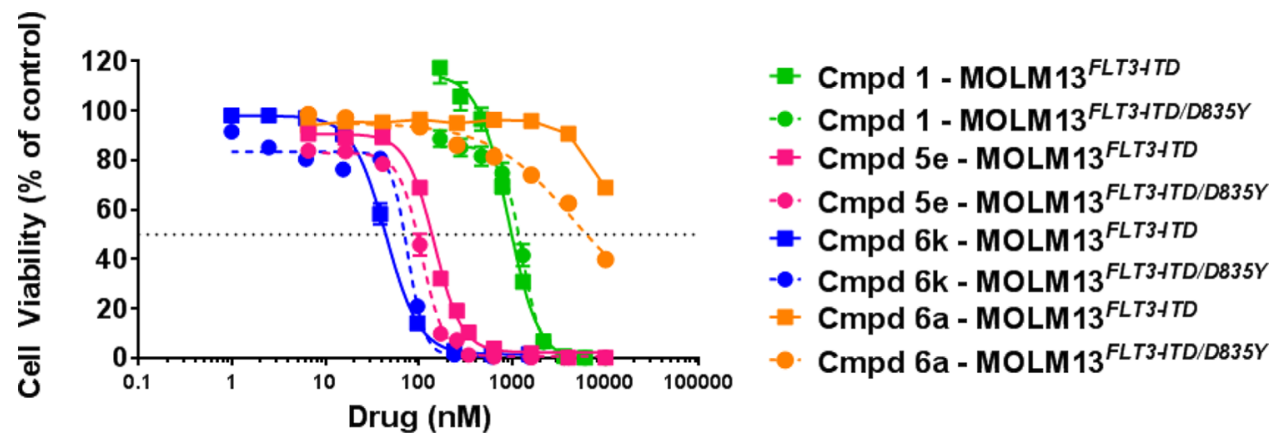

Figure 2. Relative inhibition of the proliferation of MOLM13 cells carrying either FLT3-ITD or FLT3-ITD with an additional mutation of D835Y by compounds $\mathbf{1}, \mathbf{5 e}, \mathbf{6 k}$, and $\mathbf{6 a}$, as evaluated in the MTT assay. Data are expressed as the mean of cell viability measurements \pm SE of three experiments, with six replicates each $(n=18)$, after treatment with drug for $72 \mathrm{~h}$. $\left(\mathrm{IC}_{50}\right.$ and $\mathrm{CI} 95$ in $\mathrm{nM}$ : MOLM13 ${ }^{\text {FLT3-ITD }} \mathbf{1}=620$, 461-833;

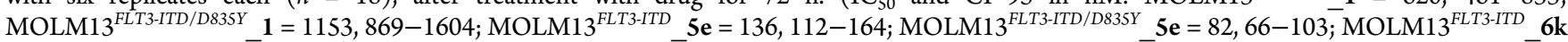

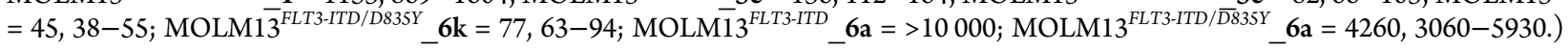

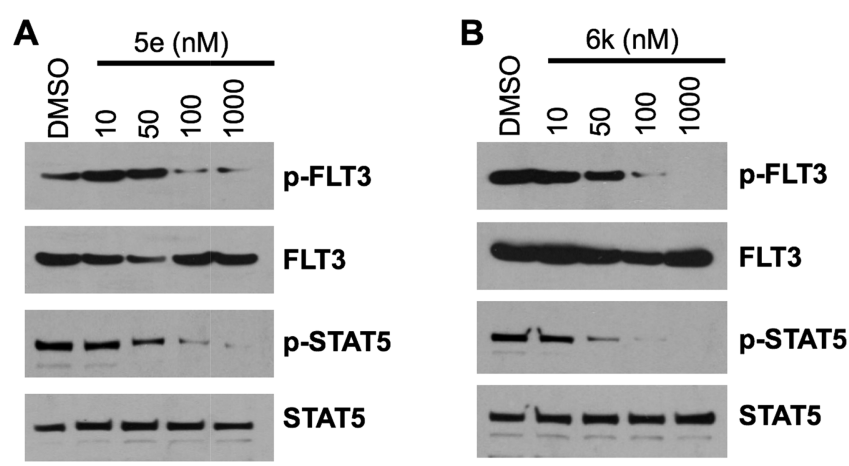

Figure 3. Inhibition of FLT3 signaling by compounds $5 \mathbf{e}$ and $\mathbf{6 k}$ MOLM13 $3^{\text {FLT3-ITD }}$ cells were treated for $1 \mathrm{~h}$ with dimethyl sulfoxide (DMSO) or increasing concentrations of (A) compound 5e or (B) compound $6 \mathbf{k}$ and lysed. Western blot analysis was performed on the FLT3 immunoprecipitation eluent or the whole-cell lysate using the indicated antibodies.

Table 8. $K_{d}$ Values for $1,5 e$, and 6a Bound to Mutant FLT3 Kinases

\begin{tabular}{lccc}
\multicolumn{1}{c}{ kinase } & $\mathbf{1} K_{\mathrm{d}}(\mathrm{nM})^{a}$ & $\mathbf{5 e ~} K_{\mathrm{d}}(\mathrm{nM})^{a}$ & $\mathbf{6 a} K_{\mathrm{d}}(\mathrm{nM})^{a}$ \\
FLT3 & 61 & 3.6 & $>10000$ \\
FLT3 (D835H) & 22 & 1.2 & $>10000$ \\
FLT3 (D835V) & 7.7 & 0.8 & $>10000$ \\
FLT3 (D835Y) & 13 & 0.59 & $>10000$ \\
FLT3 (ITD) & 31 & 1.4 & $>10000$ \\
FLT3 (ITD, D835V) & 7.2 & 0.36 & $>10000$ \\
FLT3 (ITD, F691L) & 270 & 11 & $>10000$ \\
FLT3 (K663Q) & 110 & 13 & $>10000$ \\
FLT3 (N841I) & 48 & 11 & $>10000$ \\
FLT3 (R834Q) & 680 & 82 & $>10000$ \\
FLT3-autoinhibited & 1100 & 78 & $>10000$
\end{tabular}

${ }^{a} K_{\mathrm{d}}$ values are reported as the mean of two experiments.

experiments were carried out using a Biotage Initiator system. Automated flash chromatography was performed using the Biotage SP1 flash column system with silica gel SNAP columns or C18 SNAP columns for reversed-phase purifications. ${ }^{65} R_{f}$ values are quoted for the eluent system stated. Evaporation was carried out using a Büchi Rotovapor. NMR spectra were recorded on either a Bruker $400 \mathrm{MHz}$ or Bruker $500 \mathrm{MHz}$ spectrometer in the solvents indicated, and the spectra were processed using MestReNova (8.1) referenced to the solvent peak. Melting points were obtained using a Büchi-545. Optical rotations were recorded using a Jasco P-1010 polarimeter at the $\mathrm{D}$ line of sodium $(\lambda, 589 \mathrm{nM})$. Purity was assessed using ultraperformance liquid chromatography mass spectrometry (UPLC-MS; Acquity PDA detector, Acquity SQ detector, and Acquity UPLC BEH-C18 column, $1.7 \mu \mathrm{m}, 2.1 \times 50 \mathrm{~mm}^{2}$; Waters Corp.). Data were acquired using Masslynx, version 4.1. The flow rate was $0.5 \mathrm{~mL} / \mathrm{min}$, and the gradient started with $95 \% \mathrm{~A}(0.1 \%$ formic acid in $\left.\mathrm{H}_{2} \mathrm{O}\right)$, changed to $95 \% \mathrm{~B}(0.1 \%$ formic acid in acetonitrile), and then returned to $95 \%$ A. The mass spectrometer was operated in the positive-ion mode with electrospray ionization. Verification of enantiopurity was conducted using supercritical fluid chromatography with a Chiralcel OD-H $\left(4.6 \times 250 \mathrm{~mm}^{2}\right)$ or Chiralcel AD-H $(4.6 \times$ $250 \mathrm{~mm}^{2}$ ) column. The compounds were purified to $\geq 95 \%$ purity and assessed using LC/MS by UV/evaporative light scattering detector detection, unless stated otherwise, and efficacy data were obtained only on compounds that were $\geq 95 \%$ pure.

2-Chloro-N-cyclohexylpyrimidin-4-amine (3a). To a suspension of 2,4-dichloropyrimidine 2 (10 mmol, 1.0 equiv) in DCM $(20 \mathrm{~mL}, 0.50 \mathrm{M})$ under a nitrogen atmosphere was added cyclohexylamine ( $20 \mathrm{mmol}, 2.0$ equiv) at rt. After stirring for $16 \mathrm{~h}$ at $\mathrm{rt}$, the reaction mixture was concentrated and purified directly using automated flash chromatography (ethyl acetate (EtOAc)/ hexanes), followed by evaporation, giving $3 a$ as a white solid (1.2 g, 57\%). TLC $R_{f} 0.30$ (30\% EtOAc/hexanes). LC-MS (ESI) $\mathrm{m} /$ $z: 214[\mathrm{M}+\mathrm{H}]^{+} .{ }^{1} \mathrm{H}$ NMR $\left(500 \mathrm{MHz}, \mathrm{CDCl}_{3}\right) \delta 8.02(\mathrm{~s}, 1 \mathrm{H})$, $6.22(\mathrm{~d}, J=6.0 \mathrm{~Hz}, 1 \mathrm{H}), 5.20$ (br s, $1 \mathrm{H}), 3.42$ (br s, $1 \mathrm{H}), 2.08-$ $1.93(\mathrm{~m}, 2 \mathrm{H}), 1.77(\mathrm{dt}, J=13.6,4.0 \mathrm{~Hz}, 2 \mathrm{H}), 1.67(\mathrm{dt}, J=12.9$, $4.1 \mathrm{~Hz}, 1 \mathrm{H}), 1.38-1.44(\mathrm{~m}, 2 \mathrm{H}), 1.32-1.13(\mathrm{~m}, 3 \mathrm{H}) .{ }^{13} \mathrm{C} \mathrm{NMR}$ $\left(126 \mathrm{MHz} \mathrm{CDCl}_{3}\right) \delta 162.76,160.83,157.41,100.54,50.12$, $32.68,25.37,24.51$.

$N^{4}$-Cyclohexyl- $N^{2}$-(3-(pyridin-3-yl)phenyl)pyrimidine-2,4diamine (1). To a mixture of $3 \mathrm{a}(0.10 \mathrm{mmol}, 1.0$ equiv $)$ in $\mathrm{EtOH}$ $(0.50 \mathrm{~mL}, 0.20 \mathrm{M})$ under a nitrogen atmosphere at rt were added 3-(pyridin-3-yl)aniline ( $0.11 \mathrm{mmol}, 1.1$ equiv) and a drop of $1 \mathrm{~N}$ $\mathrm{HCl}$. The reaction mixture was heated to reflux and stirred at that temperature for $3 \mathrm{~h}$; it was then cooled to rt. Purification using automated flash chromatography $(\mathrm{MeOH} / \mathrm{DCM})$ was followed by evaporation, giving 1 as a white film $(0.002 \mathrm{~g}, 6 \%)$. TLC $R_{f} 0.5$ (10\% MeOH/DCM). LC-MS (ESI) $m / z: 346[\mathrm{M}+\mathrm{H}]^{+} .{ }^{1} \mathrm{H}$ NMR (500 MHz, MeOD) $\delta 8.90-8.75(\mathrm{~s}, 1 \mathrm{H}), 8.54(\mathrm{~d}, J=5.0$ $\mathrm{Hz}, 1 \mathrm{H}), 8.11(\mathrm{dt}, J=7.8,2.0 \mathrm{~Hz}, 2 \mathrm{H}), 7.73(\mathrm{~s}, 1 \mathrm{H}), 7.64(\mathrm{~d}, J=$ $10 \mathrm{~Hz}, 1 \mathrm{H}), 7.54(\mathrm{dd}, J=7.9,4.9 \mathrm{~Hz}, 1 \mathrm{H}), 7.41(\mathrm{t}, J=7.9 \mathrm{~Hz}$, $1 \mathrm{H}), 7.25(\mathrm{~d}, J=10 \mathrm{~Hz}, 1 \mathrm{H}), 5.95(\mathrm{~d}, J=6.0 \mathrm{~Hz}, 1 \mathrm{H}), 3.84$ (br s, 
Table 9. Physicochemical Properties of $1,5 e$, and $6 k$

\begin{tabular}{|c|c|c|c|c|}
\hline in vitro properties & & 1 & $5 e$ & $6 \mathrm{k}$ \\
\hline solubility $(\mu \mathrm{M})^{a}$ & $\mathrm{pH}=7.4$ & $0.4 \pm 0.9$ & $26.4 \pm 17.0$ & $56.9 \pm 1.9$ \\
\hline permeability $\left(10^{-6} \mathrm{~cm} / \mathrm{s}\right)^{a}$ & & $1880 \pm 170$ & $2000 \pm 190$ & $1870 \pm 60$ \\
\hline \multirow[t]{4}{*}{ liver microsome stability at $4 \mu \mathrm{M} t_{1 / 2}(\mathrm{~h})^{a}$} & mouse & $0.26 \pm 0.03$ & $0.30 \pm 0.03$ & $0.118 \pm 0.003$ \\
\hline & rat & $1.04 \pm 0.03$ & $1.1 \pm 0.1$ & $0.38 \pm 0.01$ \\
\hline & $\operatorname{dog}$ & $0.7 \pm 0.1$ & $1.2 \pm 0.1$ & $0.90 \pm 0.02$ \\
\hline & human & $0.8 \pm 0.1$ & $2.1 \pm 0.1$ & $1.46 \pm 0.10$ \\
\hline \multirow[t]{4}{*}{ liver microsome stability Clint at $4 \mu \mathrm{M}(\mathrm{mL} / \mathrm{min} / \mathrm{kg})^{a}$} & mouse & $26.5 \pm 1.6$ & $179.6 \pm 19.4$ & 485.3 \\
\hline & rat & $44.9 \pm 1.4$ & $41.7 \pm 2.7$ & 121.98 \\
\hline & $\operatorname{dog}$ & $49.4 \pm 3.9$ & $28.2 \pm 1.9$ & 37.03 \\
\hline & human & $26.6 \pm 2.2$ & $10.0 \pm 0.6$ & 14.22 \\
\hline \multirow[t]{3}{*}{ plasma stability $(\mathrm{h})^{a}$} & mouse & N.D. ${ }^{\bar{b}}$ & $>48$ & $>50$ \\
\hline & rat & N.D. ${ }^{b}$ & $>48$ & $>50$ \\
\hline & human & N.D. ${ }^{b}$ & $>48$ & $>50$ \\
\hline \multirow[t]{3}{*}{ PBS stability $(\mathrm{h})^{a}$} & $\mathrm{pH}=7.4$ & N.D. ${ }^{b}$ & $>48$ & N.D. ${ }^{b}$ \\
\hline & $\mathrm{pH}=5.0$ & N.D. ${ }^{b}$ & $>48$ & N.D. ${ }^{b}$ \\
\hline & $\mathrm{pH}=3.0$ & N.D. ${ }^{b}$ & $>48$ & N.D. ${ }^{b}$ \\
\hline SGF stability $(\mathrm{h})^{a}$ & & N.D. ${ }^{b}$ & $>48$ & N.D. ${ }^{b}$ \\
\hline
\end{tabular}

Table 10. Pharmacokinetic Parameters in Mice for Compound 5e (i.p. Dosing)

\begin{tabular}{llll} 
dose $(\mathrm{mg} / \mathrm{kg})$ & \multicolumn{1}{c}{3} & \multicolumn{1}{c}{5} & 10 \\
$C_{\max }(\mathrm{nM})$ & 376 & 927 & 1650 \\
$T_{\max }(\mathrm{h})$ & 0.083 & 0.083 & 0.25 \\
$T_{1 / 2}(\mathrm{~h})$ & 0.23 & 0.54 & 1 \\
$\mathrm{AUC}(\mathrm{nM} \mathrm{h})$ & 183.3 & 683.1 & 953.3 \\
$V_{\mathrm{z}}(\mathrm{L} / \mathrm{kg})$ & 16.7 & 18.6 & 47.3 \\
$\mathrm{Cl}(\mathrm{L} / \mathrm{h} / \mathrm{kg})$ & 50.7 & 23.8 & 33.1 \\
\hline
\end{tabular}

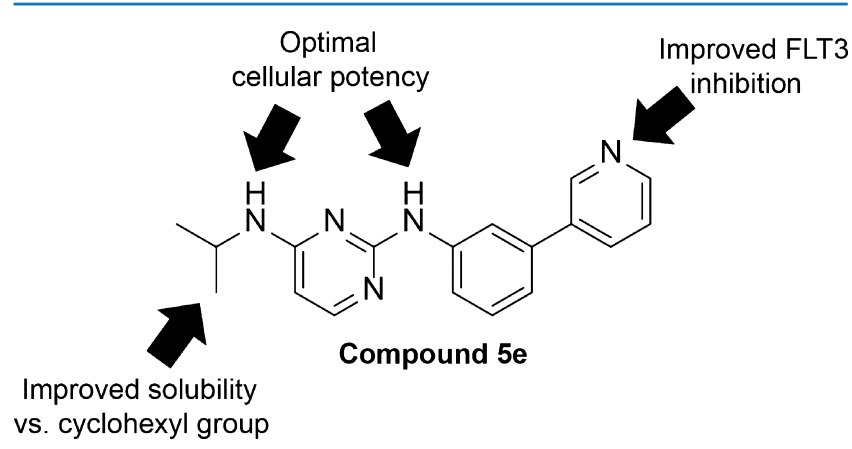

Figure 4. Summary of SAR.

$1 \mathrm{H}), 1.98(\mathrm{dd}, J=9.4,4.8 \mathrm{~Hz}, 2 \mathrm{H}), 1.67-1.69(\mathrm{~m}, 2 \mathrm{H}), 1.56(\mathrm{~s}$, $1 \mathrm{H}), 1.28-1.06(\mathrm{~m}, 5 \mathrm{H}) .{ }^{13} \mathrm{C}$ NMR (126 MHz, MeOD) $\delta$ $162.32,159.48,153.10,147.23,147.10,141.42,137.93,137.65$, $135.32,128.98,124.06,120.04,119.00,117.79,97.65,49.23$, $32.43,25.34,24.72$.

6-Bromo-N-cyclohexylpyridin-2-amine (8). A mixture of 2,6dibromopyridine 7 ( $1.0 \mathrm{mmol}, 1.0$ equiv), cyclohexylamine (1.6 mmol, 1.6 equiv), and triethylamine ( $1.2 \mathrm{mmol}, 1.2$ equiv) was stirred at $100{ }^{\circ} \mathrm{C}$ in $\mathrm{NMP}(1.0 \mathrm{~mL}, 1.0 \mathrm{M})$ under a nitrogen atmosphere for $21 \mathrm{~h}$; it was then cooled to rt. The reaction mixture was partitioned between EtOAc $(3 \mathrm{~mL})$ and water $(3$ $\mathrm{mL})$. The organic phase was washed with brine $(3 \mathrm{~mL})$, dried over magnesium sulfate, filtered, and concentrated. Purification using automated flash chromatography (EtOAc/hexanes) was followed by evaporation, giving 8 as a colorless oil (0.026 g, $10 \%$ ). TLC $R_{f} 0.50$ ( $10 \%$ EtOAc/hexanes). LC-MS (ESI) $\mathrm{m} / z$ : $257[\mathrm{M}+\mathrm{H}]^{+} .{ }^{1} \mathrm{H}$ NMR $\left(400 \mathrm{MHz}, \mathrm{CDCl}_{3}\right) \delta 7.22-7.07(\mathrm{~m}$,
$1 \mathrm{H}), 6.60(\mathrm{~d}, J=7.4 \mathrm{~Hz}, 1 \mathrm{H}), 6.18(\mathrm{~d}, J=8.2 \mathrm{~Hz}, 1 \mathrm{H}), 4.64-4.47$ (br s, $1 \mathrm{H}), 3.41-3.26(\mathrm{~m}, 1 \mathrm{H}), 1.89-1.96(\mathrm{~m}, 2 \mathrm{H}), 1.72-1.61$ $(\mathrm{m}, 2 \mathrm{H}), 1.53-1.59(\mathrm{~m}, 1 \mathrm{H}), 1.39-1.23(\mathrm{~m}, 2 \mathrm{H}), 1.23-1.05(\mathrm{~m}$, $3 \mathrm{H}) .{ }^{13} \mathrm{C}$ NMR $\left(101 \mathrm{MHz}, \mathrm{CDCl}_{3}\right) \delta 158.14,140.32,139.48$, 115.22, 104.14, 50.36, 32.99, 25.66, 24.69.

$N^{2}$-Cyclohexyl- $N^{6}-(3-($ pyridin-3-yl)phenyl)pyridine-2,6-diamine (9). A mixture of 8 (0.078 mmol, 1.0 equiv), 3-(pyridin-3yl)aniline ( $0.10 \mathrm{mmol}, 1.3$ equiv), sodium $t$-butoxide $(0.39$ mmol, 5.0 equiv), $\mathrm{Pd}_{2}(\mathrm{dba})_{3}(0.005 \mathrm{mmol}, 7 \mathrm{~mol} \%)$, and $R$ BINAP $(0.012 \mathrm{mmol}, 15 \mathrm{~mol} \%)$ was stirred in toluene $(1.0 \mathrm{~mL}$, $0.078 \mathrm{M}$ ) at $80^{\circ} \mathrm{C}$ under a nitrogen atmosphere for $16 \mathrm{~h}$; it was then cooled to rt. The reaction mixture was then diluted with EtOAc $(3 \mathrm{~mL})$ and washed with brine $(2 \times 3 \mathrm{~mL})$. The organic phase was dried over magnesium sulfate, filtered, and concentrated. Purification using automated flash chromatography (EtOAc/hexanes) was followed by evaporation, giving 9 as a yellow oil $(0.015 \mathrm{~g}, 56 \%)$. TLC $R_{f} 0.70$ (80\% EtOAc/hexanes). LC-MS (ESI) $m / z: 345[\mathrm{M}+\mathrm{H}]^{+}$. ${ }^{1} \mathrm{H}$ NMR $(500 \mathrm{MHz}$, $\mathrm{MeOD}) \delta 8.79(\mathrm{~s}, 1 \mathrm{H}), 8.51(\mathrm{~s}, 1 \mathrm{H}), 8.13-7.96(\mathrm{~m}, 2 \mathrm{H}), 7.60(\mathrm{~d}$, $J=8.4 \mathrm{~Hz}, 1 \mathrm{H}), 7.58-7.48(\mathrm{~m}, 1 \mathrm{H}), 7.34(\mathrm{t}, J=8.0 \mathrm{~Hz}, 1 \mathrm{H})$, $7.26-7.18(\mathrm{~m}, 1 \mathrm{H}), 7.11(\mathrm{~d}, J=7.7 \mathrm{~Hz}, 1 \mathrm{H}), 6.02(\mathrm{~d}, J=8.1 \mathrm{~Hz}$, $1 \mathrm{H}), 5.90(\mathrm{~d}, J=8.0 \mathrm{~Hz}, 1 \mathrm{H}), 3.77-3.59(\mathrm{~m}, 1 \mathrm{H}), 2.11-1.93(\mathrm{~m}$, $2 \mathrm{H}), 1.75-1.59(\mathrm{~m}, 2 \mathrm{H}), 1.54-1.68(\mathrm{~m}, 1 \mathrm{H}), 1.15-1.22(\mathrm{~m}$, $5 \mathrm{H}) .{ }^{13} \mathrm{C}$ NMR (126 MHz, MeOD) $\delta 157.57,157.53,154.95$, 154.91, 147.07, 143.20, 138.20, 138.10, 138.00, 137.89, 137.66, $135.29,129.00,124.03,118.50,117.77,116.36,97.90,97.88$, $96.85,96.83,49.80,33.06,25.60,24.87$.

2-Chloro-N-cyclohexylpyridin-4-amine (11). A mixture of 4bromo-2-chloropyridine 10 (4.0 mmol, 1.0 equiv), cyclohexylamine (6.4 mmol, 1.6 equiv), and triethylamine ( $4.8 \mathrm{mmol}, 1.2$ equiv) was stirred at $80{ }^{\circ} \mathrm{C}$ in NMP $(2.0 \mathrm{~mL}, 2.0 \mathrm{M})$ under a nitrogen atmosphere for $16 \mathrm{~h}$. The reaction mixture was cooled to $\mathrm{rt}$ and then partitioned between EtOAc $(3 \mathrm{~mL})$ and water $(3$ $\mathrm{mL})$. The organic phase was washed with brine $(3 \mathrm{~mL})$, dried over magnesium sulfate, filtered, and concentrated. Purification using automated flash chromatography (EtOAc/hexanes) was followed by evaporation, giving 11 as an orange solid $(0.123 \mathrm{~g}$, $15 \%)$. TLC $R_{f} 0.40$ (30\% EtOAc/hexanes). LC-MS (ESI) $m / z$ : $211[\mathrm{M}+\mathrm{H}]^{+} .{ }^{1} \mathrm{H}$ NMR $\left(400 \mathrm{MHz}, \mathrm{CDCl}_{3}\right) \delta 7.92(\mathrm{~d}, J=5.8$ $\mathrm{Hz}, 1 \mathrm{H}), 6.43(\mathrm{~s}, 1 \mathrm{H}), 6.33$ (dd, $J=5.8,2.2 \mathrm{~Hz}, 1 \mathrm{H}), 4.26$ (d, $J=$ $7.8 \mathrm{~Hz}, 1 \mathrm{H}), 3.34-3.22(\mathrm{~m}, 1 \mathrm{H}), 2.13-1.94(\mathrm{~m}, 2 \mathrm{H}), 1.88-1.74$ 
$(\mathrm{m}, 2 \mathrm{H}), 1.74-1.61(\mathrm{~m}, 1 \mathrm{H}), 1.47-1.32(\mathrm{~m}, 2 \mathrm{H}), 1.32-1.12(\mathrm{~m}$, $3 \mathrm{H}) .{ }^{13} \mathrm{C} \mathrm{NMR}\left(101 \mathrm{MHz}, \mathrm{CDCl}_{3}\right) \delta 154.37,152.16,149.16$, 107.41, 106.04, 51.03, 32.81, 25.54, 24.72.

$N^{4}$-cyclohexyl- $N^{2}$-(3-(pyridin-3-yl)phenyl)pyridine-2,4-diamine (13). A mixture of 11 ( $0.20 \mathrm{mmol}, 1.0$ equiv), 3-(pyridin-3yl)aniline ( $0.25 \mathrm{mmol}, 1.3$ equiv), sodium $t$-butoxide $(0.97$ mmol, 5.0 equiv), $\mathrm{Pd}_{2}(\mathrm{dba})_{3}(0.014 \mathrm{mmol}, 7 \mathrm{~mol} \%)$, and $R$ BINAP $(0.029 \mathrm{mmol}, 0.15 \mathrm{mmol})$ was stirred in DMF $(1.0 \mathrm{~mL}$, $0.20 \mathrm{M})$ at $140{ }^{\circ} \mathrm{C}$ under microwave irradiation at $35 \mathrm{~W}$ for $1 \mathrm{~h}$. The reaction mixture was then cooled to rt, diluted with EtOAc $(5 \mathrm{~mL})$, and washed with brine $(5 \mathrm{~mL})$. The organic phase was dried over magnesium sulfate, filtered, and concentrated. Purification using automated flash chromatography $(\mathrm{MeOH} /$ $\mathrm{CH}_{2} \mathrm{Cl}_{2}$ ) was followed by evaporation, giving 13 as a brown oil (0.016 g, 24\%). TLC $R_{f} 0.3\left(10 \% \mathrm{MeOH} / \mathrm{CH}_{2} \mathrm{Cl}_{2}\right)$. LC-MS (ESI) $\mathrm{m} / z: 345[\mathrm{M}+\mathrm{H}]^{+} .{ }^{1} \mathrm{H}$ NMR $\left(400 \mathrm{MHz}, \mathrm{CDCl}_{3}\right) \delta 8.81$ $(\mathrm{s}, 1 \mathrm{H}), 8.61-8.56(\mathrm{~m}, 1 \mathrm{H}), 7.86(\mathrm{dt}, J=7.9,2.3 \mathrm{~Hz}, 1 \mathrm{H}), 7.57-$ $7.50(\mathrm{~m}, 1 \mathrm{H}), 7.48-7.42(\mathrm{~m}, 2 \mathrm{H}), 7.35-7.38(\mathrm{~m}, 1 \mathrm{H}), 7.27-$ $7.32(\mathrm{~m}, 2 \mathrm{H}), 6.12(\mathrm{dd}, J=6.6,2.1 \mathrm{~Hz}, 1 \mathrm{H}), 6.02(\mathrm{~s}, 1 \mathrm{H}), 5.21(\mathrm{~d}$, $J=7.6 \mathrm{~Hz}, 1 \mathrm{H}), 3.27-3.14(\mathrm{~m}, 1 \mathrm{H}), 1.92-1.97$ (m, 2H), 1.76$1.68(\mathrm{~m}, 2 \mathrm{H}), 1.59-1.61(\mathrm{~m}, 1 \mathrm{H}), 1.16-1.30(\mathrm{~m}, 5 \mathrm{H}) .{ }^{13} \mathrm{C} \mathrm{NMR}$ $\left(101 \mathrm{MHz}, \mathrm{CDCl}_{3}\right) \delta 155.68,153.72,148.79,148.15,140.43$, $139.34,139.26,135.98,134.40,130.21,123.64,123.14,121.73$, 120.74, 102.29, 87.65, 51.48, 32.59, 25.36, 24.58.

4-Bromo-N-cyclohexylpyridin-2-amine (12). A mixture of 4bromo-2-chloropyridine 10 ( $1.0 \mathrm{mmol}, 1.0$ equiv), cyclohexylamine ( $1.6 \mathrm{mmol}, 1.6$ equiv), and triethylamine ( $1.2 \mathrm{mmol}, 1.2$ equiv) was stirred at $80^{\circ} \mathrm{C}$ in NMP $(1.0 \mathrm{~mL}, 1.0 \mathrm{M})$ under a nitrogen atmosphere for $16 \mathrm{~h}$. The reaction mixture was cooled to $\mathrm{rt}$ and then partitioned between EtOAc $(3 \mathrm{~mL})$ and water $(3$ $\mathrm{mL})$. The organic phase was washed with brine $(3 \mathrm{~mL})$, dried over magnesium sulfate, filtered, and concentrated. Purification using automated flash chromatography (EtOAc/hexanes) was followed by evaporation, giving $\mathbf{1 2}$ as a colorless oil (0.006 g, $2 \%)$. TLC $R_{f} 0.75$ (30\% EtOAc/hexanes). LC-MS (ESI) $m / z$ : $255\left[\mathrm{M}+\mathrm{H}^{+} .{ }^{1} \mathrm{H}\right.$ NMR $\left(400 \mathrm{MHz}, \mathrm{CDCl}_{3}\right) \delta 7.88(\mathrm{~d}, J=5.5$ $\mathrm{Hz}, 1 \mathrm{H}), 6.69(\mathrm{dd}, J=5.5,1.6 \mathrm{~Hz}, 1 \mathrm{H}), 6.55(\mathrm{~d}, J=1.6 \mathrm{~Hz}, 1 \mathrm{H})$, $4.64(\mathrm{~s}, 1 \mathrm{H}), 3.59-3.42(\mathrm{~m}, 1 \mathrm{H}), 2.08-1.98(\mathrm{~m}, 2 \mathrm{H}), 1.85-1.71$ $(\mathrm{m}, 2 \mathrm{H}), 1.67(\mathrm{dt}, J=12.7,3.9 \mathrm{~Hz}, 1 \mathrm{H}), 1.53-1.33(\mathrm{~m}, 2 \mathrm{H})$, $1.33-1.14(\mathrm{~m}, 3 \mathrm{H}) .{ }^{13} \mathrm{C} \mathrm{NMR}\left(101 \mathrm{MHz}, \mathrm{CDCl}_{3}\right) \delta 158.62$, 148.77, 133.60, 115.61, 109.24, 50.28, 33.17, 25.68, 24.79.

$N^{2}$-Cyclohexyl- $N^{4}$-(3-(pyridin-3-yl)phenyl)pyridine-2,4-diamine (14). A mixture of 12 ( $0.067 \mathrm{mmol}, 1.0$ equiv), 3-(pyridin3-yl)aniline ( $0.087 \mathrm{mmol}, 1.3$ equiv), sodium $t$-butoxide $(0.33$ mmol, 5.0 equiv), $\mathrm{Pd}_{2}(\mathrm{dba})_{3}(0.0047 \mathrm{mmol}, 7 \mathrm{~mol} \%)$, and $R$ BINAP $(0.010 \mathrm{mmol}, 15 \mathrm{~mol} \%)$ was stirred in toluene $(1.0 \mathrm{~mL}$, $0.067 \mathrm{M})$ at $80{ }^{\circ} \mathrm{C}$ under a nitrogen atmosphere. After $16 \mathrm{~h}$, the reaction mixture was cooled to rt, diluted with EtOAc $(3 \mathrm{~mL})$, and washed with brine $(2 \times 3 \mathrm{~mL})$. The organic phase was dried over magnesium sulfate, filtered, and concentrated. Purification using automated flash chromatography ( $\mathrm{MeOH} / \mathrm{DCM})$ was followed by evaporation, giving 14 as a white oil (0.002 g, 9\%). TLC $R_{f} 0.2$ (10\% MeOH/DCM). LC-MS (ESI) $m / z: 345[\mathrm{M}+$ $\mathrm{H}]^{+} .{ }^{1} \mathrm{H}$ NMR (500 MHz, MeOD) $\delta 8.84(\mathrm{~s}, 1 \mathrm{H}), 8.58(\mathrm{~s}, 1 \mathrm{H})$, $8.14(\mathrm{~d}, J=5.0 \mathrm{~Hz}, 1 \mathrm{H}), 7.68-7.46(\mathrm{~m}, 5 \mathrm{H}), 7.39-7.30(\mathrm{~d}, J=$ $5.0 \mathrm{~Hz}, 1 \mathrm{H}), 6.44(\mathrm{dd}, J=7.0,2.2 \mathrm{~Hz}, 1 \mathrm{H}), 6.25(\mathrm{~s}, 1 \mathrm{H}), 3.40-$ $3.44(\mathrm{~m}, 1 \mathrm{H}), 1.99(\mathrm{~d}, J=15.0 \mathrm{~Hz}, 2 \mathrm{H}), 1.77-1.83(\mathrm{~m}, 2 \mathrm{H}), 1.68$ $(\mathrm{dt}, J=12.8,3.8 \mathrm{~Hz}, 1 \mathrm{H}), 1.37-1.46(\mathrm{~m}, 2 \mathrm{H}), 1.24-1.34(\mathrm{~m}$, $3 \mathrm{H}) .{ }^{13} \mathrm{C}$ NMR (126 MHz, MeOD) $\delta 154.89,153.53,147.83$, $146.98,139.59$, 138.87, 136.95, 136.53, 135.22, 130.27, 124.16, 123.63, 122.39, 121.05, 102.81, 88.10, 50.49, 32.29, 25.05, 24.37.

2-Chloro-N-cyclohexyl-N-methylpyrimidin-4-amine (15). A mixture of 2,4-dichloropyrimidine 2 (5.0 mmol, 1.0 equiv), $\mathrm{N}$ - methylaminocyclohexane $(5.0 \mathrm{mmol}, 1.0$ equiv), and triethylamine ( $5.5 \mathrm{mmol}$, 1.1 equiv) was stirred in $\mathrm{EtOH}$ (7.0 mL, 0.71 $\mathrm{M})$ under a nitrogen atmosphere at reflux temperature for $5 \mathrm{~h}$. The reaction mixture was then cooled to $\mathrm{rt}$ and concentrated. Purification using automated flash chromatography (EtOAc/ hexanes) was followed by evaporation, giving $\mathbf{1 5}$ as a white solid (0.67 g, 59\%). TLC $R_{f} 0.50$ (30\% EtOAc/hexanes). LC-MS (ESI) $m / z: 228[\mathrm{M}+\mathrm{H}]^{+} .{ }^{1} \mathrm{H}$ NMR $\left(500 \mathrm{MHz}, \mathrm{CDCl}_{3}\right) \delta 8.00$ $(\mathrm{d}, J=6.2 \mathrm{~Hz}, 1 \mathrm{H}), 6.46-6.17(\mathrm{~m}, 1 \mathrm{H}), 4.72(\mathrm{br} \mathrm{s}, 1 \mathrm{H}), 2.93(\mathrm{br}$ $\mathrm{s}, 3 \mathrm{H}), 1.97-1.80(\mathrm{~m}, 2 \mathrm{H}), 1.83-1.61(\mathrm{~m}, 3 \mathrm{H}), 1.43-1.52(\mathrm{~m}$, $4 \mathrm{H}), 1.23-1.05(\mathrm{~m}, 1 \mathrm{H}) .{ }^{13} \mathrm{C}$ NMR $\left(126 \mathrm{MHz}, \mathrm{CDCl}_{3}\right) \delta$ $162.58,160.52,156.66,101.31,29.82,25.63,25.48$.

$N^{4}$-Cyclohexyl- $N^{4}$-methyl- $N^{2}-(3-($ pyridin-3-yl)phenyl)pyrimidine-2,4-diamine (16). To a mixture of $15(0.10 \mathrm{mmol}$, 1.0 equiv) in $\mathrm{EtOH}(0.50 \mathrm{~mL}, 0.20 \mathrm{M})$ under a nitrogen atmosphere at $\mathrm{rt}$ were added 3-(3-pyridyl)aniline $(0.11 \mathrm{mmol}$, 1.1 equiv) and a drop of $1 \mathrm{~N} \mathrm{HCl}$. The reaction mixture was heated to reflux and stirred at that temperature for $3 \mathrm{~h}$; it was then cooled to rt. Purification using automated flash chromatography (EtOAc/hexanes) was followed by evaporation, giving $\mathbf{1 6}$ as a colorless oil $(0.009 \mathrm{~g}, 25 \%)$. TLC $R_{f} 0.1$ (70\% EtOAc/hexanes). LC-MS (ESI) $m / z: 360[\mathrm{M}+\mathrm{H}]^{+}$. ${ }^{1} \mathrm{H}$ NMR $(500 \mathrm{MHz}$, $\left.\mathrm{CDCl}_{3}\right) \delta 8.86(\mathrm{~d}, J=2.3 \mathrm{~Hz}, 1 \mathrm{H}), 8.58(\mathrm{dd}, J=4.8,1.6 \mathrm{~Hz}, 1 \mathrm{H})$, $7.94-7.84(\mathrm{~m}, 2 \mathrm{H}), 7.61(\mathrm{~d}, J=8.7 \mathrm{~Hz}, 2 \mathrm{H}), 7.40(\mathrm{t}, J=7.9 \mathrm{~Hz}$, $1 \mathrm{H}), 7.35(\mathrm{dd}, J=7.9,4.8 \mathrm{~Hz}, 1 \mathrm{H}), 7.19(\mathrm{~d}, J=5.0,1 \mathrm{H}), 5.98(\mathrm{~d}, J$ $=6.3 \mathrm{~Hz}, 1 \mathrm{H}), 4.28(\mathrm{br} \mathrm{s}, 2 \mathrm{H}), 2.92(\mathrm{~s}, 3 \mathrm{H}), 1.84-1.72(\mathrm{~m}, 2 \mathrm{H})$, $1.70(\mathrm{~d}, J=3.9 \mathrm{~Hz}, 2 \mathrm{H}), 1.67-1.56(\mathrm{~m}, 1 \mathrm{H}), 1.45(\mathrm{q}, J=11.9$, $11.1 \mathrm{~Hz}, 2 \mathrm{H}), 1.19-1.25(\mathrm{~m}, 2 \mathrm{H}), 1.14-1.00(\mathrm{~m}, 1 \mathrm{H}) .{ }^{13} \mathrm{C} \mathrm{NMR}$ $\left(126 \mathrm{MHz}, \mathrm{CDCl}_{3}\right) \delta 162.03,158.45,154.29,148.46,148.44$, $140.76,138.53,137.01,134.49,129.36,123.47,120.79,118.85$, $117.89,95.37,54.65,30.01,29.47,25.75,25.55$.

$N^{4}$-Cyclohexyl- $N^{2}$-methyl-N²-(3-(pyridin-3-yl)phenyl)pyrimidine-2,4-diamine (21). To a mixture of $3 \mathrm{a}(0.10 \mathrm{mmol}$, 1.0 equiv) in $\mathrm{EtOH}(0.50 \mathrm{~mL}, 0.20 \mathrm{M})$ under a nitrogen atmosphere at rt were added $\mathrm{N}$-methyl-3-(pyridin-3-yl)aniline $(0.11 \mathrm{mmol}, 1.1$ equiv) and a drop of $1 \mathrm{~N} \mathrm{HCl}$. The reaction mixture was heated to reflux and stirred at that temperature for 2 $h$; it was then cooled to rt. Purification using automated flash chromatography (MeOH/DCM) was followed by evaporation, giving 21 as a red oil $(0.002 \mathrm{~g}, 6 \%)$. TLC $R_{f} 0.2(10 \% \mathrm{MeOH} /$ DCM). LC-MS (ESI) $m / z: 360[\mathrm{M}+\mathrm{H}]^{+} .{ }^{1} \mathrm{H}$ NMR $(400 \mathrm{MHz}$, MeOD) $\delta 10.18(\mathrm{t}, J=1.7 \mathrm{~Hz}, 1 \mathrm{H}), 10.00(\mathrm{dt}, J=6.3,1.4 \mathrm{~Hz}$, $1 \mathrm{H}), 9.05(\mathrm{dt}, J=8.1,1.6 \mathrm{~Hz}, 1 \mathrm{H}), 8.31(\mathrm{dd}, J=8.1,6.3 \mathrm{~Hz}, 1 \mathrm{H})$, $8.25(\mathrm{~d}, J=6.0 \mathrm{~Hz}, 1 \mathrm{H}), 7.38(\mathrm{t}, J=7.9 \mathrm{~Hz}, 1 \mathrm{H}), 7.03-7.08(\mathrm{~m}$, $1 \mathrm{H}), 7.02(\mathrm{t}, J=2.1 \mathrm{~Hz}, 1 \mathrm{H}), 6.89-6.79(\mathrm{~m}, 1 \mathrm{H}), 6.73(\mathrm{~d}, J=6.0$ $\mathrm{Hz}, 1 \mathrm{H}), 4.22-3.97(\mathrm{~m}, 1 \mathrm{H}), 2.89(\mathrm{~s}, 3 \mathrm{H}), 2.11(\mathrm{~d}, J=13.3 \mathrm{~Hz}$, $2 \mathrm{H}), 1.87(\mathrm{dt}, J=13.0,3.7 \mathrm{~Hz}, 2 \mathrm{H}), 1.74(\mathrm{dt}, J=12.9,3.8 \mathrm{~Hz}$, $1 \mathrm{H}), 1.58-1.27(\mathrm{~m}, 5 \mathrm{H}) .{ }^{13} \mathrm{C}$ NMR (101 MHz, MeOD) $\delta$ $163.12,149.09$, 148.41, 139.93, 137.95, 136.60, 131.55, 127.82, 127.13, 126.40, 125.55, 76.82, 51.05, 39.06, 33.48, 26.68, 26.09.

$N^{4}$-Cyclohexyl- $N^{2}, N^{4}$-dimethyl- $N^{2}$-(3-(pyridin-3-yl)phenyl)pyrimidine-2,4-diamine (17). To a mixture of $15(0.10 \mathrm{mmol}$, 1.0 equiv $)$ in $\mathrm{EtOH}(0.50 \mathrm{~mL}, 0.20 \mathrm{M})$ under a nitrogen atmosphere at rt were added $\mathrm{N}$-methyl-3-(pyridin-3-yl)aniline ( $0.11 \mathrm{mmol}, 1.1$ equiv) and a drop of $1 \mathrm{~N} \mathrm{HCl}$. The reaction mixture was heated to reflux and stirred at that temperature for 3 $\mathrm{h}$; it was then cooled to rt. Purification using automated flash chromatography ( $\mathrm{MeOH} / \mathrm{DCM})$ was followed by evaporation, giving 17 as an orange oil $(0.010 \mathrm{~g}, 27 \%)$. TLC $R_{f} 0.4(10 \%$ $\mathrm{MeOH} / \mathrm{DCM})$. LC-MS (ESI) $m / z: 374[\mathrm{M}+\mathrm{H}]^{+} .{ }^{1} \mathrm{H}$ NMR $(500 \mathrm{MHz}, \mathrm{MeOD}) \delta 10.19(\mathrm{~s}, 1 \mathrm{H}), 10.01(\mathrm{~d}, J=7.4 \mathrm{~Hz}, 1 \mathrm{H})$, $9.06(\mathrm{~d}, J=8.1 \mathrm{~Hz}, 1 \mathrm{H}), 8.42(\mathrm{t}, J=7.2 \mathrm{~Hz}, 1 \mathrm{H}), 8.33(\mathrm{t}, J=7.2$ $\mathrm{Hz}, 1 \mathrm{H}), 7.38(\mathrm{t}, J=7.9 \mathrm{~Hz}, 1 \mathrm{H}), 7.08(\mathrm{~d}, J=7.5 \mathrm{~Hz}, 1 \mathrm{H}), 7.03(\mathrm{~s}$, 
$J=2.0 \mathrm{~Hz}, 1 \mathrm{H}), 6.98-6.88(\mathrm{~m}, 1 \mathrm{H}), 6.84(\mathrm{~d}, J=5.0 \mathrm{~Hz}, 1 \mathrm{H})$, $3.95(\mathrm{~s}, 1 \mathrm{H}), 3.09-3.26(\mathrm{~m}, 3 \mathrm{H}), 2.89(\mathrm{~s}, 3 \mathrm{H}), 2.02-1.89(\mathrm{~m}$, $2 \mathrm{H}), 1.83-1.86(\mathrm{~m}, 2 \mathrm{H}), 1.67-1.77(\mathrm{~m}, 3 \mathrm{H}), 1.56(\mathrm{qt}, J=13.2$, $3.6 \mathrm{~Hz}, 2 \mathrm{H}), 1.38-1.23(\mathrm{~m}, 1 \mathrm{H}) .{ }^{13} \mathrm{C} \mathrm{NMR}(126 \mathrm{MHz}, \mathrm{MeOD})$ $\delta 162.46,156.01,155.64,151.30,146.51,141.80,138.18,137.88$, $134.24,130.11,127.39,114.72,114.23,109.54,105.17,55.20$, 29.23, 29.05, 25.53, 25.14 .

2-Chloro-4-(cyclohexyloxy)pyrimidine (18). To a solution of cyclohexanol (6.0 mmol, 1.2 equiv $)$ in DMF $(10 \mathrm{~mL}, 0.60 \mathrm{M})$ at 0 ${ }^{\circ} \mathrm{C}$ was added $\mathrm{NaH}$ as a $60 \%$ suspension in mineral oil $(7.5 \mathrm{mmol}$, 1.5 equiv). After stirring for $20 \mathrm{~min}, 2$,4-dichloropyrimidine 2 ( $5.0 \mathrm{mmol}, 1.0$ equiv) was added at $0{ }^{\circ} \mathrm{C}$ to one portion. The reaction mixture was then stirred under a nitrogen atmosphere while warming to rt over $16 \mathrm{~h}$. Brine $(20 \mathrm{~mL})$ was added to the reaction mixture; then, the mixture was extracted into EtOAc (2 $\times 20 \mathrm{~mL}$ ). The organic phase was dried over magnesium sulfate, filtered, and concentrated. Purification using automated flash chromatography (EtOAc/hexanes) was followed by evaporation, giving 18 as a white solid (0.031 g, 3\%). TLC $R_{f} 0.35(10 \%$ EtOAc/hexanes). LC-MS (ESI) $m / z: 213[\mathrm{M}+\mathrm{H}]^{+} .{ }^{1} \mathrm{H}$ NMR $\left(400 \mathrm{MHz}, \mathrm{CDCl}_{3}\right) \delta 8.18(\mathrm{~d}, J=5.7 \mathrm{~Hz}, 1 \mathrm{H}), 6.53(\mathrm{~d}, J=5.7 \mathrm{~Hz}$, $1 \mathrm{H}), 5.17-4.97(\mathrm{~m}, 1 \mathrm{H}), 2.00-1.84(\mathrm{~m}, 2 \mathrm{H}), 1.78-1.63(\mathrm{~m}$, $2 \mathrm{H}), 1.56-1.49(\mathrm{~m}, 2 \mathrm{H}), 1.35-1.48(\mathrm{~m}, 3 \mathrm{H}), 1.19-1.34(\mathrm{~m}$, 1H). ${ }^{13} \mathrm{C} \mathrm{NMR}\left(101 \mathrm{MHz}, \mathrm{CDCl}_{3}\right) \delta 170.02,160.21,158.63$, 107.61, 75.52, 31.32, 25.36, 23.60.

4-(Cyclohexyloxy)-N-(3-(pyridin-3-yl)phenyl)pyrimidin-2amine (19). To a mixture of $\mathbf{1 8}(0.10 \mathrm{mmol}, 1.0$ equiv) in $\mathrm{EtOH}$ $(0.50 \mathrm{~mL}, 0.20 \mathrm{M})$ under a nitrogen atmosphere at rt were added 3-(pyridin-3-yl)aniline ( $0.11 \mathrm{mmol}, 1.1$ equiv) and a drop of $1 \mathrm{~N}$ $\mathrm{HCl}$. The reaction mixture was heated to reflux and stirred at that temperature for $3 \mathrm{~h}$; it was then cooled to rt. Purification using automated flash chromatography $(\mathrm{MeOH} / \mathrm{DCM})$ was followed by evaporation, giving 19 as an orange oil $(0.003 \mathrm{~g}, 9 \%)$. TLC $R_{f}$ $0.3(10 \% \mathrm{MeOH} / \mathrm{DCM})$. LC-MS (ESI) $m / z: 347[\mathrm{M}+\mathrm{H}]^{+} .{ }^{1} \mathrm{H}$ NMR (500 MHz, MeOD) $\delta 10.17(\mathrm{~d}, J=1.8 \mathrm{~Hz}, 1 \mathrm{H}), 10.02(\mathrm{dt}$, $J=6.3,1.8 \mathrm{~Hz}, 1 \mathrm{H}), 9.09(\mathrm{dt}, J=8.1,2.2 \mathrm{~Hz}, 1 \mathrm{H}), 8.79(\mathrm{t}, J=6.1$ $\mathrm{Hz}, 1 \mathrm{H}), 8.37(\mathrm{dd}, J=8.0,6.3 \mathrm{~Hz}, 1 \mathrm{H}), 7.36(\mathrm{t}, J=7.8 \mathrm{~Hz}, 1 \mathrm{H})$, $7.27-7.07(\mathrm{~m}, 3 \mathrm{H}), 6.94(\mathrm{dd}, J=8.0,2.3 \mathrm{~Hz}, 1 \mathrm{H}), 5.40-5.45(\mathrm{~m}$, $1 \mathrm{H}), 2.13-2.18(\mathrm{~m}, 2 \mathrm{H}), 1.96-1.83(\mathrm{~m}, 2 \mathrm{H}), 1.70-1.78(\mathrm{~m}$, $3 \mathrm{H}), 1.65-1.53(\mathrm{~m}, 2 \mathrm{H}), 1.44-1.49(\mathrm{~m}, 1 \mathrm{H}) .{ }^{13} \mathrm{C}$ NMR (126 $\mathrm{MHz}, \mathrm{MeOD}) \delta 171.00,159.02,154.69,149.51,147.06,141.78$, $138.57,138.14,134.01,130.30,127.67,116.71,115.78,112.77$, 111.06, 77.04, 30.86, 25.03, 23.13.

N-Cyclohexyl-2-(3-(pyridin-3-yl)phenoxy)pyrimidin-4amine (22). To a mixture of $3 \mathrm{a}(0.10 \mathrm{mmol}, 1.0$ equiv $)$ in $\mathrm{EtOH}$ $(0.50 \mathrm{~mL}, 0.20 \mathrm{M})$ under a nitrogen atmosphere at rt were added 3-(pyridin-3-yl)phenol ( $0.11 \mathrm{mmol}, 1.1$ equiv) and a drop of $1 \mathrm{~N}$ $\mathrm{HCl}$. The reaction mixture was heated to reflux and stirred at that temperature for $16 \mathrm{~h}$; it was then cooled to rt. Purification using automated flash chromatography $(\mathrm{MeOH} / \mathrm{DCM})$ was followed by evaporation, giving 22 as a yellow oil $(0.028 \mathrm{~g}, 81 \%)$. TLC $R_{f}$ $0.1(10 \% \mathrm{MeOH} / \mathrm{DCM})$. LC-MS (ESI) $m / z: 347[\mathrm{M}+\mathrm{H}]^{+} .{ }^{1} \mathrm{H}$ $\operatorname{NMR}(500 \mathrm{MHz}, \mathrm{MeOD}) \delta 10.19(\mathrm{~d}, J=9.9 \mathrm{~Hz}, 1 \mathrm{H}), 10.01(\mathrm{~d}, J$ $=10.0 \mathrm{~Hz}, 1 \mathrm{H}), 9.05(\mathrm{~d}, J=9.1 \mathrm{~Hz}, 1 \mathrm{H}), 8.29(\mathrm{dt}, J=41.0,6.6 \mathrm{~Hz}$, $2 \mathrm{H}), 7.46(\mathrm{t}, J=9.8 \mathrm{~Hz}, 1 \mathrm{H}), 7.40-7.23(\mathrm{~m}, 2 \mathrm{H}), 7.04(\mathrm{~d}, J=9.0$ $\mathrm{Hz}, 1 \mathrm{H}), 6.82-6.65(\mathrm{~m}, 1 \mathrm{H}), 4.10(\mathrm{~m}, 1 \mathrm{H}), 2.11(\mathrm{~m}, 2 \mathrm{H}), 2.00-$ $1.81(\mathrm{~m}, 2 \mathrm{H}), 1.73(\mathrm{~m}, 1 \mathrm{H}), 1.64-1.26(\mathrm{~m}, 5 \mathrm{H}) .{ }^{13} \mathrm{C} \mathrm{NMR}(126$ $\mathrm{MHz}, \mathrm{MeOD}) \delta 162.96,158.68,154.25,146.51,140.85,138.31$, $137.88,134.78,130.67,127.49,117.99,117.09,113.84,107.95$, 49.88, 31.93, 25.33, 24.58 .

4-(Cyclohexyloxy)-2-(3-(pyridin-3-yl)phenoxy)pyrimidine (20). To a mixture of 18 ( $0.10 \mathrm{mmol}, 1.0$ equiv) in $\mathrm{EtOH}$ (0.50 $\mathrm{mL}, 0.20 \mathrm{M})$ under a nitrogen atmosphere at rt were added 3- (pyridin-3-yl)phenol ( $0.11 \mathrm{mmol}, 1.1$ equiv) and a drop of $1 \mathrm{~N}$ $\mathrm{HCl}$. The reaction mixture was heated to reflux and stirred at that temperature for $16 \mathrm{~h}$; it was then cooled to rt. Purification using automated flash chromatography $(\mathrm{MeOH} / \mathrm{DCM})$ was followed by evaporation, giving 20 as a yellow solid $(0.018 \mathrm{~g}, 52 \%)$. TLC $R_{f} 0.3(10 \% \mathrm{MeOH} / \mathrm{DCM})$. LC-MS (ESI) $m / z: 348[\mathrm{M}+\mathrm{H}]^{+}$. ${ }^{1} \mathrm{H}$ NMR (400 MHz, MeOD) $\delta 10.19(\mathrm{t}, J=1.7 \mathrm{~Hz}, 1 \mathrm{H}), 10.05$ (dt, $J=6.4,1.4 \mathrm{~Hz}, 1 \mathrm{H}), 9.13(\mathrm{ddd}, J=8.1,2.0,1.2 \mathrm{~Hz}, 1 \mathrm{H}), 8.79$ $(\mathrm{d}, J=5.8 \mathrm{~Hz}, 1 \mathrm{H}), 8.39$ (dd, $J=8.1,6.4 \mathrm{~Hz}, 1 \mathrm{H}), 7.48(\mathrm{t}, J=7.9$ $\mathrm{Hz}, 1 \mathrm{H}), 7.36$ (ddd, $J=7.7,1.9,1.0 \mathrm{~Hz}, 1 \mathrm{H}), 7.31(\mathrm{t}, J=2.1 \mathrm{~Hz}$, $1 \mathrm{H}), 7.21(\mathrm{~d}, J=5.8 \mathrm{~Hz}, 1 \mathrm{H}), 7.06(\mathrm{ddd}, J=8.2,2.4,0.9 \mathrm{~Hz}, 1 \mathrm{H})$, 5.42-5.45 (m, 1H), 2.25-2.07 (m, 2H), 1.86-1.92 (m, 2H), $1.67-1.78(\mathrm{~m}, 3 \mathrm{H}), 1.64-1.52(\mathrm{~m}, 2 \mathrm{H}), 1.44-1.49(\mathrm{~m}, 1 \mathrm{H}) .{ }^{13} \mathrm{C}$ NMR (101 MHz, MeOD) $\delta 172.42,160.45,160.12,148.65$, $142.60,140.21,139.72,136.02,132.14,129.17,119.49,118.62$, $115.32,112.51,78.48,32.28,26.45,24.56$.

$N^{4}$-Cyclohexyl- $N^{2}$-phenylpyrimidine-2,4-diamine (4a). To a mixture of $3 \mathrm{a}(0.10 \mathrm{mmol}, 1.0$ equiv $)$ in $\mathrm{EtOH}(0.50 \mathrm{~mL}, 0.20 \mathrm{M})$ under a nitrogen atmosphere at $\mathrm{rt}$ were added aniline $(0.20$ mmol, 2.0 equiv) and a drop of $1 \mathrm{~N} \mathrm{HCl}$. The reaction mixture was heated to reflux and stirred at that temperature for $1.5 \mathrm{~h}$; it was then cooled to rt. Purification using automated flash chromatography $(\mathrm{MeOH} / \mathrm{DCM})$ was followed by evaporation, giving $4 \mathrm{a}$ as a white solid $(0.018 \mathrm{~g}, 67 \%)$. TLC $R_{f} 0.7(10 \%$ $\mathrm{MeOH} / \mathrm{DCM})$. LC-MS (ESI) $m / z: 269[\mathrm{M}+\mathrm{H}]^{+} .{ }^{1} \mathrm{H}$ NMR $\left(500 \mathrm{MHz}, \mathrm{CDCl}_{3}\right) \delta 7.90-7.72(\mathrm{~m}, 1 \mathrm{H}), 7.53(\mathrm{~d}, J=7.9 \mathrm{~Hz}$, $2 \mathrm{H}), 7.25-7.20(\mathrm{~m}, 2 \mathrm{H}), 7.19(\mathrm{br} \mathrm{s}, 1 \mathrm{H}), 6.92(\mathrm{t}, J=7.4 \mathrm{~Hz}, 1 \mathrm{H})$, $5.75(\mathrm{~d}, J=5.9 \mathrm{~Hz}, 1 \mathrm{H}), 4.71(\mathrm{br} \mathrm{s}, 1 \mathrm{H}), 3.60($ br s, $1 \mathrm{H}), 2.05-$ $1.92(\mathrm{~m}, 2 \mathrm{H}), 1.69-1.75(\mathrm{~m}, 2 \mathrm{H}), 1.58-1.62(\mathrm{~m}, 1 \mathrm{H}), 1.29-$ $1.38(\mathrm{~m}, 2 \mathrm{H}), 1.21-1.08(\mathrm{~m}, 3 \mathrm{H}) .{ }^{13} \mathrm{C} \mathrm{NMR}\left(126 \mathrm{MHz} \mathrm{CDCl}_{3}\right)$ $\delta 162.03,159.44,139.97,128.73,121.97,119.23,50.07,33.09$, 25.62, 24.93 .

$N^{2}-\left(\left[1,1^{\prime}-\right.\right.$ Biphenyl]-2-yl)- $N^{4}$-cyclohexylpyrimidine-2,4-diamine (4b). To a mixture of $3 a(0.10 \mathrm{mmol}, 1.0$ equiv) in EtOH $(0.50 \mathrm{~mL}, 0.20 \mathrm{M})$ under a nitrogen atmosphere at rt were added 2 -aminobiphenyl ( $0.20 \mathrm{mmol}, 2.0$ equiv) and a drop of $1 \mathrm{~N} \mathrm{HCl}$. The reaction mixture was heated to reflux and stirred at that temperature for $1.5 \mathrm{~h}$; it was then cooled to rt. Purification using automated flash chromatography $(\mathrm{MeOH} / \mathrm{DCM})$ was followed by evaporation, giving $4 \mathrm{~b}$ as a colorless oil (0.023 g, 67\%). TLC $R_{f} 0.45$ (5\% MeOH/DCM). LC-MS (ESI) $m / z: 345[\mathrm{M}+\mathrm{H}]^{+}$. ${ }^{1} \mathrm{H}$ NMR $\left(400 \mathrm{MHz}, \mathrm{CDCl}_{3}\right) \delta 8.35(\mathrm{~d}, J=8.3 \mathrm{~Hz}, 1 \mathrm{H}), 7.78(\mathrm{~s}$, $1 \mathrm{H}), 7.51-7.39(\mathrm{~m}, 4 \mathrm{H}), 7.32-7.37(\mathrm{~m}, 2 \mathrm{H}), 7.29-7.20(\mathrm{~m}$, $2 \mathrm{H}), 7.09(\mathrm{td}, J=7.5,1.2 \mathrm{~Hz}, 1 \mathrm{H}), 5.81(\mathrm{~d}, J=6.0 \mathrm{~Hz}, 1 \mathrm{H}), 4.97$ (br s, $1 \mathrm{H}), 3.60$ (br s, $1 \mathrm{H}), 1.98-2.04(\mathrm{~m}, 2 \mathrm{H}), 1.74-1.80(\mathrm{~m}$, $2 \mathrm{H}), 1.63-1.68(\mathrm{~m}, 1 \mathrm{H}), 1.44-1.29(\mathrm{~m}, 2 \mathrm{H}), 1.15-1.27(\mathrm{~m}$, $3 \mathrm{H}) .{ }^{13} \mathrm{C}$ NMR $\left(101 \mathrm{MHz}, \mathrm{CDCl}_{3}\right) \delta 161.95,138.81,136.42$, $132.54,130.24,129.40,128.92,127.85,127.59,122.62,121.18$, $50.14,33.00,25.56,24.87$.

$N^{4}$-Cyclohexyl- $N^{2}$-(3-cyclopropylphenyl)pyrimidine-2,4-diamine (4c). To a mixture of $3 \mathrm{a}(0.10 \mathrm{mmol}, 1.0$ equiv $)$ in $\mathrm{EtOH}$ $(0.50 \mathrm{~mL}, 0.20 \mathrm{M})$ under a nitrogen atmosphere at $\mathrm{rt}$ were added 3-cyclopropylaniline $(0.11 \mathrm{mmol}, 1.1$ equiv) and a drop of $1 \mathrm{~N}$ $\mathrm{HCl}$. The reaction mixture was heated to reflux and stirred at that temperature for $4 \mathrm{~h}$; it was then cooled to rt. Purification using automated flash chromatography $(\mathrm{MeOH} / \mathrm{DCM})$ was followed by evaporation, giving $4 \mathrm{c}$ as a white oil $(0.009 \mathrm{~g}, 29 \%)$. TLC $R_{f}$ $0.6(10 \% \mathrm{MeOH} / \mathrm{DCM})$. LC-MS (ESI) $\mathrm{m} / z: 309[\mathrm{M}+\mathrm{H}]^{+} .{ }^{1} \mathrm{H}$ $\mathrm{NMR}\left(500 \mathrm{MHz}, \mathrm{CDCl}_{3}\right) \delta 7.76(\mathrm{~d}, J=6.2 \mathrm{~Hz}, 1 \mathrm{H}), 7.35-7.37$ $(\mathrm{m}, 2 \mathrm{H}), 7.20(\mathrm{~s}, 1 \mathrm{H}), 7.10(\mathrm{t}, J=7.9 \mathrm{~Hz}, 1 \mathrm{H}), 6.63(\mathrm{~d}, J=5.0 \mathrm{~Hz}$, $1 \mathrm{H}), 5.76(\mathrm{~d}, J=6.0 \mathrm{~Hz}, 1 \mathrm{H}), 4.88(\mathrm{~d}, J=8.0 \mathrm{~Hz}, 1 \mathrm{H}), 3.77-3.46$ $(\mathrm{m}, 1 \mathrm{H}), 1.95-2.00(\mathrm{~m}, 2 \mathrm{H}), 1.80-1.83(\mathrm{~m}, 1 \mathrm{H}), 1.70(\mathrm{dt}, J=$ $13.6,3.9 \mathrm{~Hz}, 2 \mathrm{H}), 1.59(\mathrm{dt}, J=13.0,3.8 \mathrm{~Hz}, 1 \mathrm{H}), 1.40-1.24(\mathrm{~m}$, 
$2 \mathrm{H}), 1.24-1.09(\mathrm{~m}, 3 \mathrm{H}), 0.91-0.79(\mathrm{~m}, 2 \mathrm{H}), 0.70-0.56(\mathrm{~m}$, 2H). ${ }^{13} \mathrm{C} \mathrm{NMR}\left(126 \mathrm{MHz}, \mathrm{CDCl}_{3}\right) \delta 162.02,158.97,153.51$, $144.68,139.65,128.60,119.35,117.17,116.73,96.62,49.98$, $33.06,25.58,24.88,15.58,9.15$.

$N^{4}$-Cyclohexyl- $N^{2}$-(3-fluorophenyl)pyrimidine-2,4-diamine (4d). To a mixture of $3 \mathrm{a}(0.10 \mathrm{mmol}, 1.0$ equiv) in $\mathrm{EtOH}(0.50$ $\mathrm{mL}, 0.20 \mathrm{M})$ at rt were added 3-fluoroaniline $(0.20 \mathrm{mmol}, 2.0$ equiv) and a drop of $1 \mathrm{~N} \mathrm{HCl}$. The reaction mixture was heated to reflux and stirred at that temperature for $1.5 \mathrm{~h}$; it was then cooled to rt. Purification using automated flash chromatography $(\mathrm{MeOH} / \mathrm{DCM})$ was followed by evaporation, giving $\mathbf{4 d}$ as a white solid (0.018 g, 63\%). TLC $R_{f} 0.7$ (10\% MeOH/DCM). LC-MS (ESI) $m / z: 287[\mathrm{M}+\mathrm{H}]^{+}$. ${ }^{1} \mathrm{H}$ NMR (500 MHz, $\left.\mathrm{CDCl}_{3}\right) \delta 7.90(\mathrm{~d}, J=6.0 \mathrm{~Hz}, 1 \mathrm{H}), 7.78(\mathrm{~d}, J=12.0 \mathrm{~Hz}, 1 \mathrm{H}), 7.30$ $(\mathrm{s}, 1 \mathrm{H}), 7.19(\mathrm{dd}, J=8.2,6.7 \mathrm{~Hz}, 1 \mathrm{H}), 7.07(\mathrm{dd}, J=8.1,2.1 \mathrm{~Hz}$, $1 \mathrm{H}), 6.66(\mathrm{td}, J=8.3,2.5 \mathrm{~Hz}, 1 \mathrm{H}), 5.85(\mathrm{~d}, J=5.8 \mathrm{~Hz}, 1 \mathrm{H}), 4.76$ (br s, $1 \mathrm{H}), 3.72($ br s, $1 \mathrm{H}), 2.16-2.00(\mathrm{~m}, 2 \mathrm{H}), 1.77-1.81(\mathrm{~m}$, $2 \mathrm{H}), 1.66-1.70(\mathrm{~m}, 1 \mathrm{H}), 1.50-1.35(\mathrm{~m}, 2 \mathrm{H}), 1.19-1.27(\mathrm{~m}$, $3 \mathrm{H}) .{ }^{13} \mathrm{C} \mathrm{NMR}\left(126 \mathrm{MHz}, \mathrm{CDCl}_{3}\right) \delta 164.17,162.25,161.98$, $159.34,141.88,141.79,129.65,129.57,114.11,114.08,108.21$, $108.04,106.15,105.93,50.12,33.11,25.62,24.90$.

$N^{4}$-Cyclohexyl- $N^{2}$-(pyridin-3-yl)pyrimidine-2,4-diamine (4e). To a mixture of $3 \mathrm{a}(0.10 \mathrm{mmol}, 1.0$ equiv) in $\mathrm{EtOH}(0.50$ $\mathrm{mL}, 0.20 \mathrm{M})$ under a nitrogen atmosphere at rt were added 3aminopyridine ( $0.20 \mathrm{mmol}, 2.0$ equiv) and a drop of $1 \mathrm{~N} \mathrm{HCl}$. The reaction mixture was heated to reflux and stirred at that temperature for $1.5 \mathrm{~h}$; it was then cooled to rt. Purification using automated flash chromatography $(\mathrm{MeOH} / \mathrm{DCM})$ was followed by evaporation, giving $4 \mathrm{e}$ as a yellow oil $(0.023 \mathrm{~g}, 85 \%)$. TLC $R_{f}$ $0.4(10 \% \mathrm{MeOH} / \mathrm{DCM})$. LC-MS (ESI) $\mathrm{m} / z: 270[\mathrm{M}+\mathrm{H}]^{+} .{ }^{1} \mathrm{H}$ NMR $(400 \mathrm{MHz}, \mathrm{MeOD}) \delta 9.29(\mathrm{~d}, J=2.3 \mathrm{~Hz}, 1 \mathrm{H}), 9.17(\mathrm{dt}, J=$ $5.1,1.7 \mathrm{~Hz}, 1 \mathrm{H}), 8.17(\mathrm{~d}, J=6.0 \mathrm{~Hz}, 1 \mathrm{H}), 7.95-7.76(\mathrm{~m}, 2 \mathrm{H})$, $6.67(\mathrm{~d}, J=6.0 \mathrm{~Hz}, 1 \mathrm{H}), 4.22-3.98(\mathrm{~m}, 1 \mathrm{H}), 2.11-1.96(\mathrm{~m}, 2 \mathrm{H})$, $1.82-1.87(\mathrm{~m}, 2 \mathrm{H}), 1.78-1.66(\mathrm{~m}, 1 \mathrm{H}), 1.48-1.55(\mathrm{~m}, 2 \mathrm{H})$, 1.44-1.19 (m, 3H). ${ }^{13} \mathrm{C}$ NMR $\left(126 \mathrm{MHz}, \mathrm{CDCl}_{3}\right) \delta 166.85$, $159.11,158.07,152.94,134.51,131.01,128.10,111.57,53.28$, $35.98,29.22,28.41$.

Methyl 3-((4-(cyclohexylamino)pyrimidin-2-yl)amino)benzoate (4f). To a mixture of $3 a(0.10 \mathrm{mmol}, 1.0$ equiv) in $\mathrm{MeOH}(0.50 \mathrm{~mL}, 0.20 \mathrm{M})$ under a nitrogen atmosphere at $\mathrm{rt}$ were added 3 -aminobenzoic acid methyl ester $(0.11 \mathrm{mmol}, 1.1$ equiv) and a drop of $1 \mathrm{~N} \mathrm{HCl}$. The reaction mixture was heated to reflux and stirred at that temperature for $2 \mathrm{~h}$; it was then cooled to rt. Purification using automated flash chromatography $(\mathrm{MeOH} / \mathrm{DCM})$ was followed by evaporation, giving $\mathbf{4 f}$ as a colorless oil $(0.018 \mathrm{~g}, 55 \%)$. TLC $R_{f} 0.6(10 \% \mathrm{MeOH} / \mathrm{DCM})$. LC-MS (ESI) $m / z$ : $327[\mathrm{M}+\mathrm{H}]^{+}$. ${ }^{1} \mathrm{H}$ NMR $(500 \mathrm{MHz}$, $\left.\mathrm{CDCl}_{3}\right) \delta 8.21(\mathrm{~s}, 1 \mathrm{H}), 7.98-7.82(\mathrm{~m}, 2 \mathrm{H}), 7.66(\mathrm{~d}, J=5.0 \mathrm{~Hz}$, $1 \mathrm{H}), 7.61(\mathrm{~s}, 1 \mathrm{H}), 7.35(\mathrm{t}, J=7.9 \mathrm{~Hz}, 1 \mathrm{H}), 5.86(\mathrm{~d}, J=5.9 \mathrm{~Hz}$, $1 \mathrm{H}), 4.88(\mathrm{br} \mathrm{s}, 1 \mathrm{H}), 3.91(\mathrm{~s}, 3 \mathrm{H}), 3.08(\mathrm{br} \mathrm{s}, 1 \mathrm{H}), 2.11-1.96(\mathrm{~m}$, $2 \mathrm{H}), 1.76(\mathrm{dt}, J=13.7,3.9 \mathrm{~Hz}, 2 \mathrm{H}), 1.70-1.60(\mathrm{~m}, 1 \mathrm{H}), 1.47-$ $1.31(\mathrm{~m}, 2 \mathrm{H}), 1.19-1.25(\mathrm{~m}, 3 \mathrm{H}) .{ }^{13} \mathrm{C} \mathrm{NMR}\left(126 \mathrm{MHz}, \mathrm{CDCl}_{3}\right)$ $\delta 167.13,162.02,159.21,154.87,140.20,130.65,128.74,123.60$, 123.02, 120.18, 52.12, 49.94, 33.05, 25.60, 24.80 .

$N^{2}$-([1,1'-Biphenyl]-3-yl)- $N^{4}$-cyclohexylpyrimidine-2,4-diamine $(\mathbf{4 g})$. To a mixture of $3 \mathrm{a}(0.10 \mathrm{mmol}, 1.0$ equiv $)$ in $\mathrm{EtOH}$ $(0.50 \mathrm{~mL}, 0.20 \mathrm{M})$ under a nitrogen atmosphere at rt were added 3-aminobiphenyl ( $0.20 \mathrm{mmol}, 2.0$ equiv) and a drop of $1 \mathrm{~N} \mathrm{HCl}$. The reaction mixture was heated to reflux and stirred at that temperature for $1.5 \mathrm{~h}$; it was then cooled to rt. Purification using automated flash chromatography $(\mathrm{MeOH} / \mathrm{DCM})$ was followed by evaporation, giving $4 \mathrm{~g}$ as a white solid $(0.018 \mathrm{~g}, 52 \%)$. TLC $R_{f}$ 0.5 (5\% MeOH/DCM). LC-MS (ESI) $m / z: 345[\mathrm{M}+\mathrm{H}]^{+} .{ }^{1} \mathrm{H}$
NMR (400 MHz, $\left.\mathrm{CDCl}_{3}\right) \delta 7.84(\mathrm{~s}, 2 \mathrm{H}), 7.59-7.62(\mathrm{~m}, 3 \mathrm{H})$, $7.43(\mathrm{dd}, J=8.2,6.9 \mathrm{~Hz}, 2 \mathrm{H}), 7.37-7.31(\mathrm{~m}, 2 \mathrm{H}), 7.23(\mathrm{dt}, J=$ $7.7,1.4 \mathrm{~Hz}, 1 \mathrm{H}), 5.86(\mathrm{~d}, J=6.0 \mathrm{~Hz}, 1 \mathrm{H}), 4.97(\mathrm{~d}, J=8.0 \mathrm{~Hz}, 1 \mathrm{H})$, $3.71(\mathrm{~s}, 1 \mathrm{H}), 2.10-1.92(\mathrm{~m}, 2 \mathrm{H}), 1.77-1.64(\mathrm{~m}, 2 \mathrm{H}), 1.59(\mathrm{~d}, J=$ $11.9 \mathrm{~Hz}, 1 \mathrm{H}), 1.36-1.09(\mathrm{~m}, 5 \mathrm{H}) .{ }^{13} \mathrm{C} \mathrm{NMR}\left(101 \mathrm{MHz}, \mathrm{CDCl}_{3}\right)$ $\delta 162.02,142.00,141.39,140.04,129.07,128.67,127.28,121.29$, 118.42, 50.06, 33.02, 25.53, 24.73.

$N^{4}$-Cyclohexyl- $N^{2}$-(3-(pyridin-2-yl)phenyl)pyrimidine-2,4diamine (4h). To a mixture of $3 \mathrm{a}(0.10 \mathrm{mmol}, 1.0$ equiv) in $\mathrm{EtOH}(0.50 \mathrm{~mL}, 0.20 \mathrm{M})$ under a nitrogen atmosphere at rt were added 3-(pyridin-2-yl)aniline (0.11 mmol, 1.1 equiv) and a drop of $1 \mathrm{~N} \mathrm{HCl}$. The reaction mixture was heated to reflux and stirred at that temperature for $3 \mathrm{~h}$; it was then cooled to rt. Purification using automated flash chromatography ( $\mathrm{MeOH} / \mathrm{DCM})$ was followed by evaporation, giving $4 \mathrm{~h}$ as a white film $(0.007 \mathrm{~g}, 20 \%)$. TLC $R_{f} 0.5$ (10\% MeOH/DCM). LC-MS (ESI) $m / z: 346[\mathrm{M}+$ $\mathrm{H}]^{+} .{ }^{1} \mathrm{H} \mathrm{NMR}(500 \mathrm{MHz}, \mathrm{MeOD}) \delta 8.74-8.58(\mathrm{~m}, 1 \mathrm{H}), 8.27(\mathrm{~s}$, $1 \mathrm{H}), 7.95(\mathrm{td}, J=7.7,1.8 \mathrm{~Hz}, 1 \mathrm{H}), 7.89-7.94(\mathrm{~m}, 1 \mathrm{H}), 7.73(\mathrm{~d}, J$ $=7.4 \mathrm{~Hz}, 1 \mathrm{H}), 7.70-7.63(\mathrm{~m}, 1 \mathrm{H}), 7.60-7.55(\mathrm{~m}, 1 \mathrm{H}), 7.52(\mathrm{t}, J$ $=7.8 \mathrm{~Hz}, 1 \mathrm{H}), 7.41-7.43(\mathrm{~m}, 1 \mathrm{H}), 6.14(\mathrm{~d}, J=7.0 \mathrm{~Hz}, 1 \mathrm{H}), 3.90$ (s, $1 \mathrm{H}), 2.06-1.89(\mathrm{~m}, 2 \mathrm{H}), 1.74-1.60(\mathrm{~m}, 2 \mathrm{H}), 1.60-1.49(\mathrm{~m}$, $1 \mathrm{H}), 1.30-1.21(\mathrm{~m}, 2 \mathrm{H}), 1.21-1.05(\mathrm{~m}, 3 \mathrm{H}) .{ }^{13} \mathrm{C}$ NMR $(126$ $\mathrm{MHz}, \mathrm{MeOD}) \delta 161.97,157.24,154.20,149.00,143.49,140.02$, $138.14,137.66,122.94,122.63,122.01,121.48,120.39,98.62$, $50.18,31.86,25.07,24.52$.

$N^{4}$-Cyclohexyl-N2-(3'-methoxy-[1,1'-biphenyl]-3-yl)pyrimidine-2,4-diamine (4i). To a mixture of $3 \mathrm{a}(0.10 \mathrm{mmol}, 1.0$ equiv) in $\mathrm{EtOH}(0.50 \mathrm{~mL}, 0.20 \mathrm{M})$ under a nitrogen atmosphere at $\mathrm{rt}$ were added ( $3^{\prime}$-methoxybiphenyl-3-yl)amine $\mathrm{HCl}(0.11$ mmol, 1.1 equiv) and a drop of $1 \mathrm{~N} \mathrm{HCl}$. The reaction mixture was heated to reflux and stirred at that temperature for $3 \mathrm{~h}$; it was then cooled to rt. Purification using automated flash chromatography (EtOAc/hexanes) was followed by evaporation, giving $4 \mathbf{i}$ as a colorless oil $(0.007 \mathrm{~g}, 19 \%)$. TLC $R_{f} 0.2$ (50\% EtOAc/ hexanes + 1\% MeOH). LC-MS (ESI) $m / z: 375[\mathrm{M}+\mathrm{H}]^{+} .{ }^{1} \mathrm{H}$ NMR $\left(500 \mathrm{MHz}, \mathrm{CDCl}_{3}\right) \delta 7.98-7.89(\mathrm{~m}, 1 \mathrm{H}), 7.85(\mathrm{~s}, 1 \mathrm{H})$, 7.63-7.65 (m, 1H), 7.35-7.37 (m, 2H), 7.21-7.24 (m, 2H), $7.18(\mathrm{~s}, 1 \mathrm{H}), 7.15-7.16(\mathrm{~m}, 1 \mathrm{H}), 6.90-6.93(\mathrm{~m}, 1 \mathrm{H}), 5.85(\mathrm{~d}, J=$ $5.9 \mathrm{~Hz}, 1 \mathrm{H}), 4.71(\mathrm{~s}, 1 \mathrm{H}), 3.89(\mathrm{~s}, 3 \mathrm{H}), 3.84-3.57(\mathrm{~m}, 1 \mathrm{H})$, 2.03-2.07 (m, 2H), 1.81-1.68 (m, 2H), $1.68-1.56(\mathrm{~m}, 1 \mathrm{H})$, $1.39-1.27(\mathrm{~m}, 2 \mathrm{H}), 1.17-1.25(\mathrm{~m}, 3 \mathrm{H}) .{ }^{13} \mathrm{C}$ NMR (126 MHz, $\left.\mathrm{CDCl}_{3}\right) \delta 162.06,159.85,159.82,155.82,143.05,141.85,140.49$, $129.64,129.03,120.89,119.83,118.28,118.07,112.97,112.67$, 95.81, 55.30, 49.85, 33.12, 25.61, 24.74.

Methyl 3'-((4-(cyclohexylamino)pyrimidin-2-yl)amino)$\left[1,1^{\prime}\right.$-biphenyl]-3-carboxylate (4j). To a mixture of 3a $(0.10$ mmol, 1.0 equiv $)$ in $\mathrm{EtOH}(0.50 \mathrm{~mL}, 0.20 \mathrm{M})$ under a nitrogen atmosphere at rt were added 3 '-aminobiphenyl-3-carboxylic acid methyl ester $\mathrm{HCl}(0.11 \mathrm{mmol}, 1.1$ equiv) and a drop of $1 \mathrm{~N} \mathrm{HCl}$. The reaction mixture was heated to reflux and stirred at that temperature for $3 \mathrm{~h}$; it was then cooled to rt. Purification using automated flash chromatography $(\mathrm{MeOH} / \mathrm{DCM})$ was followed by evaporation, giving $4 \mathbf{j}$ as a white solid $(0.012 \mathrm{~g}, 30 \%)$. TLC $R_{f}$ $0.2(50 \% \mathrm{EtOAc} /$ hexanes $+1 \% \mathrm{MeOH})$. LC-MS (ESI) $\mathrm{m} / z$ : $375[\mathrm{M}+\mathrm{H}]^{+} .{ }^{1} \mathrm{H} \mathrm{NMR}\left(500 \mathrm{MHz}, \mathrm{CDCl}_{3}\right) \delta 8.31(\mathrm{t}, J=1.8 \mathrm{~Hz}$, $1 \mathrm{H}), 8.04(\mathrm{dt}, J=7.8,1.4 \mathrm{~Hz}, 1 \mathrm{H}), 7.97-7.87(\mathrm{~m}, 2 \mathrm{H}), 7.82$ (ddd, $J=7.8,1.9,1.2 \mathrm{~Hz}, 1 \mathrm{H}), 7.64(\mathrm{ddd}, J=8.1,2.3,1.0 \mathrm{~Hz}, 1 \mathrm{H})$, $7.53(\mathrm{t}, J=7.7 \mathrm{~Hz}, 1 \mathrm{H}), 7.40(\mathrm{t}, J=7.9 \mathrm{~Hz}, 1 \mathrm{H}), 7.26(\mathrm{ddd}, J=$ $7.7,1.8,1.0 \mathrm{~Hz}, 1 \mathrm{H}), 7.20(\mathrm{~s}, 1 \mathrm{H}), 5.86(\mathrm{~d}, J=5.9 \mathrm{~Hz}, 1 \mathrm{H}), 4.74$ (br s, $1 \mathrm{H}), 3.97$ (br s, 3H), 3.86-3.56 (m, 1H), 2.11-1.99 (m, $2 \mathrm{H}), 1.78-1.67(\mathrm{~m}, 2 \mathrm{H}), 1.61(\mathrm{~d}, J=11.2 \mathrm{~Hz}, 1 \mathrm{H}), 1.36-1.13$ $(\mathrm{m}, 5 \mathrm{H}) .{ }^{13} \mathrm{C} \mathrm{NMR}\left(126 \mathrm{MHz}, \mathrm{CDCl}_{3}\right) \delta 167.09,162.04,159.62$, $155.39,141.74,140.83,140.59,131.68,130.59,129.22$, 128.77, 
$128.42,128.31,120.85,118.56,117.98,77.27,52.19,49.89$, $33.08,25.56,24.75$.

$N^{4}$-Cyclohexyl- $N^{2}-(3-(o x a z o l-2-y l) p h e n y l) p y r i m i d i n e-2,4-$ diamine $(4 \mathrm{k})$. To a mixture of $3 \mathrm{a}(0.10 \mathrm{mmol}, 1.0$ equiv $)$ in $\mathrm{EtOH}(0.50 \mathrm{~mL}, 0.20 \mathrm{M})$ under a nitrogen atmosphere at rt were added 3-(1,3-oxaxol-2-yl)aniline ( $0.11 \mathrm{mmol}, 1.1$ equiv) and a drop of $1 \mathrm{~N} \mathrm{HCl}$. The reaction mixture was heated to reflux and stirred at that temperature for $2 \mathrm{~h}$; it was then cooled to rt. Purification using automated flash chromatography $(\mathrm{MeOH} /$ DCM) was followed by evaporation, giving $4 \mathbf{k}$ as a white solid (0.022 g, 66\%). TLC $R_{f} 0.6$ (10\% MeOH/DCM). LC-MS (ESI) $m / z: 336[\mathrm{M}+\mathrm{H}]^{+} .{ }^{1} \mathrm{H} \mathrm{NMR}\left(500 \mathrm{MHz}, \mathrm{CDCl}_{3}\right) \delta 8.40(\mathrm{~s}, 1 \mathrm{H})$, 7.97-7.83 (m, 1H), 7.76-7.65 (m, 3H), $7.41(\mathrm{~s}, 1 \mathrm{H}), 7.28(\mathrm{~s}$, $1 \mathrm{H}), 7.25(\mathrm{~s}, 1 \mathrm{H}), 5.89(\mathrm{~d}, J=6.0 \mathrm{~Hz}, 1 \mathrm{H}), 5.04$ (br s, $1 \mathrm{H}), 3.74$ (br s, $1 \mathrm{H}), 2.13-2.01(\mathrm{~m}, 2 \mathrm{H}), 1.76-1.80(\mathrm{~m}, 2 \mathrm{H}), 1.65-1.69$ $(\mathrm{m}, 1 \mathrm{H}), 1.47-1.34(\mathrm{~m}, 2 \mathrm{H}), 1.21-1.29(\mathrm{~m}, 3 \mathrm{H}) .{ }^{13} \mathrm{C} \mathrm{NMR}$ $\left(126 \mathrm{MHz}, \mathrm{CDCl}_{3}\right) \delta 162.08,162.02,158.70,140.30,138.48$, $129.23,128.37,127.92,121.20,120.13,117.01,50.03,33.08$, 25.57, 24.81 .

$N^{2}$-(3-(1H-Pyrazol-3-yl)phenyl)- $N^{4}$-cyclohexylpyrimidine2,4-diamine (4l). To a mixture of $3 \mathrm{a}(0.10 \mathrm{mmol}, 1.0$ equiv) in EtOH $(0.50 \mathrm{~mL}, 0.20 \mathrm{M})$ under a nitrogen atmosphere at rt were added 3-(1H-pyrazol-3-yl)aniline $(0.11 \mathrm{mmol}, 1.1$ equiv $)$ and a drop of $1 \mathrm{~N} \mathrm{HCl}$. The reaction mixture was heated to reflux and stirred at that temperature for $2 \mathrm{~h}$; it was then cooled to rt. Purification using automated flash chromatography $(\mathrm{MeOH} /$ DCM) was followed by evaporation, giving $\mathbf{4 l}$ as a colorless oil (0.020 g, 60\%). TLC $R_{f} 0.6$ (10\% MeOH/DCM). LC-MS (ESI) $m / z: 335[\mathrm{M}+\mathrm{H}]^{+} .{ }^{1} \mathrm{H} \mathrm{NMR}(500 \mathrm{MHz}, \mathrm{MeOD}) \delta 8.08(\mathrm{~s}, 1 \mathrm{H})$, $7.69(\mathrm{~d}, J=16.6 \mathrm{~Hz}, 2 \mathrm{H}), 7.56(\mathrm{~d}, J=8.0 \mathrm{~Hz}, 1 \mathrm{H}), 7.43(\mathrm{~d}, J=8.0$ $\mathrm{Hz}, 1 \mathrm{H}), 7.36(\mathrm{td}, J=7.8,1.8 \mathrm{~Hz}, 1 \mathrm{H}), 6.67(\mathrm{~d}, J=2.5 \mathrm{~Hz}, 1 \mathrm{H})$, $5.99(\mathrm{dd}, J=6.4,1.8 \mathrm{~Hz}, 1 \mathrm{H}), 3.92(\mathrm{~s}, 1 \mathrm{H}), 2.00-2.02(\mathrm{~m}, 2 \mathrm{H})$, $1.72-1.75(\mathrm{~m}, 2 \mathrm{H}), 1.61-1.63(\mathrm{~m}, 1 \mathrm{H}), 1.19-1.31(\mathrm{~m}, 5 \mathrm{H}) .{ }^{13} \mathrm{C}$ NMR (126 MHz, MeOD) $\delta 162.23,158.06,150.32,140.11$, $128.66,119.78,119.48,117.56,102.17,97.88,49.48,32.36$, $25.35,24.70$.

$N^{2}$-(3-(1H-Imidazol-1-yl)phenyl)- $N^{4}$-cyclohexylpyrimidine2,4-diamine $(4 \mathrm{~m})$. To a mixture of $3 \mathrm{a}(0.10 \mathrm{mmol}, 1.0$ equiv $)$ in EtOH $(0.50 \mathrm{~mL}, 0.20 \mathrm{M})$ under a nitrogen atmosphere at rt were added 3-( $1 \mathrm{H}$-imidazol-1-yl)aniline $(0.11 \mathrm{mmol}, 1.1$ equiv $)$ and a drop of $1 \mathrm{~N} \mathrm{HCl}$. The reaction mixture was heated to reflux and stirred at that temperature for $20 \mathrm{~h}$; it was then cooled to rt. Purification using automated reversed-phase flash chromatography (water $/ \mathrm{MeOH}$ ) was followed by evaporation, giving $4 \mathrm{~m}$ as a colorless oil $(0.010 \mathrm{~g}, 30 \%)$. LC-MS (ESI) $m / z: 335[\mathrm{M}+\mathrm{H}]^{+}$. ${ }^{1} \mathrm{H}$ NMR (400 MHz, MeOD) $\delta 8.50$ (br s, $\left.1 \mathrm{H}\right), 8.46$ (d, $J=2.2$ $\mathrm{Hz}, 1 \mathrm{H}), 8.16-8.01(\mathrm{~m}, 2 \mathrm{H}), 7.35(\mathrm{t}, J=8.0 \mathrm{~Hz}, 1 \mathrm{H}), 7.06-6.95$ $(\mathrm{m}, 2 \mathrm{H}), 6.91(\mathrm{ddd}, J=8.2,2.1,0.9 \mathrm{~Hz}, 1 \mathrm{H}), 6.56(\mathrm{~d}, J=6.1 \mathrm{~Hz}$, $1 \mathrm{H}), 4.13-4.14(\mathrm{~m}, 1 \mathrm{H}), 2.11-1.97(\mathrm{~m}, 2 \mathrm{H}), 1.81-1.86(\mathrm{~m}$, $2 \mathrm{H}), 1.70-1.74(\mathrm{~m}, 1 \mathrm{H}), 1.57-1.44(\mathrm{~m}, 2 \mathrm{H}), 1.36-1.39(\mathrm{~m}$, $3 \mathrm{H}) .{ }^{13} \mathrm{C}$ NMR (101 MHz, MeOD) $\delta 164.23,155.53,153.70$, $151.78,137.14,131.98,123.60,120.79,117.54,111.20,108.63$, $107.95,50.50,33.57,26.75,25.94$.

$N^{4}$-Cyclohexyl- $N^{2}-(3-($ pyrazin-2-yl)phenyl)pyrimidine-2,4diamine (4n). To a mixture of $3 \mathrm{a}(0.10 \mathrm{mmol}, 1.0$ equiv $)$ in $\mathrm{EtOH}(0.50 \mathrm{~mL}, 0.20 \mathrm{M})$ under a nitrogen atmosphere at rt were added 3-(pyrazin-2-yl)aniline ( $0.11 \mathrm{mmol}, 1.1$ equiv) and a drop of $1 \mathrm{~N} \mathrm{HCl}$. The reaction mixture was heated to reflux and stirred at that temperature for $5 \mathrm{~h}$; it was then cooled to rt. Purification using automated flash chromatography ( $\mathrm{MeOH} / \mathrm{EtOAc})$ was followed by evaporation, giving $\mathbf{4 n}$ as a colorless oil $(0.007 \mathrm{~g}$, $20 \%$ ). TLC $R_{f} 0.7$ (10\% MeOH/EtOAc). LC-MS (ESI) $m / z$ : $347[\mathrm{M}+\mathrm{H}]^{+} .{ }^{1} \mathrm{H}$ NMR (400 MHz, MeOD) $\delta 9.13$ (d, $J=1.6$
$\mathrm{Hz}, 1 \mathrm{H}), 8.75-8.67(\mathrm{~m}, 1 \mathrm{H}), 8.57(\mathrm{~d}, J=2.5 \mathrm{~Hz}, 1 \mathrm{H}), 8.43$ (s, $1 \mathrm{H}), 7.72(\mathrm{t}, J=8.7 \mathrm{~Hz}, 3 \mathrm{H}), 7.49(\mathrm{t}, J=7.9 \mathrm{~Hz}, 1 \mathrm{H}), 6.02(\mathrm{~d}, J=$ $6.3 \mathrm{~Hz}, 1 \mathrm{H}), 3.91(\mathrm{~s}, 1 \mathrm{H}), 1.99-2.03(\mathrm{~m}, 2 \mathrm{H}), 1.70-1.72(\mathrm{~m}$, $2 \mathrm{H}), 1.60(\mathrm{~s}, 1 \mathrm{H}), 1.37-1.13(\mathrm{~m}, 5 \mathrm{H}) .{ }^{13} \mathrm{C}$ NMR $(101 \mathrm{MHz}$, MeOD) $\delta 163.62,158.67,154.55,145.64,144.08,143.40$, 141.62 , 138.07, 130.47, 123.02, 122.50, 120.22, 101.40, 51.02, $33.61,26.67,26.06$.

$N^{4}$-Cyclohexyl- $N^{2}$-(3-morpholinophenyl)pyrimidine-2,4-diamine (4o). To a mixture of $3 \mathrm{a}(0.10 \mathrm{mmol}, 1.0$ equiv) in $\mathrm{EtOH}$ $(0.50 \mathrm{~mL}, 0.20 \mathrm{M})$ under a nitrogen atmosphere at $\mathrm{rt}$ were added 3-morpholin-4-ylaniline ( $0.11 \mathrm{mmol}, 1.1$ equiv) and a drop of 1 $\mathrm{N} \mathrm{HCl}$. The reaction mixture was heated to reflux and stirred at that temperature for $3 \mathrm{~h}$; it was then cooled to rt. Purification using automated flash chromatography ( $\mathrm{MeOH} / \mathrm{DCM})$ was followed by evaporation, giving 40 as a white oil $(0.018 \mathrm{~g}, 51 \%)$. TLC $R_{f} 0.4$ (5\% MeOH/DCM). LC-MS (ESI) $m / z: 354[\mathrm{M}+$ $\mathrm{H}]^{+}$. ${ }^{1} \mathrm{H}$ NMR $\left(400 \mathrm{MHz}, \mathrm{CDCl}_{3}\right) \delta 7.62(\mathrm{~s}, 1 \mathrm{H}), 7.21(\mathrm{~d}, J=6.6$ $\mathrm{Hz}, 3 \mathrm{H}), 7.11-7.02(\mathrm{~m}, 1 \mathrm{H}), 6.64(\mathrm{dd}, J=5.7,3.4 \mathrm{~Hz}, 1 \mathrm{H}), 5.98$ $(\mathrm{d}, J=6.7 \mathrm{~Hz}, 1 \mathrm{H}), 3.91-3.70(\mathrm{~m}, 5 \mathrm{H}), 3.22-3.10(\mathrm{~m}, 4 \mathrm{H})$, $2.10-1.93(\mathrm{~m}, 2 \mathrm{H}), 1.76-1.82(\mathrm{~m}, 2 \mathrm{H}), 1.64-1.69(\mathrm{~m}, 1 \mathrm{H})$, 1.46-1.12 (m, 5H). ${ }^{13} \mathrm{C}$ NMR (101 MHz, $\left.\mathrm{CDCl}_{3}\right) \delta 161.86$, $151.96,138.95,129.33,112.24,111.16,107.93,66.89,50.64$, 49.30, 32.63, 25.36, 24.77.

$N^{4}$-Cyclohexyl- $N^{2}$-(1-phenylpiperidin-3-yl)pyrimidine-2,4diamine (4p). To a mixture of $3 \mathrm{a}(0.10 \mathrm{mmol}, 1.0$ equiv) in $\mathrm{EtOH}(0.50 \mathrm{~mL}, 0.20 \mathrm{M})$ under a nitrogen atmosphere at rt were added 1-phenylpiperidin-3-amine $(0.11 \mathrm{mmol}, 1.1$ equiv) and a drop of $1 \mathrm{~N} \mathrm{HCl}$. The reaction mixture was heated to reflux and stirred at that temperature for $22 \mathrm{~h}$; it was then cooled to rt. Purification using automated flash chromatography ( $\mathrm{MeOH} /$ EtOAc) was followed by evaporation, giving $4 \mathrm{p}$ as a colorless oil (0.002 g, 6\%). TLC $R_{f} 0.3$ (10\% MeOH/EtOAc). LC-MS (ESI) $m / z: 352[\mathrm{M}+\mathrm{H}]^{+} .{ }^{1} \mathrm{H}$ NMR $(500 \mathrm{MHz}, \mathrm{MeOD}) \delta 7.58(\mathrm{~s}, 1 \mathrm{H})$, $7.23(\mathrm{t}, J=10.0 \mathrm{~Hz}, 2 \mathrm{H}), 7.00(\mathrm{~d}, J=8.1 \mathrm{~Hz}, 2 \mathrm{H}), 6.83(\mathrm{t}, J=7.3$ $\mathrm{Hz}, 1 \mathrm{H}), 5.84(\mathrm{~d}, J=6.5 \mathrm{~Hz}, 1 \mathrm{H}), 4.17-4.03(\mathrm{~m}, 1 \mathrm{H}), 3.87(\mathrm{~s}$, $1 \mathrm{H}), 3.70(\mathrm{~d}, J=11.6 \mathrm{~Hz}, 1 \mathrm{H}), 3.44-3.36(\mathrm{~m}, 1 \mathrm{H}), 2.93(\mathrm{t}, J=$ $15.0 \mathrm{~Hz}, 1 \mathrm{H}), 2.82-2.73(\mathrm{~m}, 1 \mathrm{H}), 1.98(\mathrm{~d}, J=8.1 \mathrm{~Hz}, 3 \mathrm{H}), 1.96-$ $1.86(\mathrm{~m}, 1 \mathrm{H}), 1.79-1.71(\mathrm{~m}, 2 \mathrm{H}), 1.58-1.65(\mathrm{~m}, 2 \mathrm{H}), 1.31-$ $1.40(\mathrm{~m}, 2 \mathrm{H}), 1.20-1.29(\mathrm{~m}, 3 \mathrm{H}) .{ }^{13} \mathrm{C}$ NMR $(126 \mathrm{MHz}$, MeOD) $\delta 162.32,151.98,128.59,119.61,116.99,55.38,50.15$, 49.16, 32.45, 32.40, 29.61, 25.36, 24.67, 23.24.

$N^{4}$-Cyclohexyl- $N^{2}$-(3-(pyridin-3-yl)cyclohexyl)pyrimidine2,4-diamine (4q). To a mixture of $3 \mathrm{a}(0.080 \mathrm{mmol}, 1.0$ equiv $)$ in $\mathrm{EtOH}(0.50 \mathrm{~mL}, 0.16 \mathrm{M})$ under a nitrogen atmosphere at rt were added 3-(pyridin-3-yl)cyclohexan-1-amine (0.080 mmol, 1.0 equiv) and a drop of $1 \mathrm{~N} \mathrm{HCl}$. The reaction mixture was heated to reflux and stirred at that temperature for $6 \mathrm{~h}$; it was then cooled to rt. Purification using automated flash chromatography $(\mathrm{MeOH} / \mathrm{DCM})$ was followed by evaporation, giving $\mathbf{4 q}$ as a yellow oil (0.002 g, 7\%). TLC $R_{f} 0.2(10 \% \mathrm{MeOH} / \mathrm{DCM})$. LCMS (ESI) $m / z: 353[\mathrm{M}+\mathrm{H}]^{+} .{ }^{1} \mathrm{H}$ NMR (500 MHz, MeOD) $\delta$ $9.91(\mathrm{~d}, J=7.0 \mathrm{~Hz}, 2 \mathrm{H}), 8.79(\mathrm{~d}, J=8.0 \mathrm{~Hz}, 1 \mathrm{H}), 8.22(\mathrm{q}, J=5.0$ $\mathrm{Hz}, 2 \mathrm{H}), 6.70(\mathrm{~d}, J=6.0 \mathrm{~Hz}, 1 \mathrm{H}), 4.18-4.04(\mathrm{~m}, 1 \mathrm{H}), 3.78(\mathrm{td}, J$ $=10.8,5.4 \mathrm{~Hz}, 1 \mathrm{H}), 3.11(\mathrm{t}, J=12.3 \mathrm{~Hz}, 1 \mathrm{H}), 2.28(\mathrm{~d}, J=11.3 \mathrm{~Hz}$, $1 \mathrm{H}), 2.13-2.05(\mathrm{~m}, 3 \mathrm{H}), 2.05-1.96(\mathrm{~m}, 2 \mathrm{H}), 1.84-1.89(\mathrm{~m}$, $2 \mathrm{H}), 1.74(\mathrm{dt}, J=12.8,4.1 \mathrm{~Hz}, 1 \mathrm{H}), 1.48-1.63(\mathrm{~m}, 4 \mathrm{H}), 1.44-$ $1.28(\mathrm{~m}, 4 \mathrm{H}) .{ }^{13} \mathrm{C}$ NMR $(126 \mathrm{MHz}, \mathrm{MeOD}) \delta 162.95,155.05$, 154.22 , 147.78, 146.83, 138.49, 138.24, 127.22, 107.83, 69.34, 49.71, 41.40, 39.97, 34.25, 32.11, 31.96, 25.32, 24.56, 23.72.

$N^{4}$-Cyclohexyl- $N^{2}$-(4-fluorophenyl)pyrimidine-2,4-diamine (4r). To a mixture of $3 \mathrm{a}(0.10 \mathrm{mmol}, 1.0$ equiv) in $\mathrm{EtOH}(0.50$ $\mathrm{mL}, 0.20 \mathrm{M})$ under a nitrogen atmosphere at rt were added 4fluoroaniline ( $0.20 \mathrm{mmol}, 2.0$ equiv) and a drop of $1 \mathrm{~N} \mathrm{HCl}$. The 
reaction mixture was heated to reflux and stirred at that temperature for $1.5 \mathrm{~h}$; it was then cooled to rt. Purification using automated flash chromatography $(\mathrm{MeOH} / \mathrm{DCM})$ was followed by evaporation, giving $4 \mathbf{r}$ as a white solid $(0.024 \mathrm{~g}, 84 \%)$. TLC $R_{f}$ $0.7(10 \% \mathrm{MeOH} / \mathrm{DCM})$. LC-MS (ESI) $m / z: 287[\mathrm{M}+\mathrm{H}]^{+} .{ }^{1} \mathrm{H}$ NMR $\left(500 \mathrm{MHz}, \mathrm{CDCl}_{3}\right) \delta 7.77(\mathrm{~s}, 1 \mathrm{H}), 7.65-7.46(\mathrm{~m}, 2 \mathrm{H})$, 7.09-6.89 (m, 2H), $5.95(\mathrm{~d}, J=6.2 \mathrm{~Hz}, 1 \mathrm{H}), 5.50(\mathrm{br} \mathrm{s}, 2 \mathrm{H}), 3.71$ (br s, $1 \mathrm{H}), 2.05$ (dd, $J=12.9,4.3 \mathrm{~Hz}, 2 \mathrm{H}), 1.78-1.83(\mathrm{~m}, 2 \mathrm{H})$, $1.67-1.71(\mathrm{~m}, 1 \mathrm{H}), 1.47-1.33(\mathrm{~m}, 2 \mathrm{H}), 1.22-1.30(\mathrm{~m}, 3 \mathrm{H}) .{ }^{13} \mathrm{C}$ NMR $\left(126 \mathrm{MHz}, \mathrm{CDCl}_{3}\right) \delta 161.93,159.61,157.69,135.07$, 121.63, 115.39, 50.47, 32.81, 25.49, 24.88.

4-((4-(Cyclohexylamino)pyrimidin-2-yl)amino)benzamide (4s). To a mixture of $3 \mathrm{a}(0.10 \mathrm{mmol}, 1.0$ equiv $)$ in $\mathrm{EtOH}(0.50$ $\mathrm{mL}, 0.20 \mathrm{M})$ under a nitrogen atmosphere at rt were added 4aminobenzamide ( $0.20 \mathrm{mmol}, 2.0$ equiv) and a drop of $1 \mathrm{~N} \mathrm{HCl}$. The reaction mixture was heated to reflux and stirred at that temperature for $2 \mathrm{~h}$; it was then cooled to rt. Purification using automated flash chromatography $(\mathrm{MeOH} / \mathrm{DCM})$ was followed by evaporation, giving $4 \mathrm{~s}$ as a white solid $(0.011 \mathrm{~g}, 35 \%)$. TLC $R_{f}$ $0.5(10 \% \mathrm{MeOH} / \mathrm{DCM})$. LC-MS (ESI) $m / z: 312[\mathrm{M}+\mathrm{H}]^{+} .{ }^{1} \mathrm{H}$ NMR (400 MHz, MeOD) $\delta 7.87-7.79(\mathrm{~m}, 4 \mathrm{H}), 7.75(\mathrm{~d}, J=6.2$ $\mathrm{Hz}, 1 \mathrm{H}), 6.00(\mathrm{~d}, J=6.1 \mathrm{~Hz}, 1 \mathrm{H}), 3.93(\mathrm{~s}, 1 \mathrm{H}), 2.15-2.00(\mathrm{~m}$, $2 \mathrm{H}), 1.83-1.88(\mathrm{~m}, 2 \mathrm{H}), 1.71-1.76(\mathrm{~m}, 1 \mathrm{H}), 1.57-1.41(\mathrm{~m}$, $2 \mathrm{H}), 1.27-1.34(\mathrm{~m}, 3 \mathrm{H}) .{ }^{13} \mathrm{C}$ NMR (101 MHz, MeOD) $\delta$ $172.05,163.65,153.57,145.07,130.50,129.60,119.66,114.60$, $51.24,33.74,26.83,26.36$.

$N^{4}$-Cyclohexyl- $N^{2}$-(4-(pyridin-2-yl)phenyl)pyrimidine-2,4diamine (4t). To a mixture of $3 \mathrm{a}(0.10 \mathrm{mmol}, 1.0$ equiv $)$ in $\mathrm{EtOH}$ $(0.50 \mathrm{~mL}, 0.20 \mathrm{M})$ under a nitrogen atmosphere at $\mathrm{rt}$ were added 4-(2-pyridyl)aniline (0.11 mmol, 1.1 equiv) and a drop of $1 \mathrm{~N}$ $\mathrm{HCl}$. The reaction mixture was heated to reflux and stirred at that temperature for $3 \mathrm{~h}$; it was then cooled to rt. Purification using automated flash chromatography (EtOAc/hexanes $+2 \% \mathrm{MeOH}$ additive) was followed by evaporation, giving $4 \mathbf{t}$ as a colorless oil $(0.002 \mathrm{~g}, 6 \%)$. TLC $R_{f} 0.2$ (50\% EtOAc/hexanes $\left.+1 \% \mathrm{MeOH}\right)$. LC-MS (ESI) $m / z: 346[\mathrm{M}+\mathrm{H}]^{+}$. ${ }^{1} \mathrm{H}$ NMR (500 MHz, $\left.\mathrm{CDCl}_{3}\right) \delta 8.59(\mathrm{~d}, J=4.8 \mathrm{~Hz}, 1 \mathrm{H}), 7.89(\mathrm{~d}, J=8.5 \mathrm{~Hz}, 2 \mathrm{H}), 7.82$ $(\mathrm{d}, J=6.2 \mathrm{~Hz}, 1 \mathrm{H}), 7.71-7.60(\mathrm{~m}, 3 \mathrm{H}), 7.42-7.25(\mathrm{~m}, 1 \mathrm{H})$, $7.16-7.19(\mathrm{~m}, 1 \mathrm{H}), 5.79(\mathrm{~d}, J=5.9 \mathrm{~Hz}, 1 \mathrm{H}), 4.76(\mathrm{~s}, 1 \mathrm{H}), 3.84-$ $3.45(\mathrm{~m}, 1 \mathrm{H}), 2.07-2.10(\mathrm{~m}, 2 \mathrm{H}), 1.67-1.79(\mathrm{~m}, 2 \mathrm{H}), 1.66-$ $1.56(\mathrm{~m}, 1 \mathrm{H}), 1.45-1.30(\mathrm{~m}, 2 \mathrm{H}), 1.21-1.29(\mathrm{~m}, 3 \mathrm{H}) .{ }^{13} \mathrm{C} \mathrm{NMR}$ $\left(126 \mathrm{MHz}, \mathrm{CDCl}_{3}\right) \delta 162.02,158.96,157.19,149.55,140.80$, $136.63,132.88,127.36,121.42,119.86,118.96,118.84,50.15$, 33.06, 33.03, 25.60, 24.93.

$N^{4}$-Cyclohexyl- $N^{2}-(4-($ pyridin-3-yl)phenyl)pyrimidine-2,4diamine $(4 u)$. To a mixture of $3 a(0.10 \mathrm{mmol}, 1.0$ equiv $)$ in $\mathrm{EtOH}(0.50 \mathrm{~mL}, 0.20 \mathrm{M})$ under a nitrogen atmosphere at rt were added 4-pyridin-3-ylaniline ( $0.11 \mathrm{mmol}, 1.1$ equiv) and a drop of $1 \mathrm{~N} \mathrm{HCl}$. The reaction mixture was heated to reflux and stirred at that temperature for $4 \mathrm{~h}$; it was then cooled to rt. Purification using automated flash chromatography $(\mathrm{MeOH} / \mathrm{DCM})$ was followed by evaporation, giving $4 \mathbf{u}$ as a colorless oil $(0.001 \mathrm{~g}$, $3 \%)$. TLC $R_{f} 0.2$ (10\% MeOH/DCM). LC-MS (ESI) $m / z: 346$ $[\mathrm{M}+\mathrm{H}]^{+} .{ }^{1} \mathrm{H}$ NMR $(500 \mathrm{MHz}, \mathrm{MeOD}) \delta 10.18-10.05(\mathrm{~m}$, $1 \mathrm{H}), 9.83(\mathrm{~d}, J=6.2 \mathrm{~Hz}, 1 \mathrm{H}), 8.96(\mathrm{dd}, J=8.5,2.1 \mathrm{~Hz}, 1 \mathrm{H})$, $8.28-8.17(\mathrm{~m}, 2 \mathrm{H}), 7.65(\mathrm{~d}, J=8.2 \mathrm{~Hz}, 2 \mathrm{H}), 6.88(\mathrm{~d}, J=8.2 \mathrm{~Hz}$, $2 \mathrm{H}), 6.72(\mathrm{~d}, J=6.0 \mathrm{~Hz}, 1 \mathrm{H}), 4.0-4.11(\mathrm{~m}, 1 \mathrm{H}), 2.10-2.14(\mathrm{~m}$, $2 \mathrm{H}), 1.85-1.90(\mathrm{~m}, 2 \mathrm{H}), 1.79-1.67(\mathrm{~m}, 1 \mathrm{H}), 1.48-1.54(\mathrm{~m}$, $2 \mathrm{H}), 1.47-1.30(\mathrm{~m}, 3 \mathrm{H}) .{ }^{13} \mathrm{C}$ NMR $(126 \mathrm{MHz}, \mathrm{MeOD}) \delta$ $162.93,155.06,154.25,151.12,144.26,141.28,136.28,136.14$, $127.94,127.20,120.72,114.97,107.81,49.92,31.93,25.34$, 24.63.
2-Chloro-N-methylpyrimidin-4-amine (3b). A mixture of 2,4-dichloropyrimidine 2 ( $10 \mathrm{mmol}, 1.0$ equiv) and methylamine $(2.0 \mathrm{M}$ in $\mathrm{MeOH})(20 \mathrm{mmol}, 2.0$ equiv) was stirred at rt in DCM $(20 \mathrm{~mL}, 0.50 \mathrm{M})$ in a nitrogen atmosphere. After stirring for $16 \mathrm{~h}$, the reaction mixture was concentrated to remove volatiles. Purification using automated flash chromatography (EtOAc/ hexanes $+2 \% \mathrm{MeOH}$ additive) was followed by evaporation, giving $\mathbf{3 b}$ as a white solid $(0.54 \mathrm{~g}, 38 \%)$. TLC $R_{f} 0.4(70 \%$ EtOAc/hexanes). LC-MS (ESI) $m / z: 144[\mathrm{M}+\mathrm{H}]^{+} .{ }^{1} \mathrm{H}$ NMR $(500 \mathrm{MHz}, \mathrm{MeOD}) \delta 7.81(\mathrm{~d}, J=6.0 \mathrm{~Hz}, 1 \mathrm{H}), 6.40(\mathrm{~d}, J=6.4 \mathrm{~Hz}$, $1 \mathrm{H}), 2.92(\mathrm{~s}, 3 \mathrm{H}) .{ }^{13} \mathrm{C}$ NMR (126 MHz, MeOD) $\delta$ 164.37, 160.27, 153.78, 104.47, 26.17.

$N^{4}$-Methyl- $N^{2}-(3-($ pyridin-3-yl)phenyl)pyrimidine-2,4-diamine (5a). To a mixture of $3 \mathbf{b}(0.10 \mathrm{mmol}, 1.0$ equiv) in $\mathrm{EtOH}$ $(0.50 \mathrm{~mL}, 0.20 \mathrm{M})$ under a nitrogen atmosphere at rt were added 3 -(pyridin-3-yl)aniline ( $0.11 \mathrm{mmol}, 1.1$ equiv) and a drop of $1 \mathrm{~N}$ $\mathrm{HCl}$. The reaction mixture was heated to reflux and stirred at that temperature for $3 \mathrm{~h}$; it was then cooled to rt. Purification using automated flash chromatography $(\mathrm{MeOH} / \mathrm{DCM})$ was followed by evaporation, giving $5 \mathrm{a}$ as a colorless oil $(0.006 \mathrm{~g}, 22 \%)$. TLC $R_{f}$ $0.4(10 \% \mathrm{MeOH} / \mathrm{DCM})$. LC-MS (ESI) $m / z: 278[\mathrm{M}+\mathrm{H}]^{+}{ }^{1} \mathrm{H}$ NMR (500 MHz, MeOD) $\delta 8.89-8.77(\mathrm{~m}, 1 \mathrm{H}), 8.52(\mathrm{~d}, J=5.0$ $\mathrm{Hz}, 1 \mathrm{H}), 8.24-8.05(\mathrm{~m}, 2 \mathrm{H}), 7.87-7.71(\mathrm{~m}, 1 \mathrm{H}), 7.67$ (ddd, $J=$ $8.1,1.8,0.9 \mathrm{~Hz}, 1 \mathrm{H}), 7.52(\mathrm{dd}, J=8.0,4.9 \mathrm{~Hz}, 1 \mathrm{H}), 7.41(\mathrm{t}, J=7.9$ $\mathrm{Hz}, 1 \mathrm{H}), 7.25$ (dt, $J=7.6,1.2 \mathrm{~Hz}, 1 \mathrm{H}), 5.98(\mathrm{~d}, J=6.0 \mathrm{~Hz}, 1 \mathrm{H})$, $2.95(\mathrm{~s}, 3 \mathrm{H}) .{ }^{13} \mathrm{C}$ NMR $(126 \mathrm{MHz}, \mathrm{MeOD}) \delta 163.84,159.69$, 153.38 , 147.22, 146.94, 141.50, 137.51, 135.13, 129.06, 124.02, 119.79, 118.90, 117.48, 97.46, 26.56.

2-Chloro-N-ethylpyrimidin-4-amine (3c). A mixture of 2,4dichloropyrimidine 2 (10 mmol, 1.0 equiv) and ethylamine (2.0 $\mathrm{M}$ in THF) (20 mmol, 2.0 equiv) was stirred at $\mathrm{rt}$ in $\mathrm{MeOH}$ (10 $\mathrm{mL}, 1.0 \mathrm{M}$ ) in a nitrogen atmosphere. After $16 \mathrm{~h}$, the reaction mixture was concentrated to remove volatiles. Purification using automated flash chromatography (EtOAc/hexanes $+2 \% \mathrm{MeOH}$ additive) was followed by evaporation, giving $3 \mathrm{c}$ as a white solid (0.43 g, 27\%). TLC $R_{f} 0.5$ (70\% EtOAc/hexanes). LC-MS (ESI) $m / z: 160[\mathrm{M}+\mathrm{H}]^{+} .{ }^{1} \mathrm{H}$ NMR $\left(500 \mathrm{MHz}, \mathrm{CDCl}_{3}\right) \delta 8.04$ (br s, $1 \mathrm{H}), 6.26(\mathrm{~d}, J=5.9 \mathrm{~Hz}, 1 \mathrm{H}), 5.41(\mathrm{br} \mathrm{s}, 1 \mathrm{H}), 3.38(\mathrm{br} \mathrm{s}$, $2 \mathrm{H}), 1.28(\mathrm{t}, J=7.3 \mathrm{~Hz}, 3 \mathrm{H}) .{ }^{13} \mathrm{C} \mathrm{NMR}\left(126 \mathrm{MHz}, \mathrm{CDCl}_{3}\right) \delta$ $163.55,160.58,157.26,99.99,36.48,14.36$.

$N^{4}$-Ethyl- $N^{2}-(3-($ pyridin-3-yl)phenyl)pyrimidine-2,4-diamine (5b). To a mixture of $3 \mathrm{c}(0.10 \mathrm{mmol}, 1.0$ equiv) in EtOH $(0.50 \mathrm{~mL}, 0.20 \mathrm{M})$ under a nitrogen atmosphere at rt were added 3-(pyridin-3-yl)aniline ( $0.11 \mathrm{mmol}, 1.1$ equiv) and a drop of $1 \mathrm{~N}$ $\mathrm{HCl}$. The reaction mixture was heated to reflux and stirred at that temperature for $3 \mathrm{~h}$; it was then cooled to rt. Purification using automated flash chromatography $(\mathrm{MeOH} / \mathrm{DCM})$ was followed by evaporation, giving $\mathbf{5 b}$ as a colorless oil $(0.003 \mathrm{~g}, 10 \%)$. TLC $R_{f} 0.1$ (5\% MeOH/DCM). LC-MS (ESI) $m / z: 292[\mathrm{M}+\mathrm{H}]^{+}$. ${ }^{1} \mathrm{H}$ NMR (500 MHz, MeOD) $\delta 8.84(\mathrm{~s}, 1 \mathrm{H}), 8.55(\mathrm{~s}, 1 \mathrm{H}), 8.18$ (s, $1 \mathrm{H}), 8.13(\mathrm{dt}, J=8.1,1.8 \mathrm{~Hz}, 1 \mathrm{H}), 7.78(\mathrm{~s}, 1 \mathrm{H}), 7.68-7.59$ $(\mathrm{m}, 1 \mathrm{H}), 7.55(\mathrm{dd}, J=8.0,4.8 \mathrm{~Hz}, 1 \mathrm{H}), 7.42(\mathrm{t}, J=7.9 \mathrm{~Hz}, 1 \mathrm{H})$, $7.26(\mathrm{dt}, J=7.7,1.2 \mathrm{~Hz}, 1 \mathrm{H}), 5.97(\mathrm{~d}, J=5.4 \mathrm{~Hz}, 1 \mathrm{H}), 3.56-3.38$ $(\mathrm{m}, 2 \mathrm{H}), 1.23(\mathrm{t}, J=7.2 \mathrm{~Hz}, 3 \mathrm{H}) .{ }^{13} \mathrm{C}$ NMR $(126 \mathrm{MHz}, \mathrm{MeOD})$ $\delta 163.10,147.24,146.98,141.55,137.81,137.57,135.18,129.04$, $124.04,119.79,118.83,117.43,35.14,13.49$.

2-Chloro-N-propylpyrimidin-4-amine (3d). A mixture of 2,4dichloropyrimidine 2 (10 mmol, 1.0 equiv) and cyclopentylamine (20 mmol, 2.0 equiv) was stirred at rt in DCM $(20 \mathrm{~mL}$, $0.50 \mathrm{M})$ in a nitrogen atmosphere. After stirring for $16 \mathrm{~h}$, the reaction mixture was concentrated. Purification using automated flash chromatography (EtOAc/hexanes) was followed by evaporation, giving $3 \mathbf{d}$ as a colorless oil $(0.84 \mathrm{~g}, 49 \%)$. TLC $R_{f}$ 
0.3 (40\% EtOAc/hexanes). LC-MS (ESI) $m / z: 174[\mathrm{M}+\mathrm{H}]^{+}$. ${ }^{1} \mathrm{H}$ NMR (500 MHz, CDCl ${ }_{3}$ ) $\delta 8.04$ (br s, $\left.1 \mathrm{H}\right), 6.27$ (d, $J=6.0$ $\mathrm{Hz}, 1 \mathrm{H}), 5.54($ br s, $1 \mathrm{H}), 3.28(\mathrm{br} \mathrm{s}, 2 \mathrm{H}), 1.66(\mathrm{q}, J=7.3 \mathrm{~Hz}, 2 \mathrm{H})$, $1.00(\mathrm{t}, J=7.4 \mathrm{~Hz}, 3 \mathrm{H}) .{ }^{13} \mathrm{C} \mathrm{NMR}\left(126 \mathrm{MHz}, \mathrm{CDCl}_{3}\right) \delta 163.74$, $160.55,157.72,99.81,43.44,22.35,11.32$.

$N^{4}$-Propyl- $N^{2}$-(3-(pyridin-3-yl)phenyl)pyrimidine-2,4-diamine (5c). To a mixture of $3 \mathrm{~d}(0.10 \mathrm{mmol}, 1.0$ equiv) in $\mathrm{EtOH}$ $(0.50 \mathrm{~mL}, 0.20 \mathrm{M})$ under a nitrogen atmosphere at $\mathrm{rt}$ were added 3-(pyridin-3-yl)aniline ( $0.11 \mathrm{mmol}, 1.1$ equiv) and a drop of $1 \mathrm{~N}$ $\mathrm{HCl}$. The reaction mixture was heated to reflux and stirred at that temperature for $3 \mathrm{~h}$; it was then cooled to rt. Purification using automated flash chromatography $(\mathrm{MeOH} / \mathrm{DCM})$ was followed by evaporation, giving $5 \mathrm{c}$ as a colorless oil $(0.005 \mathrm{~g}, 16 \%)$. TLC $R_{f}$ $0.4(10 \% \mathrm{MeOH} / \mathrm{DCM})$. LC-MS (ESI) $m / z: 306[\mathrm{M}+\mathrm{H}]^{+} .{ }^{1} \mathrm{H}$ NMR $(500 \mathrm{MHz}, \mathrm{MeOD}) \delta 8.82(\mathrm{~s}, 1 \mathrm{H}), 8.58-8.47(\mathrm{~m}, 1 \mathrm{H})$, 8.21-8.07 (m, 2H), $7.76(\mathrm{~s}, 1 \mathrm{H}), 7.69-7.61(\mathrm{~m}, 1 \mathrm{H}), 7.53(\mathrm{dd}, J$ $=7.9,4.8 \mathrm{~Hz}, 1 \mathrm{H}), 7.41(\mathrm{t}, J=7.9 \mathrm{~Hz}, 1 \mathrm{H}), 7.25(\mathrm{dt}, J=7.7,1.2$ $\mathrm{Hz}, 1 \mathrm{H}), 5.97$ (d, $J=5.8 \mathrm{~Hz}, 1 \mathrm{H}), 3.37$ (s, 2H), 1.62 (q, $J=7.3$ $\mathrm{Hz}, 2 \mathrm{H}), 0.92(\mathrm{t}, J=7.3 \mathrm{~Hz}, 3 \mathrm{H}) .{ }^{13} \mathrm{C} \mathrm{NMR}(126 \mathrm{MHz}, \mathrm{MeOD})$ $\delta 163.26,159.66,153.40,147.22,147.00,141.51,137.76,137.56$, $135.19,129.02,124.02,119.84,118.89,117.53,97.60,42.24$, $22.19,10.38$.

N-Butyl-2-chloropyrimidin-4-amine (3e). To a suspension of 2,4-dichloropyrimidine 2 ( $10 \mathrm{mmol}, 1.0$ equiv) in DCM $(20 \mathrm{~mL}$, $0.50 \mathrm{M})$ was added butylamine $(20 \mathrm{mmol}, 2.0$ equiv) under a nitrogen atmosphere at rt. After stirring for $16 \mathrm{~h}$, the reaction mixture was concentrated. Purification using automated flash chromatography (EtOAc/hexanes) was followed by evaporation, giving $3 \mathrm{e}$ as a white solid (0.96 g, 52\%). TLC $R_{f} 0.25(30 \%$ EtOAc/hexanes). LC-MS (ESI) $m / z: 188[\mathrm{M}+\mathrm{H}]^{+} .{ }^{1} \mathrm{H}$ NMR $\left(400 \mathrm{MHz}, \mathrm{CDCl}_{3}\right) \delta 8.04$ (br s, $\left.1 \mathrm{H}\right), 6.25(\mathrm{~d}, J=5.9 \mathrm{~Hz}, 1 \mathrm{H})$, 5.46 (br s, $1 \mathrm{H}), 3.31$ (br s, $2 \mathrm{H}), 1.71-1.51(\mathrm{~m}, 2 \mathrm{H}), 1.51-1.33$ $(\mathrm{m}, 2 \mathrm{H}), 0.97(\mathrm{t}, J=7.3 \mathrm{~Hz}, 3 \mathrm{H}) .{ }^{13} \mathrm{C} \mathrm{NMR}\left(101 \mathrm{MHz}, \mathrm{CDCl}_{3}\right) \delta$ 163.70, 160.62, 156.38, 41.42, 31.11, 19.97, 13.71.

$N^{4}$-Butyl- $N^{2}-(3-($ pyridin-3-yl)phenyl)pyrimidine-2,4-diamine (5d). To a mixture of $3 \mathrm{e}(0.10 \mathrm{mmol}, 1.0$ equiv $)$ in $\mathrm{EtOH}$ $(0.50 \mathrm{~mL}, 0.20 \mathrm{M})$ under a nitrogen atmosphere at $\mathrm{rt}$ were added 3-(pyridin-3-yl)aniline ( $0.11 \mathrm{mmol}, 1.1$ equiv) and a drop of $1 \mathrm{~N}$ $\mathrm{HCl}$. The reaction mixture was heated to reflux and stirred at that temperature for $3 \mathrm{~h}$; it was then cooled to rt. Purification using automated flash chromatography $(\mathrm{MeOH} / \mathrm{DCM})$ was followed by evaporation, giving $\mathbf{5 d}$ as a colorless oil $(0.006 \mathrm{~g}, 19 \%)$. TLC $R_{f} 0.4(10 \% \mathrm{MeOH} / \mathrm{DCM})$. LC-MS (ESI) $\mathrm{m} / z: 320[\mathrm{M}+\mathrm{H}]^{+}$. ${ }^{1} \mathrm{H} \mathrm{NMR}(400 \mathrm{MHz}, \mathrm{MeOD}) \delta 8.70(\mathrm{~s}, 1 \mathrm{H}), 8.48-8.33(\mathrm{~m}, 1 \mathrm{H})$, 8.07-7.95 (m, 2H), $7.63(\mathrm{~s}, 1 \mathrm{H}), 7.53(\mathrm{dd}, J=8.2,2.1 \mathrm{~Hz}, 1 \mathrm{H})$, $7.39-7.43(\mathrm{~m}, 1 \mathrm{H}), 7.29(\mathrm{t}, J=7.9 \mathrm{~Hz}, 1 \mathrm{H}), 7.13(\mathrm{~d}, J=7.7 \mathrm{~Hz}$, $1 \mathrm{H}), 5.85(\mathrm{~d}, J=6.0 \mathrm{~Hz}, 1 \mathrm{H}), 3.28(\mathrm{~s}, 2 \mathrm{H}), 1.43-1.50(\mathrm{~m}, 2 \mathrm{H})$, $1.21-1.26(\mathrm{~m}, 2 \mathrm{H}), 0.79(\mathrm{t}, J=7.4 \mathrm{~Hz}, 3 \mathrm{H}) .{ }^{13} \mathrm{C} \mathrm{NMR}(101$ $\mathrm{MHz}, \mathrm{MeOD}) \delta 164.65,161.00,148.65,148.45,142.88,139.17$, $136.60,130.43,125.42,121.32,120.36,119.03,117.61,41.58$, $32.56,21.21,14.19$.

2-Chloro-N-isopropylpyrimidin-4-amine (3f). To a suspension of 2,4-dichloropyrimidine 2 ( $10 \mathrm{mmol}, 1.0$ equiv) in DCM $(20 \mathrm{~mL}, 0.50 \mathrm{M})$ under a nitrogen atmosphere was added isopropylamine ( $20 \mathrm{mmol}, 2.0$ equiv) at rt. After stirring for $16 \mathrm{~h}$, the reaction mixture was concentrated. Purification using automated flash chromatography (EtOAc/hexanes) was followed by evaporation, giving $3 \mathrm{f}$ as a colorless oil (1.0 g, 60\%). TLC $R_{f} 0.10$ (30\% EtOAc/hexanes). LC-MS (ESI) $m / z: 174$ $[\mathrm{M}+\mathrm{H}]^{+} .{ }^{1} \mathrm{H}$ NMR $\left(400 \mathrm{MHz}, \mathrm{CDCl}_{3}\right) \delta 8.02(\mathrm{~s}, 1 \mathrm{H}), 6.23(\mathrm{~d}, J$ $=5.9 \mathrm{~Hz}, 1 \mathrm{H}), 5.10(\mathrm{br} \mathrm{s}, 1 \mathrm{H}), 3.94(\mathrm{br} \mathrm{s}, 1 \mathrm{H}), 1.27(\mathrm{~d}, J=6.5 \mathrm{~Hz}$, $6 \mathrm{H}) .{ }^{13} \mathrm{C} \mathrm{NMR}\left(126 \mathrm{MHz}, \mathrm{CDCl}_{3}\right) \delta 162.81,160.84,157.19$, $100.94,43.25,22.52$.
$N^{4}$-Isopropyl- $N^{2}$-(3-(pyridin-3-yl)phenyl)pyrimidine-2,4-diamine (5e). To a mixture of $3 \mathrm{f}(0.105 \mathrm{mmol}, 1.00$ equiv) in $\mathrm{EtOH}(0.500 \mathrm{~mL}, 0.210 \mathrm{M})$ under a nitrogen atmosphere at $\mathrm{rt}$ were added 3-(pyridin-3-yl)aniline $(0.115 \mathrm{mmol}, 1.10$ equiv) and a drop of $1 \mathrm{~N} \mathrm{HCl}$. The reaction mixture was heated to reflux and stirred at that temperature for $3 \mathrm{~h}$; it was then cooled to $\mathrm{rt}$. Purification using automated flash chromatography $(\mathrm{MeOH} /$ DCM) was followed by evaporation, giving $5 \mathbf{e}$ as a colorless oil (0.005 g, 16\%). TLC $R_{f} 0.5$ (10\% MeOH/DCM). HRMS (ESI) $\mathrm{m} / z$ : calcd for $[\mathrm{M}+\mathrm{H}]^{+} 306.1718$, found $306.1720 .{ }^{1} \mathrm{H}$ NMR $\left(500 \mathrm{MHz}, \mathrm{CDCl}_{3}\right) \delta 8.87(\mathrm{~s}, 1 \mathrm{H}), 8.59(\mathrm{~s}, 1 \mathrm{H}), 8.05-7.82(\mathrm{~m}$, $3 \mathrm{H}), 7.55(\mathrm{~d}, J=5.0 \mathrm{~Hz}, 1 \mathrm{H}), 7.35-7.41(\mathrm{~m}, 3 \mathrm{H}), 7.20(\mathrm{~d}, J=$ $10.0 \mathrm{~Hz}, 1 \mathrm{H}), 5.85(\mathrm{~d}, J=5.7 \mathrm{~Hz}, 1 \mathrm{H}), 4.72(\mathrm{~s}, 1 \mathrm{H}), 4.06(\mathrm{~s}, 1 \mathrm{H})$, $1.25(\mathrm{~d}, J=6.4 \mathrm{~Hz}, 6 \mathrm{H}) .{ }^{13} \mathrm{C} \mathrm{NMR}\left(126 \mathrm{MHz}, \mathrm{CDCl}_{3}\right) \delta 162.16$, $159.77,155.62,148.38,148.36,141.01,138.40,136.95,134.44$, 129.41, 123.46, 120.54, 118.80, 117.78, 96.46, 42.81, 22.80.

$\mathrm{N}$-(tert-Butyl)-2-chloropyrimidin-4-amine (3g). A mixture of 2,4-dichloropyrimidine 2 (10 mmol, 1.0 equiv) and tertbutylamine ( $20 \mathrm{mmol}, 2.0$ equiv) was stirred at rt in DCM (20 $\mathrm{mL}, 0.50 \mathrm{M}$ ) under a nitrogen atmosphere for $16 \mathrm{~h}$. The reaction mixture was then concentrated to remove volatiles. Purification using automated flash chromatography (EtOAc/hexanes) was followed by evaporation, giving $\mathbf{3 g}$ as a white oil $(0.017 \mathrm{~g}, 1 \%)$. TLC $R_{f} 0.3$ (40\% EtOAc/hexanes). LC-MS (ESI) $m / z: 186[\mathrm{M}$ $+\mathrm{H}]^{+} .{ }^{1} \mathrm{H}$ NMR $\left(500 \mathrm{MHz}, \mathrm{CDCl}_{3}\right) \delta 7.97(\mathrm{~d}, J=6.0 \mathrm{~Hz}, 1 \mathrm{H})$, 6.25 (br s, $1 \mathrm{H}), 5.07$ (br s, $1 \mathrm{H}), 1.44$ (s, 9H). ${ }^{13} \mathrm{C}$ NMR $(126$ $\left.\mathrm{MHz}, \mathrm{CDCl}_{3}\right) \delta 162.88,160.49,156.53,103.33,51.87,28.90$.

$N^{4}$-(tert-Butyl)- $N^{2}$-(3-(pyridin-3-yl)phenyl)pyrimidine-2,4diamine (5f). To a mixture of $3 \mathrm{~g}(0.081 \mathrm{mmol}, 1.0$ equiv $)$ in $\mathrm{EtOH}(0.50 \mathrm{~mL}, 0.16 \mathrm{M})$ under a nitrogen atmosphere at rt were added 3-(pyridin-3-yl)aniline (0.089 mmol, 1.1 equiv) and a drop of $1 \mathrm{~N} \mathrm{HCl}$. The reaction mixture was heated to reflux and stirred at that temperature for $3 \mathrm{~h}$; it was then cooled to rt. Purification using automated flash chromatography $(\mathrm{MeOH} /$ DCM) was followed by evaporation, giving $\mathbf{5 f}$ as an orange oil (0.004 g, 16\%). TLC $R_{f} 0.3$ (5\% MeOH/DCM). LC-MS (ESI) $m / z: 319[\mathrm{M}+\mathrm{H}]^{+} .{ }^{1} \mathrm{H}$ NMR $\left(500 \mathrm{MHz}, \mathrm{CDCl}_{3}\right) \delta 8.91$ (br s, $1 \mathrm{H}), 8.63$ (br s, $1 \mathrm{H}), 8.08-7.73(\mathrm{~m}, 3 \mathrm{H}), 7.58(\mathrm{~d}, J=8.2 \mathrm{~Hz}$, $1 \mathrm{H}), 7.40-7.44(\mathrm{~m}, 3 \mathrm{H}), 7.24(\mathrm{~d}, J=5.0 \mathrm{~Hz}, 1 \mathrm{H}), 5.89(\mathrm{~d}, J=5.4$ $\mathrm{Hz}, 1 \mathrm{H}), 4.85$ (s, 1H), 1.46 (s, 9H). ${ }^{13} \mathrm{C}$ NMR (126 MHz, $\left.\mathrm{CDCl}_{3}\right) \delta 162.37,158.85,153.78,148.44,140.43,138.58,134.51$, 129.44, 123.54, 121.10, 119.34, 118.31, 98.58, 51.59, 29.14.

2-Chloro-N-(1-methoxypropan-2-yl)pyrimidin-4-amine (3h). A mixture of 2,4-dichloropyrimidine 2 (5.0 mmol, 1.0 equiv), 1-methoxypropan-2-amine $\mathrm{HCl}$ ( $7.5 \mathrm{mmol}, 1.5$ equiv), and triethylamine (10.5 mmol, 2.1 equiv) was stirred at $\mathrm{rt}$ in $\operatorname{DCM}(10 \mathrm{~mL}, 0.50 \mathrm{M})$ in a nitrogen atmosphere. After $16 \mathrm{~h}$, the reaction mixture was concentrated to remove volatiles. To the crude residue was added water $(10 \mathrm{~mL})$, and the aqueous portion was extracted into EtOAc $(3 \times 10 \mathrm{~mL})$. The combined organics were dried over magnesium sulfate, filtered, and concentrated. Purification using automated flash chromatography (EtOAc/ hexanes) was followed by evaporation, giving $3 \mathbf{h}$ as a colorless oil (0.29 g, 29\%). TLC $R_{f} 0.3$ (60\% EtOAc/hexanes). LC-MS (ESI) $m / z: 204[\mathrm{M}+\mathrm{H}]^{+} .{ }^{1} \mathrm{H}$ NMR $\left(500 \mathrm{MHz}, \mathrm{CDCl}_{3}\right) \delta 8.00$ $(\mathrm{d}, J=6.2 \mathrm{~Hz}, 1 \mathrm{H}), 6.26(\mathrm{~d}, J=6.0 \mathrm{~Hz}, 1 \mathrm{H}), 5.44(\mathrm{br} \mathrm{s}, 1 \mathrm{H}), 4.21$ (br s, $1 \mathrm{H}), 3.56-3.31(\mathrm{~m}, 5 \mathrm{H}), 1.28(\mathrm{~d}, J=6.7 \mathrm{~Hz}, 3 \mathrm{H}) .{ }^{13} \mathrm{C}$ NMR $\left(126 \mathrm{MHz}, \mathrm{CDCl}_{3}\right) \delta 162.99,160.71,156.53,104.44$, 75.32, 59.18, 46.45, 17.43 .

$N^{4}$-(1-Methoxypropan-2-yl)- $N^{2}-(3-($ pyridin-3-yl)phenyl)pyrimidine-2,4-diamine $(5 \mathrm{~g})$. To a mixture of $3 \mathrm{~h}(0.10 \mathrm{mmol}$, 1.0 equiv) in $\mathrm{EtOH}(0.50 \mathrm{~mL}, 0.20 \mathrm{M})$ under a nitrogen atmosphere at rt were added 3-(pyridin-3-yl)aniline $(0.11 \mathrm{mmol}$, 
1.1 equiv) and a drop of $1 \mathrm{~N} \mathrm{HCl}$. The reaction mixture was heated to reflux and stirred at that temperature for $3 \mathrm{~h}$; it was then cooled to rt. Purification using automated flash chromatography ( $\mathrm{MeOH} / \mathrm{DCM})$ was followed by evaporation, giving $\mathbf{5 g}$ as a colorless oil ( $0.010 \mathrm{~g}, 30 \%)$. TLC $R_{f} 0.2$ (5\% MeOH/DCM). LC-MS (ESI) $m / z: 336[\mathrm{M}+\mathrm{H}]^{+}$. ${ }^{1} \mathrm{H}$ NMR $(500 \mathrm{MHz}$, $\mathrm{MeOD}) \delta 8.83(\mathrm{~s}, 1 \mathrm{H}), 8.54(\mathrm{~d}, J=4.9 \mathrm{~Hz}, 1 \mathrm{H}), 8.11-8.14(\mathrm{~m}$, $2 \mathrm{H}), 7.76(\mathrm{~d}, J=5.7 \mathrm{~Hz}, 1 \mathrm{H}), 7.63(\mathrm{~d}, J=5.0 \mathrm{~Hz}, 1 \mathrm{H}), 7.54(\mathrm{ddd}$, $J=7.9,4.9,0.9 \mathrm{~Hz}, 1 \mathrm{H}), 7.41(\mathrm{t}, J=7.9 \mathrm{~Hz}, 1 \mathrm{H}), 7.26(\mathrm{~d}, J=5.0$ $\mathrm{Hz}, 1 \mathrm{H}), 5.99$ (d, $J=6.0 \mathrm{~Hz}, 1 \mathrm{H}), 4.38(\mathrm{~s}, 1 \mathrm{H}), 3.42$ (ddd, $J=$ 49.5, 9.4, 5.6 Hz, 2H), $3.33(\mathrm{~s}, 3 \mathrm{H}), 1.23(\mathrm{~d}, J=6.7 \mathrm{~Hz}, 3 \mathrm{H}) .{ }^{13} \mathrm{C}$ NMR (126 MHz, MeOD) $\delta 162.75,159.70,153.82,147.24$, $147.00,141.52,137.59,135.24,129.04,124.01,119.84,118.89$, $117.51,97.54,75.41,57.78,45.46,16.36$.

Methyl (2-chloropyrimidin-4-yl)-L-alaninate (3i). A mixture of 2,4-dichloropyrimidine 2 ( $10 \mathrm{mmol}, 1.0$ equiv), H-Ala-OMe$\mathrm{HCl}$ (10 mmol, 1.0 equiv), and triethylamine ( $12 \mathrm{mmol}, 1.2$ equiv) was stirred at rt in DCM $(20 \mathrm{~mL}, 0.50 \mathrm{M})$ in a nitrogen atmosphere. After stirring for $16 \mathrm{~h}$, the reaction mixture was concentrated to remove volatiles. To the crude residue was added water $(10 \mathrm{~mL})$, and the crude was extracted into EtOAc (3 $\times 10 \mathrm{~mL}$ ). The combined organic phases were dried over magnesium sulfate, filtered, and concentrated. Purification using automated flash chromatography (EtOAc/hexanes) was followed by evaporation, giving $3 \mathbf{i}$ as a colorless oil $(0.13 \mathrm{~g}, 6 \%)$. TLC $R_{f} 0.2$ (40\% EtOAc/hexanes). LC-MS (ESI) $m / z: 218[\mathrm{M}$ $+\mathrm{H}]^{+} \cdot[\alpha]_{\mathrm{D}}{ }^{20}=-20\left(c 0.00066, \mathrm{CHCl}_{3}\right) \cdot{ }^{1} \mathrm{H} \mathrm{NMR}(500 \mathrm{MHz}$, $\left.\mathrm{CDCl}_{3}\right) \delta 8.15-7.97(\mathrm{~m}, 1 \mathrm{H}), 6.33(\mathrm{~d}, J=5.4 \mathrm{~Hz}, 1 \mathrm{H}), 5.69(\mathrm{br} \mathrm{s}$, $1 \mathrm{H}), 4.73$ (br s, $1 \mathrm{H}), 3.82(\mathrm{~s}, 3 \mathrm{H}), 1.54(\mathrm{~d}, J=5.0 \mathrm{~Hz}, 3 \mathrm{H}) .{ }^{13} \mathrm{C}$ NMR $\left(126 \mathrm{MHz}, \mathrm{CDCl}_{3}\right) \delta 173.35,162.39,160.59,160.54$, $156.33,104.28,52.74,49.33,18.31$.

Methyl (2-((3-(pyridin-3-yl)phenyl)amino)pyrimidin-4-yl)-Lalaninate (5h). To a mixture of $3 \mathbf{i}(0.30 \mathrm{mmol}, 1.0$ equiv $)$ in $\mathrm{EtOH}(1.5 \mathrm{~mL}, 0.20 \mathrm{M})$ under a nitrogen atmosphere at rt were added 3-(pyridin-3-yl)aniline ( $0.33 \mathrm{mmol}, 1.1$ equiv) and a drop of $1 \mathrm{~N} \mathrm{HCl}$. The reaction mixture was heated to reflux and stirred at that temperature for $3 \mathrm{~h}$; it was then cooled to rt. Purification using automated flash chromatography $(\mathrm{MeOH} / \mathrm{DCM})$ was followed by evaporation, giving $\mathbf{5 h}$ as an orange foam $(0.024 \mathrm{~g}$, 23\%). TLC $R_{f} 0.1$ (5\% MeOH/DCM). LC-MS (ESI) $m / z: 350$ $[\mathrm{M}+\mathrm{H}]^{+} .[\alpha]_{\mathrm{D}}{ }^{20}=-36\left(c 0.000044, \mathrm{CHCl}_{3}\right) .{ }^{1} \mathrm{H} \operatorname{NMR}(500$ $\mathrm{MHz}, \mathrm{MeOD}) \delta 8.85(\mathrm{~s}, 1 \mathrm{H}), 8.54(\mathrm{~d}, J=4.9 \mathrm{~Hz}, 1 \mathrm{H}), 8.14(\mathrm{dt}, J$ $=8.0,1.9 \mathrm{~Hz}, 1 \mathrm{H}), 7.97(\mathrm{t}, J=2.0 \mathrm{~Hz}, 1 \mathrm{H}), 7.82(\mathrm{~d}, J=5.9 \mathrm{~Hz}$, $1 \mathrm{H}), 7.66(\mathrm{ddd}, J=8.4,2.2,1.0 \mathrm{~Hz}, 1 \mathrm{H}), 7.55(\mathrm{dd}, J=7.9,4.9 \mathrm{~Hz}$, $1 \mathrm{H}), 7.42(\mathrm{t}, J=7.9 \mathrm{~Hz}, 1 \mathrm{H}), 7.28(\mathrm{ddd}, J=7.7,1.8,1.0 \mathrm{~Hz}, 1 \mathrm{H})$, $6.09(\mathrm{~d}, J=5.9 \mathrm{~Hz}, 1 \mathrm{H}), 4.68(\mathrm{~d}, J=7.3 \mathrm{~Hz}, 1 \mathrm{H}), 3.57(\mathrm{~s}, 3 \mathrm{H})$, $1.48(\mathrm{~d}, J=7.3 \mathrm{~Hz}, 3 \mathrm{H}) .{ }^{13} \mathrm{C}$ NMR $(126 \mathrm{MHz}, \mathrm{MeOD}) \delta 174.42$, $162.57,159.21,153.59,147.25,147.05,141.08,137.64,135.25$, $129.08,124.01,120.21,119.23,117.75,97.64,51.11,49.18$, 48.10, 16.28 .

(2-((3-(Pyridin-3-yl)phenyl)amino)pyrimidin-4-yl)-L-alanine (5i). To $5 \mathrm{~h}$ ( $0.043 \mathrm{mmol}, 1.0$ equiv) in $0.72 \mathrm{~mL}(0.060 \mathrm{M})$ of THF/water (5:1) at rt under a nitrogen atmosphere was added a $1 \mathrm{~N} \mathrm{NaOH}$ solution $(0.13 \mathrm{mmol}, 3.0$ equiv). The reaction mixture was stirred at $80^{\circ} \mathrm{C}$ for $3 \mathrm{~h}$; it was then cooled to rt and concentrated to remove volatiles. The residue was diluted with water $(1 \mathrm{~mL})$ and then adjusted to $\mathrm{pH} 4$ with $1 \mathrm{~N} \mathrm{HCl}$. The precipitated solid was filtered and washed with water, providing 5i as a white solid $(0.007 \mathrm{~g}, 49 \%)$. LC-MS (ESI) $m / z: 336[\mathrm{M}+$ $\mathrm{H}]^{+} .[\alpha]_{\mathrm{D}}{ }^{20}=-55\left(c 0.000024, \mathrm{CHCl}_{3}\right) .{ }^{1} \mathrm{H}$ NMR $(500 \mathrm{MHz}$, $\mathrm{MeOD}) \delta 8.81(\mathrm{~s}, 1 \mathrm{H}), 8.54(\mathrm{~s}, 1 \mathrm{H}), 8.16-8.06(\mathrm{~m}, 1 \mathrm{H}), 7.72$ $(\mathrm{dd}, J=5.6,2.8 \mathrm{~Hz}, 2 \mathrm{H}), 7.64(\mathrm{~d}, J=6.8 \mathrm{~Hz}, 1 \mathrm{H}), 7.54(\mathrm{dd}, J=$ 8.0, $4.9 \mathrm{~Hz}, 1 \mathrm{H}), 7.47(\mathrm{t}, J=8.1 \mathrm{~Hz}, 1 \mathrm{H}), 7.37(\mathrm{dt}, J=7.8,1.4 \mathrm{~Hz}$,
$1 \mathrm{H}), 6.18(\mathrm{~d}, J=6.7 \mathrm{~Hz}, 1 \mathrm{H}), 4.51(\mathrm{~s}, 1 \mathrm{H}), 1.49(\mathrm{~d}, J=7.2 \mathrm{~Hz}$, $3 \mathrm{H}) .{ }^{13} \mathrm{C}$ NMR (126 MHz, MeOD) $\delta 176.99,162.40,155.33$, $147.46,146.98,139.06,137.96,136.98,135.26,129.59,124.10$, 122.01, 120.78, 119.07, 98.50, 51.06, 16.82 .

(S)-N-(sec-Butyl)-2-chloropyrimidin-4-amine (3j). To a suspension of 2,4-dichloropyrimidine 2 ( $2.0 \mathrm{mmol}, 1.0$ equiv) in DCM $(5.0 \mathrm{~mL}, 0.40 \mathrm{M})$ under a nitrogen atmosphere was added $(S)-(+)$-2-aminobutane (4.0 mmol, 2.0 equiv) at rt. After stirring for $6 \mathrm{~h}$, the reaction mixture was concentrated. Purification using automated flash chromatography (EtOAc/ hexanes) was followed by evaporation, giving $3 \mathbf{j}$ as a colorless oil (0.14 g, 37\%). TLC $R_{f} 0.25$ (30\% EtOAc/hexanes). LC-MS (ESI) $m / z: 188[\mathrm{M}+\mathrm{H}]^{+} .[\alpha]_{\mathrm{D}}{ }^{20}=29\left(c 0.0011, \mathrm{CHCl}_{3}\right) \cdot{ }^{1} \mathrm{H}$ $\operatorname{NMR}(500 \mathrm{MHz}, \mathrm{MeOD}) \delta 7.79(\mathrm{~d}, J=5.0 \mathrm{~Hz}, 1 \mathrm{H}), 6.36(\mathrm{~d}, J=$ $5.0 \mathrm{~Hz}, 1 \mathrm{H}), 4.04-4.09(\mathrm{~m}, 1 \mathrm{H}), 1.64-1.49(\mathrm{~m}, 2 \mathrm{H}), 1.19(\mathrm{~d}, J=$ $6.6 \mathrm{~Hz}, 3 \mathrm{H}), 0.95(\mathrm{t}, J=7.4 \mathrm{~Hz}, 3 \mathrm{H}) .{ }^{13} \mathrm{C}$ NMR $(126 \mathrm{MHz}$, MeOD) $\delta 163.46,160.18,153.91,104.38,28.82,18.75,9.38$.

(S)- $N^{4}$-(sec-Butyl)- $N^{2}-(3-($ pyridin-3-yl)phenyl)pyrimidine2,4-diamine (5j). To a mixture of $3 \mathbf{j}(0.10 \mathrm{mmol}, 1.0$ equiv) in $\mathrm{EtOH}(0.50 \mathrm{~mL}, 0.20 \mathrm{M})$ under a nitrogen atmosphere at rt were added 3-(pyridin-3-yl)aniline (0.11 mmol, 1.1 equiv) and a drop of $1 \mathrm{~N} \mathrm{HCl}$. The reaction mixture was heated to reflux and stirred at that temperature for $4 \mathrm{~h}$; it was then cooled to rt. Purification using automated flash chromatography $(\mathrm{MeOH} / \mathrm{DCM})$ was followed by evaporation, giving $\mathbf{5} \mathbf{j}$ as a yellow oil $(0.007 \mathrm{~g}, 22 \%)$. TLC $R_{f} 0.5$ (10\% MeOH/DCM). LC-MS (ESI) $m / z: 320[\mathrm{M}+$ $\mathrm{H}]^{+} .[\alpha]_{\mathrm{D}}{ }^{20}=11\left(c 0.000073, \mathrm{CHCl}_{3}\right) .{ }^{1} \mathrm{H}$ NMR $(500 \mathrm{MHz}$, $\left.\mathrm{CDCl}_{3}\right) \delta 8.86(\mathrm{~s}, 1 \mathrm{H}), 8.58(\mathrm{~d}, J=4.5 \mathrm{~Hz}, 1 \mathrm{H}), 8.01-7.80(\mathrm{~m}$, $3 \mathrm{H}), 7.57(\mathrm{~d}, J=5.0 \mathrm{~Hz}, 1 \mathrm{H}), 7.49-7.29(\mathrm{~m}, 3 \mathrm{H}), 7.19(\mathrm{~d}, J=5.8$ $\mathrm{Hz}, 1 \mathrm{H}), 5.85$ (d, J = 5.8 Hz, 1H), 4.77 (br s, $1 \mathrm{H}), 3.85$ (br s, $1 \mathrm{H})$, $1.53-1.59(\mathrm{~m}, 2 \mathrm{H}), 1.20(\mathrm{~d}, J=6.4 \mathrm{~Hz}, 3 \mathrm{H}), 0.92(\mathrm{t}, J=7.4 \mathrm{~Hz}$, $3 \mathrm{H}) .{ }^{13} \mathrm{C}$ NMR $\left(126 \mathrm{MHz}, \mathrm{CDCl}_{3}\right) \delta 162.43,159.48,155.08$, $148.41,140.82$, 138.47, 136.91, 134.45, 129.43, 123.46, 120.68, $118.81,117.79,96.43,48.24,29.66,20.27,10.36$.

(R)-N-(sec-Butyl)-2-chloropyrimidin-4-amine (3k). To a suspension of 2,4-dichloropyrimidine 2 ( $1.0 \mathrm{mmol}, 1.0$ equiv) in DCM $(3.0 \mathrm{~mL}, 0.33 \mathrm{M})$ under a nitrogen atmosphere was added $(R)$-(-)-2-aminobutane ( $2.0 \mathrm{mmol}, 2.0$ equiv) at rt. After stirring for $6 \mathrm{~h}$, the reaction mixture was concentrated. Purification using automated flash chromatography (EtOAc/ hexanes) was followed by evaporation, giving $3 \mathbf{k}$ as a colorless oil (0.071 g, 38\%). TLC $R_{f} 0.25$ (30\% EtOAc/hexanes). LC-MS (ESI) $m / z: 188[\mathrm{M}+\mathrm{H}]^{+} .[\alpha]_{\mathrm{D}}{ }^{20}=-45\left(c 0.00048, \mathrm{CHCl}_{3}\right) .{ }^{1} \mathrm{H}$ $\operatorname{NMR}(500 \mathrm{MHz}, \mathrm{MeOD}) \delta 7.79(\mathrm{~d}, J=6.1 \mathrm{~Hz}, 1 \mathrm{H}), 6.37(\mathrm{~d}, J=$ $6.7 \mathrm{~Hz}, 1 \mathrm{H}), 4.17-3.96(\mathrm{~m}, 1 \mathrm{H}), 1.65-1.48(\mathrm{~m}, 2 \mathrm{H}), 1.19(\mathrm{~d}, J=$ $6.6 \mathrm{~Hz}, 3 \mathrm{H}), 0.96(\mathrm{t}, J=7.4 \mathrm{~Hz}, 3 \mathrm{H}) .{ }^{13} \mathrm{C}$ NMR $(126 \mathrm{MHz}$, MeOD) $\delta 163.47,160.19,153.91,104.38,48.21,28.81,18.74$, 9.37.

(R)- $N^{4}$-(sec-Butyl)- $N^{2}-(3-($ pyridin-3-yl)phenyl)pyrimidine2,4 -diamine $(5 \mathrm{k})$. To a mixture of $3 \mathrm{k}(0.10 \mathrm{mmol}, 1.0$ equiv $)$ in $\mathrm{EtOH}(0.50 \mathrm{~mL}, 0.20 \mathrm{M})$ under a nitrogen atmosphere at rt were added 3-(pyridin-3-yl)aniline ( $0.11 \mathrm{mmol}, 1.1$ equiv) and a drop of $1 \mathrm{~N} \mathrm{HCl}$. The reaction mixture was heated to reflux and stirred at that temperature for $2 \mathrm{~h}$; it was then cooled to rt. Purification using automated flash chromatography $(\mathrm{MeOH} / \mathrm{DCM})$ was followed by evaporation, giving 5k as a colorless oil (0.003 g, 9\%). TLC $R_{f} 0.3$ (80\% EtOAc/hexanes). LC-MS (ESI) $m / z$ : $320[\mathrm{M}+\mathrm{H}]^{+} .[\alpha]_{\mathrm{D}}{ }^{20}=-10\left(c 0.000033, \mathrm{CHCl}_{3}\right) .{ }^{1} \mathrm{H} \mathrm{NMR}$ $\left(500 \mathrm{MHz}, \mathrm{CDCl}_{3}\right) \delta 8.87(\mathrm{~s}, 1 \mathrm{H}), 8.59(\mathrm{~s}, 1 \mathrm{H}), 7.96(\mathrm{~s}, 2 \mathrm{H})$, $7.89(\mathrm{dt}, J=7.8,1.8 \mathrm{~Hz}, 1 \mathrm{H}), 7.57(\mathrm{ddd}, J=8.1,2.0,1.0 \mathrm{~Hz}, 1 \mathrm{H})$, $7.42-7.32(\mathrm{~m}, 2 \mathrm{H}), 7.22-7.15(\mathrm{~m}, 2 \mathrm{H}), 5.85(\mathrm{~d}, J=5.6 \mathrm{~Hz}, 1 \mathrm{H})$, 4.64 (br s, $1 \mathrm{H}), 3.86$ (br s, $1 \mathrm{H}), 1.66-1.47(\mathrm{~m}, 2 \mathrm{H}), 1.21(\mathrm{~d}, J=$ $6.5 \mathrm{~Hz}, 3 \mathrm{H}), 0.93(\mathrm{t}, J=7.4 \mathrm{~Hz}, 3 \mathrm{H}) .{ }^{13} \mathrm{C} \mathrm{NMR}(126 \mathrm{MHz}$, 
$\left.\mathrm{CDCl}_{3}\right) \delta 162.43,159.67,155.70,148.44,148.42,140.87,138.49$, $136.94,134.44,129.44,123.46,120.63,118.72,117.72,96.94$, 48.20, 29.69, 20.30, 10.36 .

2-Chloro-N-cyclopropylpyrimidin-4-amine (3l). A mixture of 2,4-dichloropyrimidine 2 (10 mmol, 1.0 equiv) and cyclopropylamine ( $10 \mathrm{mmol}, 1.0$ equiv) was stirred at rt in DCM (20 $\mathrm{mL}, 0.50 \mathrm{M})$ in a nitrogen atmosphere. After stirring for $16 \mathrm{~h}$, the reaction mixture was concentrated to remove volatiles. Purification using automated flash chromatography (EtOAc/ hexanes) was followed by evaporation, giving 31 as a white solid (0.39 g, 23\%). TLC $R_{f} 0.3$ (40\% EtOAc/hexanes). LC-MS (ESI) $m / z: 172[\mathrm{M}+\mathrm{H}]^{+} .{ }^{1} \mathrm{H}$ NMR $\left(500 \mathrm{MHz}, \mathrm{CDCl}_{3}\right) \delta 8.15$ $(\mathrm{d}, J=5.9 \mathrm{~Hz}, 1 \mathrm{H}), 6.77-6.48(\mathrm{~m}, 1 \mathrm{H}), 5.74(\mathrm{br} \mathrm{s}, 1 \mathrm{H}), 2.59(\mathrm{br}$ s, $1 \mathrm{H}), 1.00-0.83(\mathrm{~m}, 2 \mathrm{H}), 0.72-0.54(\mathrm{~m}, 2 \mathrm{H}) .{ }^{13} \mathrm{C}$ NMR (126 $\left.\mathrm{MHz}, \mathrm{CDCl}_{3}\right) \delta 165.25,160.30,157.64,101.64,23.46,7.71$.

$N^{4}$-Cyclopropyl- $N^{2}-(3-($ pyridin-3-yl)phenyl)pyrimidine-2,4diamine (5l). To a mixture of 31 ( $0.10 \mathrm{mmol}, 1.0$ equiv $)$ in $\mathrm{EtOH}$ $(0.50 \mathrm{~mL}, 0.20 \mathrm{M})$ under a nitrogen atmosphere at $\mathrm{rt}$ were added 3-(pyridin-3-yl)aniline ( $0.11 \mathrm{mmol}, 1.1$ equiv) and a drop of $1 \mathrm{~N}$ $\mathrm{HCl}$. The reaction mixture was heated to reflux and stirred at that temperature for $3 \mathrm{~h}$; it was then cooled to rt. Purification using automated flash chromatography $(\mathrm{MeOH} / \mathrm{DCM})$ was followed by evaporation, giving 51 as a colorless oil $(0.004 \mathrm{~g}, 13 \%)$. TLC $R_{f}$ 0.2 (5\% MeOH/DCM). LC-MS (ESI) $m / z: 304[\mathrm{M}+\mathrm{H}]^{+} .{ }^{1} \mathrm{H}$ $\operatorname{NMR}\left(500 \mathrm{MHz}, \mathrm{CDCl}_{3}\right) \delta 8.87(\mathrm{~d}, J=2.6 \mathrm{~Hz}, 1 \mathrm{H}), 8.58(\mathrm{~d}, J=$ $5.0 \mathrm{~Hz}, 1 \mathrm{H}), 8.05(\mathrm{~d}, J=5.0 \mathrm{~Hz}, 1 \mathrm{H}), 7.93(\mathrm{~s}, 1 \mathrm{H}), 7.86-7.88(\mathrm{~m}$ $1 \mathrm{H}), 7.56(\mathrm{~d}, J=8.3 \mathrm{~Hz}, 1 \mathrm{H}), 7.41-7.31(\mathrm{~m}, 2 \mathrm{H}), 7.19(\mathrm{~d}, J=7.6$ $\mathrm{Hz}, 1 \mathrm{H}), 7.13(\mathrm{~s}, 1 \mathrm{H}), 6.32-6.08(\mathrm{~m}, 1 \mathrm{H}), 5.22(\mathrm{~s}, 1 \mathrm{H}), 2.57(\mathrm{br}$ $\mathrm{s}, 1 \mathrm{H}), 0.84-0.78(\mathrm{~m}, 2 \mathrm{H}), 0.62-0.56(\mathrm{~m}, 2 \mathrm{H}) .{ }^{13} \mathrm{C}$ NMR $(126$ $\left.\mathrm{MHz} \mathrm{CDCl}_{3}\right) \delta 164.36,159.65,156.84,148.46,148.43,140.76$, 138.48, 136.86, 134.44, 129.45, 123.49, 120.73, 118.81, 117.77, 95.85, 23.46, 7.59.

2-Chloro-N-(1-(trifluoromethyl)cyclopropyl)pyrimidin-4amine $(3 \mathrm{~m})$. A mixture of 2,4-dichloropyrimidine $2(2.0 \mathrm{mmol}$, 1.0 equiv) and 1-(trifluoromethyl)cyclopropanamine $(2.5 \mathrm{mmol}$, 1.25 equiv) was stirred in NMP $(1.0 \mathrm{~mL}, 2.0 \mathrm{M})$ under microwave irradiation at $140{ }^{\circ} \mathrm{C}$ and $24 \mathrm{~W}$ for $1 \mathrm{~h}$. Purification using automated flash chromatography (EtOAc/hexanes) was followed by evaporation, giving $3 \mathrm{~m}$ as a white solid (0.013 g, 3\%). TLC $R_{f} 0.4$ (30\% EtOAc/hexanes). LC-MS (ESI) $m / z: 238[\mathrm{M}$ $+\mathrm{H}]^{+}{ }^{1} \mathrm{H}$ NMR $\left(500 \mathrm{MHz}, \mathrm{CDCl}_{3}\right) \delta 8.24(\mathrm{~d}, J=5.8 \mathrm{~Hz}, 1 \mathrm{H})$, $6.68(\mathrm{~d}, J=5.8 \mathrm{~Hz}, 1 \mathrm{H}), 5.85($ br s, $1 \mathrm{H}), 1.56-1.45(\mathrm{~m}, 2 \mathrm{H})$, 1.17-1.19 (m, 2H). $\left.{ }^{13} \mathrm{C} \mathrm{NMR} \mathrm{(126} \mathrm{MHz,} \mathrm{CDCl}_{3}\right) \delta 164.16$, $160.60,158.41,125.15$ (q, $\left.J=277 \mathrm{~Hz}, \mathrm{CF}_{3}\right), 119.37,102.39$, 34.46, 34.16, 33.86, 12.67, 12.66, 12.64. ${ }^{19} \mathrm{~F}$ NMR (471 MHz, $\left.\mathrm{CDCl}_{3}\right) \delta-73.26$.

$N^{2}$-(3-(Pyridin-3-yl)phenyl)- $N^{4}-(1-$ (trifluoromethyl)cyclopropyl)pyrimidine-2,4-diamine $(5 \mathrm{~m})$. To a mixture of $3 \mathrm{~m}$ (0.038 mmol, 1.0 equiv) in $\mathrm{EtOH}(0.50 \mathrm{~mL}, 0.76 \mathrm{M})$ under a nitrogen atmosphere at $\mathrm{rt}$ was added 3-(pyridin-3-yl)aniline ( $0.045 \mathrm{mmol}, 1.2$ equiv). The reaction mixture was heated to reflux and stirred at that temperature for $3 \mathrm{~h}$; it was then cooled to rt. Purification using automated flash chromatography $(\mathrm{MeOH} /$ DCM) was followed by evaporation, giving $5 \mathrm{~m}$ as a colorless oil (0.002 g, 14\%). TLC $R_{f} 0.5$ (10\% MeOH/DCM). LC-MS (ESI) $m / z: 372[\mathrm{M}+\mathrm{H}]^{+} .{ }^{1} \mathrm{H} \mathrm{NMR}\left(500 \mathrm{MHz}, \mathrm{CDCl}_{3}\right) \delta 8.88(\mathrm{~s}, 1 \mathrm{H})$, $8.61(\mathrm{~s}, 1 \mathrm{H}), 8.10(\mathrm{~d}, J=5.8 \mathrm{~Hz}, 1 \mathrm{H}), 7.95-7.82(\mathrm{~m}, 2 \mathrm{H}), 7.56-$ $7.58(\mathrm{~m}, 1 \mathrm{H}), 7.41(\mathrm{t}, J=7.9 \mathrm{~Hz}, 2 \mathrm{H}), 7.25-7.18(\mathrm{~m}, 1 \mathrm{H}), 6.99$ $(\mathrm{s}, 1 \mathrm{H}), 6.21(\mathrm{~d}, J=5.6 \mathrm{~Hz}, 1 \mathrm{H}), 5.35(\mathrm{~s}, 1 \mathrm{H}), 1.45-1.36(\mathrm{~m}$, $2 \mathrm{H}), 1.22-1.12(\mathrm{~m}, 2 \mathrm{H}) .{ }^{13} \mathrm{C}$ NMR $\left(126 \mathrm{MHz}, \mathrm{CDCl}_{3}\right) \delta$ $163.00,159.67,157.42,148.46,140.39,138.63,134.45,129.50$, $125.46\left(\mathrm{q}, J=275.94, \mathrm{CF}_{3}\right), 122.16,121.12,118.96,117.91$,
96.47, 34.56, 34.25, 33.95, 33.65, 12.74. ${ }^{19} \mathrm{~F}$ NMR (471 MHz, $\left.\mathrm{CDCl}_{3}\right) \delta-73.18$.

2-Chloro-N-cyclobutylpyrimidin-4-amine (3n). A mixture of 2,4-dichloropyrimidine 2 (10 mmol, 1.0 equiv) and cyclobutylamine (10 mmol, 1.0 equiv) was stirred at $\mathrm{rt}$ in DCM $(20 \mathrm{~mL}$, $0.50 \mathrm{M})$ in a nitrogen atmosphere. After stirring for $16 \mathrm{~h}$, the reaction mixture was concentrated to remove volatiles. Purification using automated flash chromatography (EtOAc/ hexanes) was followed by evaporation, giving $3 \mathbf{n}$ as a white solid (0.059 g, 3\%). TLC $R_{f} 0.3$ (40\% EtOAc/hexanes). LC-MS (ESI) $m / z: 184[\mathrm{M}+\mathrm{H}]^{+} .{ }^{1} \mathrm{H}$ NMR $\left(500 \mathrm{MHz}, \mathrm{CDCl}_{3}\right) \delta 8.04$ $(\mathrm{d}, J=10.9 \mathrm{~Hz}, 1 \mathrm{H}), 6.21(\mathrm{~d}, J=10.0 \mathrm{~Hz}, 1 \mathrm{H}), 5.48(\mathrm{br} \mathrm{s}, 1 \mathrm{H})$, 4.01 (br s, 1H), 2.57-2.40 (m, 2H), 1.92-1.97 (m, 2H), 1.90$1.74(\mathrm{~m}, 2 \mathrm{H}) .{ }^{13} \mathrm{C} \mathrm{NMR}\left(126 \mathrm{MHz}, \mathrm{CDCl}_{3}\right) \delta 162.57,160.51$, 157.78, 100.35, 46.73, 31.27, 30.75, 15.16 .

$N^{4}$-Cyclobutyl- $N^{2}$-(3-(pyridin-3-yl)phenyl)pyrimidine-2,4diamine (5n). To a mixture of $3 \mathrm{n}(0.10 \mathrm{mmol}, 1.0$ equiv) in $\mathrm{EtOH}(0.50 \mathrm{~mL}, 0.20 \mathrm{M})$ under a nitrogen atmosphere at rt were added 3-(pyridin-3-yl)aniline ( $0.11 \mathrm{mmol}, 1.1$ equiv) and a drop of $1 \mathrm{~N} \mathrm{HCl}$. The reaction mixture was heated to reflux and stirred at that temperature for $3 \mathrm{~h}$; it was then cooled to rt. Purification using automated flash chromatography ( $\mathrm{MeOH} / \mathrm{DCM})$ was followed by evaporation, giving $5 \mathrm{n}$ as a yellow oil (0.002 g, 6\%). TLC $R_{f} 0.1$ (5\% MeOH/DCM). LC-MS (ESI) $m / z: 318[\mathrm{M}+$ $\mathrm{H}]^{+} .{ }^{1} \mathrm{H} \mathrm{NMR}(500 \mathrm{MHz}, \mathrm{MeOD}) \delta 8.85(\mathrm{~s}, 1 \mathrm{H}), 8.62-8.49(\mathrm{~m}$, $1 \mathrm{H}), 8.35-8.14(\mathrm{~m}, 1 \mathrm{H}), 8.13(\mathrm{dt}, J=8.0,1.9 \mathrm{~Hz}, 1 \mathrm{H}), 7.77(\mathrm{~s}$, $1 \mathrm{H}), 7.54-7.60(\mathrm{~m}, 2 \mathrm{H}), 7.42(\mathrm{t}, J=7.9 \mathrm{~Hz}, 1 \mathrm{H}), 7.26(\mathrm{~d}, J=7.6$, $1 \mathrm{H}), 5.94(\mathrm{~d}, J=5.9 \mathrm{~Hz}, 1 \mathrm{H}), 4.40-4.56(\mathrm{~m}, 1 \mathrm{H}), 2.42-2.25(\mathrm{~m}$, $2 \mathrm{H}), 1.92-2.01(\mathrm{~m}, 2 \mathrm{H}), 1.79-1.54(\mathrm{~m}, 2 \mathrm{H}) .{ }^{13} \mathrm{C}$ NMR $(126$ $\mathrm{MHz}, \mathrm{MeOD}) \delta 162.15,159.43,153.31,147.28,147.04,141.37$, $137.82,137.63$, 135.20, 129.03, 124.01, 120.00, 118.93, 117.64, 97.37, 45.88, 30.32, 14.57 .

2-Chloro- $N$-cyclopentylpyrimidin-4-amine (3o). A mixture of 2,4-dichloropyrimidine 2 (10 mmol, 1.0 equiv) and cyclopentylamine ( $20 \mathrm{mmol}, 2.0$ equiv) was stirred at $\mathrm{rt}$ in DCM (20 $\mathrm{mL}, 0.50 \mathrm{M})$ in a nitrogen atmosphere. After $16 \mathrm{~h}$, the reaction mixture was concentrated. Purification using automated flash chromatography (EtOAc/hexanes) followed by evaporation gave $3 \mathrm{o}$ as a yellow oil $(1.12 \mathrm{~g}, 57 \%)$. TLC $R_{f} 0.25(40 \%$ EtOAc/hexanes). LC-MS (ESI) $m / z: 200[\mathrm{M}+\mathrm{H}]^{+} .{ }^{1} \mathrm{H}$ NMR $\left(500 \mathrm{MHz}, \mathrm{CDCl}_{3}\right) \delta 8.03(\mathrm{~d}, J=11.7 \mathrm{~Hz}, 1 \mathrm{H}), 6.32(\mathrm{~d}, J=6.3$ $\mathrm{Hz}, 1 \mathrm{H}), 5.46$ (br s, $1 \mathrm{H}), 3.93$ (br s, $1 \mathrm{H}), 2.19-1.94(\mathrm{~m}, 2 \mathrm{H})$, $1.87-1.63(\mathrm{~m}, 4 \mathrm{H}), 1.51-1.56(\mathrm{~m}, 2 \mathrm{H}) .{ }^{13} \mathrm{C} \mathrm{NMR}(126 \mathrm{MHz}$, $\left.\mathrm{CDCl}_{3}\right) \delta 163.22,160.56,157.25,100.46,53.12$, 33.08, 23.66.

$N^{4}$-Cyclopentyl- $N^{2}$-(3-(pyridin-3-yl)phenyl)pyrimidine-2,4diamine (5o). To a mixture of $30(0.10 \mathrm{mmol}, 1.0$ equiv) in $\mathrm{EtOH}(0.50 \mathrm{~mL}, 0.20 \mathrm{M})$ under a nitrogen atmosphere at rt were added 3-(pyridin-3-yl)aniline ( $0.11 \mathrm{mmol}, 1.1$ equiv) and a drop of $1 \mathrm{~N} \mathrm{HCl}$. The reaction mixture was heated to reflux and stirred at that temperature for $3 \mathrm{~h}$; it was then cooled to rt. Purification using automated flash chromatography $(\mathrm{MeOH} / \mathrm{DCM})$ was followed by evaporation, giving 50 as a white oil $(0.005 \mathrm{~g}, 15 \%)$. TLC $R_{f} 0.5$ (10\% MeOH/DCM). LC-MS (ESI) $m / z: 332[\mathrm{M}+$ $\mathrm{H}]^{+} .{ }^{1} \mathrm{H} \mathrm{NMR}(400 \mathrm{MHz}, \mathrm{MeOD}) \delta 8.81(\mathrm{~s}, 1 \mathrm{H}), 8.53(\mathrm{~d}, J=5.1$ $\mathrm{Hz}, 1 \mathrm{H}), 8.21$ (br s, $1 \mathrm{H}), 8.09$ (d, $J=7.5 \mathrm{~Hz}, 1 \mathrm{H}), 7.74(\mathrm{~s}, 1 \mathrm{H})$, $7.67-7.56(\mathrm{~m}, 1 \mathrm{H}), 7.51-7.54(\mathrm{~m}, 1 \mathrm{H}), 7.40(\mathrm{t}, J=7.9 \mathrm{~Hz}, 1 \mathrm{H})$, $7.23(\mathrm{dd}, J=7.6,1.9 \mathrm{~Hz}, 1 \mathrm{H}), 5.96(\mathrm{~d}, J=5.8 \mathrm{~Hz}, 1 \mathrm{H}), 4.30(\mathrm{~s}$, $1 \mathrm{H}), 1.96-2.01(\mathrm{~m}, 2 \mathrm{H}), 1.68-1.75(\mathrm{~m}, 2 \mathrm{H}), 1.45-1.53(\mathrm{~m}$, $4 \mathrm{H}) .{ }^{13} \mathrm{C}$ NMR (101 MHz, MeOD) $\delta 164.22,161.02,148.64$, $148.46,142.94,139.27,139.02,136.62,130.40,125.39,121.27$, $120.25,118.99,53.54,33.77,24.70$.

$\mathrm{N}$-(2-Chloropyrimidin-4-yl)-O-methylhydroxylamine (3p). A mixture of 2,4-dichloropyrimidine 2 (10 mmol, 1.0 equiv), 
$O$-methylhydroxylamine $\mathrm{HCl}$ (15 mmol, 1.5 equiv), and triethylamine $(20 \mathrm{mmol}, 2.0$ equiv) was stirred at $\mathrm{rt}$ in a 0.50 $\mathrm{M}$ mixture of $\mathrm{DCM}(10 \mathrm{~mL})$ and $\mathrm{MeOH}(10 \mathrm{~mL})$ under a nitrogen atmosphere for $16 \mathrm{~h}$. The reaction mixture was then concentrated to remove volatiles. To the crude residue was added water $(10 \mathrm{~mL})$, and then, the crude was extracted into EtOAc $(3 \times 10 \mathrm{~mL})$. The organic phase was dried over magnesium sulfate, filtered, and concentrated. Purification using automated flash chromatography (EtOAc/hexanes) was followed by evaporation, giving $3 \mathrm{p}$ as a white solid $(0.19 \mathrm{~g}, 12 \%)$. TLC $R_{f} 0.5$ (50\% EtOAc/hexanes). LC-MS (ESI) $m / z: 162[\mathrm{M}$ $+\mathrm{H}]^{+} .{ }^{1} \mathrm{H}$ NMR $\left(500 \mathrm{MHz}, \mathrm{CDCl}_{3}\right) \delta 8.47(\mathrm{br} \mathrm{s}, 1 \mathrm{H}), 8.31$ (d, $=5.7 \mathrm{~Hz}, 1 \mathrm{H}), 6.78(\mathrm{~d}, J=5.8 \mathrm{~Hz}, 1 \mathrm{H}), 3.84(\mathrm{~s}, 3 \mathrm{H}) .{ }^{13} \mathrm{C} \mathrm{NMR}$ $\left(126 \mathrm{MHz} \mathrm{CDCl}_{3}\right) \delta 166.53,159.99,158.94,101.72,64.25$.

4-(Methoxyamino)-N-(3-(pyridin-3-yl)phenyl)pyrimidin-2amine (5p). To a mixture of $3 \mathbf{p}(0.10 \mathrm{mmol}, 1.0$ equiv $)$ in $\mathrm{EtOH}$ $(0.50 \mathrm{~mL}, 0.20 \mathrm{M})$ under a nitrogen atmosphere at $\mathrm{rt}$ were added 3-(pyridin-3-yl)aniline ( $0.11 \mathrm{mmol}, 1.1$ equiv) and a drop of $1 \mathrm{~N}$ $\mathrm{HCl}$. The reaction mixture was heated to reflux and stirred at that temperature for $3 \mathrm{~h}$; it was then cooled to rt. Purification using automated flash chromatography (MeOH/DCM) was followed by evaporation, giving $\mathbf{5 p}$ as a colorless oil $(0.006 \mathrm{~g}, 20 \%)$. TLC $R_{f} 0.4$ (5\% MeOH/DCM). LC-MS (ESI) $m / z: 294[\mathrm{M}+\mathrm{H}]^{+}$. ${ }^{1} \mathrm{H} \mathrm{NMR}\left(500 \mathrm{MHz}, \mathrm{CDCl}_{3}\right) \delta 8.90(\mathrm{~s}, 1 \mathrm{H}), 8.63(\mathrm{~d}, J=5.0 \mathrm{~Hz}$, $1 \mathrm{H}), 8.24(\mathrm{~d}, J=5.6 \mathrm{~Hz}, 1 \mathrm{H}), 7.98-7.86(\mathrm{~m}, 2 \mathrm{H}), 7.61(\mathrm{~s}, 1 \mathrm{H})$, $7.56(\mathrm{~d}, J=8.2 \mathrm{~Hz}, 1 \mathrm{H}), 7.46-7.34(\mathrm{~m}, 2 \mathrm{H}), 7.27-7.21(\mathrm{~m}, 1 \mathrm{H})$, $7.18-7.09(\mathrm{~m}, 1 \mathrm{H}), 6.40(\mathrm{~d}, J=5.6 \mathrm{~Hz}, 1 \mathrm{H}), 3.83(\mathrm{~s}, 3 \mathrm{H}) .{ }^{13} \mathrm{C}$ NMR $\left(126 \mathrm{MHz}, \mathrm{CDCl}_{3}\right) \delta 166.38,159.30,158.40,148.49$, $148.41,140.30,138.54,136.70,134.44,129.54,123.53,121.14$, $118.95,117.94,95.89,64.15$.

tert-Butyl 4-((2-chloropyrimidin-4-yl)amino)piperidine-1carboxylate (3q). To a suspension of 2,4-dichloropyrimidine 2 ( $5.0 \mathrm{mmol}, 1.0$ equiv) in DCM $(10 \mathrm{~mL}, 0.50 \mathrm{M})$ under a nitrogen atmosphere was added 1-Boc-4-aminopiperidine (7.5 mmol, 1.5 equiv) at rt. After stirring for $5 \mathrm{~h}$ the reaction mixture was concentrated. Purification using automated flash chromatography (EtOAc/hexanes) was followed by evaporation, giving 3q as a white foam $(0.41 \mathrm{~g}, 26 \%)$. TLC $R_{f} 0.10$ (30\% EtOAc/ hexanes). LC-MS (ESI) $m / z: 313[\mathrm{M}+\mathrm{H}]^{+} .{ }^{1} \mathrm{H}$ NMR (400 $\mathrm{MHz}, \mathrm{MeOD}) \delta 7.85$ (br s, $1 \mathrm{H}), 6.40$ (br s, $1 \mathrm{H}), 4.03-4.13(\mathrm{~m}$, $3 \mathrm{H}), 2.99$ (s, 2H), 2.06-1.89 (m, 2H), 1.48 (s, 9H), 1.45-1.35 $(\mathrm{m}, 2 \mathrm{H}) .{ }^{13} \mathrm{C}$ NMR (101 MHz, MeOD) $\delta 164.55,161.59$, 156.45, 155.77, 105.96, 101.41, 81.17, 43.88, 32.45, 28.68.

tert-Butyl 4-((2-((3-(pyridin-3-yl)phenyl)amino)pyrimidin4-yl)amino)piperidine-1-carboxylate (3r). To a mixture of $3 q$ $(0.10 \mathrm{mmol}, 1.0$ equiv) in $\mathrm{EtOH}(0.50 \mathrm{~mL}, 0.20 \mathrm{M})$ under a nitrogen atmosphere at $\mathrm{rt}$ were added 3-(pyridin-3-yl)aniline $(0.11 \mathrm{mmol}, 1.1$ equiv) and a drop of $1 \mathrm{~N} \mathrm{HCl}$. The reaction mixture was heated to reflux and stirred at that temperature for 3 $\mathrm{h}$; it was then cooled to rt. Purification using automated flash chromatography (MeOH/DCM) was followed by evaporation, giving $3 \mathbf{r}$ as a colorless oil $(0.007 \mathrm{~g}, 16 \%)$. TLC $R_{f} 0.5(10 \%$ $\mathrm{MeOH} / \mathrm{DCM})$. LC-MS (ESI) $m / z: 447[\mathrm{M}+\mathrm{H}]^{+} .{ }^{1} \mathrm{H}$ NMR $(500 \mathrm{MHz}, \mathrm{MeOD}) \delta 8.82(\mathrm{~s}, 1 \mathrm{H}), 8.53(\mathrm{~d}, J=5.0 \mathrm{~Hz}, 1 \mathrm{H})$, 8.19-8.05 (m, 2H), $7.77(\mathrm{~s}, 1 \mathrm{H}), 7.59(\mathrm{~d}, J=7.4 \mathrm{~Hz}, 1 \mathrm{H}), 7.54$ $(\mathrm{dd}, J=7.9,4.9 \mathrm{~Hz}, 1 \mathrm{H}), 7.42(\mathrm{t}, J=7.9 \mathrm{~Hz}, 1 \mathrm{H}), 7.31-7.16(\mathrm{~m}$, $1 \mathrm{H}), 5.97(\mathrm{~d}, J=5.9 \mathrm{~Hz}, 1 \mathrm{H}), 4.02(\mathrm{~s}, 1 \mathrm{H}), 3.98-3.84(\mathrm{~m}, 2 \mathrm{H})$, $2.60-2.69(\mathrm{~m}, 2 \mathrm{H}), 1.99-1.90(\mathrm{~m}, 2 \mathrm{H}), 1.47(\mathrm{~s}, 9 \mathrm{H}), 1.40-1.28$ $(\mathrm{m}, 2 \mathrm{H}) .{ }^{13} \mathrm{C}$ NMR $(126 \mathrm{MHz}, \mathrm{MeOD}) \delta 162.35,159.45$, $154.98,153.44,147.35,147.11,141.31,137.87,137.60,135.29$, $129.05,124.10,120.17,119.14,117.92,97.70,79.69,53.40$, $42.05,31.26,27.27$.
$N^{4}$-(Piperidin-4-yl)- $N^{2}$-(3-(pyridin-3-yl)phenyl)pyrimidine2,4-diamine (5q). To $3 \mathbf{r}$ (0.020 mmol, 1.0 equiv) in DCM (1.0 $\mathrm{mL}, 0.20 \mathrm{M})$ under a nitrogen atmosphere at rt was added trifluoroacetic acid ( $0.20 \mathrm{mmol}, 10$ equiv). The reaction mixture was stirred at $\mathrm{rt}$ for $3 \mathrm{~h}$ and then concentrated. Purification using automated reversed-phase flash chromatography (water/ $\mathrm{MeOH})$ was followed by evaporation, giving $\mathbf{5 q}$ as a colorless oil (0.007 g, 100\%). LC-MS (ESI) $\mathrm{m} / z: 347[\mathrm{M}+\mathrm{H}]^{+} .{ }^{1} \mathrm{H}$ $\operatorname{NMR}(500 \mathrm{MHz}, \mathrm{MeOD}) \delta 8.91(\mathrm{~s}, 1 \mathrm{H}), 8.63(\mathrm{~s}, 1 \mathrm{H}), 8.16(\mathrm{~d}, J$ $=7.9 \mathrm{~Hz}, 1 \mathrm{H}), 8.02(\mathrm{~s}, 1 \mathrm{H}), 7.80(\mathrm{~s}, 1 \mathrm{H}), 7.61(\mathrm{~d}, J=8.3 \mathrm{~Hz}, 2 \mathrm{H})$, $7.52(\mathrm{t}, J=7.8 \mathrm{~Hz}, 1 \mathrm{H}), 7.43(\mathrm{~d}, J=7.7 \mathrm{~Hz}, 1 \mathrm{H}), 6.15(\mathrm{~d}, J=6.2$ $\mathrm{Hz}, 1 \mathrm{H}), 4.16-4.20(\mathrm{~m}, 1 \mathrm{H}), 3.35-3.39(\mathrm{~m}, 2 \mathrm{H}), 3.00-2.81(\mathrm{~m}$, $2 \mathrm{H}), 2.22-2.26(\mathrm{~m}, 2 \mathrm{H}), 1.84-1.70(\mathrm{~m}, 2 \mathrm{H}) .{ }^{13} \mathrm{C}$ NMR $(126$ $\mathrm{MHz}, \mathrm{MeOD}) \delta 165.13,162.56,161.59,156.29,147.68,147.00$, $139.44,137.92$, 135.19, 129.43, 124.45, 122.05, 120.74, 119.38, 98.25, 45.38, 42.46, 27.85 .

2-Chloro-N-(tetrahydro-2H-pyran-4-yl)pyrimidin-4-amine (3s). To a suspension of 2,4-dichloropyrimidine 2 ( $2.5 \mathrm{mmol}, 1.0$ equiv) in DCM $(5.0 \mathrm{~mL}, 0.50 \mathrm{M})$ under a nitrogen atmosphere was added 4-aminotetrohydropyran ( $5.0 \mathrm{mmol}, 2.0$ equiv) at rt. After stirring for $16 \mathrm{~h}$, the reaction mixture was concentrated. Purification using automated flash chromatography (EtOAc/ hexanes) was followed by evaporation, giving $3 \mathrm{~s}$ as a white solid (0.19 g, 35\%). TLC $R_{f} 0.30$ (70\% EtOAc/hexanes). LC-MS (ESI) $m / z: 216[\mathrm{M}+\mathrm{H}]^{+} .{ }^{1} \mathrm{H}$ NMR $\left(500 \mathrm{MHz}, \mathrm{CDCl}_{3}\right) \delta 8.12-$ $7.94(\mathrm{~m}, 1 \mathrm{H}), 6.25(\mathrm{~d}, J=5.9 \mathrm{~Hz}, 1 \mathrm{H}), 5.01(\mathrm{br} \mathrm{s}, 1 \mathrm{H}), 4.00-4.04$ $(\mathrm{m}, 2 \mathrm{H}), 3.53-3.59(\mathrm{~m}, 2 \mathrm{H}), 2.11-1.96(\mathrm{~m}, 2 \mathrm{H}), 1.63-1.49(\mathrm{~m}$ $2 \mathrm{H}) .{ }^{13} \mathrm{C} \mathrm{NMR}\left(126 \mathrm{MHz}, \mathrm{CDCl}_{3}\right) \delta 162.63,160.99,157.11$, 66.47, 47.24, 32.83.

$N^{2}$-(3-(Pyridin-3-yl)phenyl)- $N^{4}$-(tetrahydro-2H-pyran-4-yl)pyrimidine-2,4-diamine (5r). To a mixture of $3 \mathrm{~s}(0.094 \mathrm{mmol}$, 1.0 equiv) in $\mathrm{EtOH}(0.50 \mathrm{~mL}, 0.19 \mathrm{M})$ under a nitrogen atmosphere at $\mathrm{rt}$ were added 3-(pyridin-3-yl)aniline (0.103 mmol, 1.1 equiv) and a drop of $1 \mathrm{~N} \mathrm{HCl}$. The reaction mixture was heated to reflux and stirred at that temperature for $4 \mathrm{~h}$; it was then cooled to rt. Purification using automated flash chromatography $(\mathrm{MeOH} / \mathrm{DCM})$ was followed by evaporation, giving $\mathbf{5 r}$ as a colorless oil ( $0.005 \mathrm{~g}, 15 \%)$. TLC $R_{f} 0.6(10 \% \mathrm{MeOH} / \mathrm{DCM})$. LC-MS (ESI) $m / z: 348[\mathrm{M}+\mathrm{H}]^{+}$. ${ }^{1} \mathrm{H}$ NMR $(500 \mathrm{MHz}$, $\left.\mathrm{CDCl}_{3}\right) \delta 8.80(\mathrm{~s}, 1 \mathrm{H}), 8.53(\mathrm{~d}, J=5.1 \mathrm{~Hz}, 1 \mathrm{H}), 7.94-7.84(\mathrm{~m}$, $2 \mathrm{H}), 7.82(\mathrm{~d}, J=8.0 \mathrm{~Hz}, 1 \mathrm{H}), 7.53-7.43(\mathrm{~m}, 1 \mathrm{H}), 7.35-7.27(\mathrm{~m}$, $2 \mathrm{H}), 7.23(\mathrm{~s}, 1 \mathrm{H}), 7.13(\mathrm{dd}, J=7.7,1.6 \mathrm{~Hz}, 1 \mathrm{H}), 5.80(\mathrm{~d}, J=5.8$ $\mathrm{Hz}, 1 \mathrm{H}), 4.66(\mathrm{~s}, 1 \mathrm{H}), 3.91($ br s, $1 \mathrm{H}), 3.86(\mathrm{~d}, J=11.5 \mathrm{~Hz}, 2 \mathrm{H})$, $3.28(\mathrm{t}, J=11.7 \mathrm{~Hz}, 2 \mathrm{H}), 1.93(\mathrm{~d}, J=10.0 \mathrm{~Hz}, 2 \mathrm{H}), 1.54-1.35$ $(\mathrm{m}, 2 \mathrm{H}) .{ }^{13} \mathrm{C} \mathrm{NMR}\left(126 \mathrm{MHz}, \mathrm{CDCl}_{3}\right) \delta 161.92,159.68,155.79$, $148.51,148.43,140.74,138.52,136.95,134.46,129.43,123.53$, 120.84, 118.81, 117.87, 97.05, 66.64, 47.19, 33.17.

$N^{2}$-([1,1'-Biphenyl]-2-yl)- $N^{4}$-isopropylpyrimidine-2,4-diamine (6a). To a mixture of $3 \mathrm{f}(0.10 \mathrm{mmol}, 1.0$ equiv) in $\mathrm{EtOH}$ $(0.50 \mathrm{~mL}, 0.20 \mathrm{M})$ under a nitrogen atmosphere at $\mathrm{rt}$ were added 2 -aminobiphenyl ( $0.15 \mathrm{mmol}, 1.5$ equiv) and a drop of $1 \mathrm{~N} \mathrm{HCl}$. The reaction mixture was heated to reflux and stirred at that temperature for $2 \mathrm{~h}$; it was then cooled to rt. Purification using automated flash chromatography $(\mathrm{MeOH} / \mathrm{DCM})$ was followed by evaporation, giving 6a as a white solid $(0.021 \mathrm{~g}, 69 \%)$. TLC $R_{f}$ $0.7(10 \% \mathrm{MeOH} / \mathrm{DCM})$. LC-MS (ESI) $\mathrm{m} / z: 305[\mathrm{M}+\mathrm{H}]^{+} .{ }^{1} \mathrm{H}$ $\mathrm{NMR}\left(400 \mathrm{MHz}, \mathrm{CDCl}_{3}\right) \delta 8.22(\mathrm{~d}, J=8.2 \mathrm{~Hz}, 1 \mathrm{H}), 7.71(\mathrm{~d}, J=$ $5.9 \mathrm{~Hz}, 1 \mathrm{H}), 7.40-7.33(\mathrm{~m}, 4 \mathrm{H}), 7.31-7.24(\mathrm{~m}, 2 \mathrm{H}), 7.17(\mathrm{~d}, J=$ $1.7 \mathrm{~Hz}, 1 \mathrm{H}), 7.03(\mathrm{td}, J=7.4,1.2 \mathrm{~Hz}, 1 \mathrm{H}), 5.73(\mathrm{~d}, J=6.0 \mathrm{~Hz}$, $1 \mathrm{H}), 4.84($ br s, $1 \mathrm{H}), 3.86($ br s, $1 \mathrm{H}), 1.15(\mathrm{~d}, J=6.4 \mathrm{~Hz}, 6 \mathrm{H}) .{ }^{13} \mathrm{C}$ NMR $\left(101 \mathrm{MHz}, \mathrm{CDCl}_{3}\right) \delta 162.03,158.76,138.82,136.31$, $132.73,130.26$, 129.40, 128.90, 127.89, 127.58, 122.79, 121.39, 42.97, 22.72. 
$N^{2}$-(3-(tert-Butyl)phenyl)- $N^{4}$-isopropylpyrimidine-2,4-diamine (6b). To a mixture of $3 \mathrm{f}(0.15 \mathrm{mmol}, 1.0$ equiv) in EtOH $(0.50 \mathrm{~mL}, 0.30 \mathrm{M})$ under a nitrogen atmosphere at rt were added 3-(tert-butyl)aniline ( $0.22 \mathrm{mmol}, 1.5$ equiv) and a drop of $1 \mathrm{~N}$ $\mathrm{HCl}$. The reaction mixture was heated to reflux and stirred at that temperature for $1 \mathrm{~h}$; it was then cooled to rt. Purification using automated flash chromatography $(\mathrm{MeOH} / \mathrm{DCM})$ was followed by evaporation, giving $\mathbf{6 b}$ as a colorless oil $(0.027 \mathrm{~g}, 65 \%)$. TLC $R_{f} 0.6(10 \% \mathrm{MeOH} / \mathrm{DCM})$. LC-MS (ESI) $m / z: 285[\mathrm{M}+\mathrm{H}]^{+}$. ${ }^{1} \mathrm{H} \mathrm{NMR}\left(500 \mathrm{MHz}, \mathrm{CDCl}_{3}\right.$ ) $\delta 8.74(\mathrm{br} \mathrm{s}, 1 \mathrm{H}), 7.63(\mathrm{~d}, J=31.1$ $\mathrm{Hz}, 2 \mathrm{H}), 7.47(\mathrm{~d}, J=8.0 \mathrm{~Hz}, 1 \mathrm{H}), 7.35-7.22(\mathrm{~m}, 2 \mathrm{H}), 7.14(\mathrm{~d}, J=$ $7.9 \mathrm{~Hz}, 1 \mathrm{H}), 6.05(\mathrm{~d}, J=6.5 \mathrm{~Hz}, 1 \mathrm{H}), 4.23(\mathrm{br} \mathrm{s}, 1 \mathrm{H}), 1.35(\mathrm{~d}, J=$ $2.0 \mathrm{~Hz}, 9 \mathrm{H}), 1.30(\mathrm{~d}, J=8.0 \mathrm{~Hz}, 6 \mathrm{H}) .{ }^{13} \mathrm{C}$ NMR $(126 \mathrm{MHz}$, $\left.\mathrm{CDCl}_{3}\right) \delta 161.95,152.02$, 137.97, 128.41, 120.65, 117.70, 117.59, 43.36, 34.77, 31.32, 22.53 .

$N^{2}$-([1, 1'-Biphenyl]-3-yl)- $N^{4}$-isopropylpyrimidine-2,4-diamine $(6 \mathrm{c})$. To a mixture of $3 \mathrm{f}(0.10 \mathrm{mmol}, 1.0$ equiv $)$ in $\mathrm{EtOH}$ $(0.50 \mathrm{~mL}, 0.20 \mathrm{M})$ under a nitrogen atmosphere at $\mathrm{rt}$ were added 3-aminobiphenyl ( $0.15 \mathrm{mmol}, 1.5$ equiv) and a drop of $1 \mathrm{~N} \mathrm{HCl}$. The reaction mixture was heated to reflux and stirred at that temperature for $2 \mathrm{~h}$; it was then cooled to rt. Purification using automated flash chromatography $(\mathrm{MeOH} / \mathrm{DCM})$ was followed by evaporation, giving $6 \mathrm{c}$ as a gray solid $(0.020 \mathrm{~g}, 66 \%)$. TLC $R_{f}$ $0.7(10 \% \mathrm{MeOH} / \mathrm{DCM})$. LC-MS (ESI) $m / z: 305[\mathrm{M}+\mathrm{H}]^{+} .{ }^{1} \mathrm{H}$ NMR (400 MHz, chloroform-d) $\delta 7.81(\mathrm{~d}, J=8.3 \mathrm{~Hz}, 2 \mathrm{H})$, $7.56-7.47(\mathrm{~m}, 3 \mathrm{H}), 7.43(\mathrm{~d}, J=11.1 \mathrm{~Hz}, 1 \mathrm{H}), 7.35(\mathrm{td}, J=8.2$, 7.7, $1.7 \mathrm{~Hz}, 2 \mathrm{H}), 7.31-7.23(\mathrm{~m}, 2 \mathrm{H}), 7.15(\mathrm{dt}, J=7.7,1.5 \mathrm{~Hz}$, $1 \mathrm{H}), 5.76(\mathrm{dd}, J=6.0,1.3 \mathrm{~Hz}, 1 \mathrm{H}), 4.73(\mathrm{br} \mathrm{s}, 1 \mathrm{H}), 4.01$ (br s, $1 \mathrm{H}), 1.17(\mathrm{~d}, J=8.0 \mathrm{~Hz}, 6 \mathrm{H}) .{ }^{13} \mathrm{C} \mathrm{NMR}\left(101 \mathrm{MHz}, \mathrm{CDCl}_{3}\right) \delta$ $162.12,159.25,141.92,141.38,140.27,129.09,128.62,127.23$, $121.03,118.25,118.12,42.89,22.80$.

$N^{4}$-Isopropyl- $N^{2}$-(3-(pyridin-2-yl)phenyl)pyrimidine-2,4-diamine (6d). To a mixture of $3 \mathrm{f}(0.14 \mathrm{mmol}, 1.0$ equiv) in EtOH $(0.50 \mathrm{~mL}, 0.28 \mathrm{M})$ under a nitrogen atmosphere at rt were added 3-(2-pyridyl)aniline ( $0.15 \mathrm{mmol}, 1.1$ equiv) and a drop of $1 \mathrm{~N}$ $\mathrm{HCl}$. The reaction mixture was heated to reflux and stirred at that temperature for $3 \mathrm{~h}$; it was then cooled to rt. Purification using automated flash chromatography $(\mathrm{MeOH} / \mathrm{DCM})$ was followed by evaporation, giving $6 \mathrm{~d}$ as a colorless oil $(0.014 \mathrm{~g}, 33 \%)$. TLC $R_{f} 0.5(10 \% \mathrm{MeOH} / \mathrm{DCM})$. LC-MS (ESI) $m / z: 306[\mathrm{M}+\mathrm{H}]^{+}$. ${ }^{1} \mathrm{H}$ NMR $(400 \mathrm{MHz}, \mathrm{MeOD}) \delta 8.64(\mathrm{~d}, J=4.9 \mathrm{~Hz}, 1 \mathrm{H}), 8.36(\mathrm{~s}$, $1 \mathrm{H}), 7.99-7.83(\mathrm{~m}, 2 \mathrm{H}), 7.67-7.70(\mathrm{~m}, 2 \mathrm{H}), 7.64-7.54(\mathrm{~m}$, $1 \mathrm{H}), 7.49(\mathrm{t}, J=7.9 \mathrm{~Hz}, 1 \mathrm{H}), 7.45-7.31(\mathrm{~m}, 1 \mathrm{H}), 6.09(\mathrm{~d}, J=6.7$ $\mathrm{Hz}, 1 \mathrm{H}), 4.33$ (br s, $1 \mathrm{H}), 1.24(\mathrm{~d}, J=6.5 \mathrm{~Hz}, 6 \mathrm{H}) .{ }^{13} \mathrm{C}$ NMR $(101$ $\mathrm{MHz}, \mathrm{MeOD}) \delta 163.57,158.73,150.33,141.35,140.40,138.91$, $130.44,123.95,123.43,122.73,122.69,120.88,101.41,44.04$, 22.46.

$N^{4}$-Isopropyl-N²-(3'-methoxy-[1, 1'-biphenyl]-3-yl)pyrimidine-2,4-diamine (6e). To a mixture of $3 \mathrm{f}(0.070 \mathrm{mmol}$, 1.0 equiv) in $\mathrm{EtOH}(0.50 \mathrm{~mL}, 0.14 \mathrm{M})$ under a nitrogen atmosphere at $\mathrm{rt}$ were added ( $3^{\prime}$-methoxybiphenyl-3-yl)amine $\mathrm{HCl}$ salt ( $0.084 \mathrm{mmol}, 1.2$ equiv) and a drop of $1 \mathrm{~N} \mathrm{HCl}$. The reaction mixture was heated to reflux and stirred at that temperature for $16 \mathrm{~h}$; it was then cooled to rt. Purification using automated flash chromatography $(\mathrm{MeOH} / \mathrm{DCM})$ was followed by evaporation, giving $6 \mathrm{e}$ as a colorless oil $(0.013 \mathrm{~g}, 56 \%)$. TLC $R_{f}$ 0.2 (50\% EtOAc/hexanes + 1\% MeOH). LC-MS (ESI) $m / z$ : $335[\mathrm{M}+\mathrm{H}]^{+} .{ }^{1} \mathrm{H}$ NMR $\left(400 \mathrm{MHz}, \mathrm{CDCl}_{3}\right) \delta 8.09-7.88(\mathrm{~m}$, $2 \mathrm{H}), 7.74-7.63(\mathrm{~m}, 2 \mathrm{H}), 7.53-7.40(\mathrm{~m}, 2 \mathrm{H}), 7.40-7.27(\mathrm{~m}$, $3 \mathrm{H}), 7.07-6.92(\mathrm{~m}, 1 \mathrm{H}), 5.96(\mathrm{~d}, J=8.0 \mathrm{~Hz}, 1 \mathrm{H}), 4.95(\mathrm{~d}, J=7.8$ $\mathrm{Hz}, 1 \mathrm{H}), 4.21$ (br s, $1 \mathrm{H}), 3.89$ (s, 3H), 1.36 (d, J=8.0 Hz, 6H). ${ }^{13} \mathrm{C} \mathrm{NMR}\left(126 \mathrm{MHz}, \mathrm{CDCl}_{3}\right) \delta 162.10,159.86,159.03,146.68$,
$142.90,141.79,140.14,129.60,129.08,121.13,119.76,118.45$ $118.20,113.08,112.58,96.25,55.31,42.97,22.76$.

Methyl 3'-((4-(isopropylamino)pyrimidin-2-yl)amino)$\left[1,1^{\prime}\right.$-biphenyl]-3-carboxylate (6f). To a mixture of $3 \mathrm{f}(0.12$ mmol, 1.0 equiv) in $\mathrm{MeOH}(0.50 \mathrm{~mL}, 0.23 \mathrm{M})$ under a nitrogen atmosphere at rt were added 3 '-amino-biphenyl-3-carboxylic acid methyl ester $\mathrm{HCl}(0.13 \mathrm{mmol}, 1.1$ equiv) and a drop of $1 \mathrm{~N}$ $\mathrm{HCl}$. The reaction mixture was heated to reflux and stirred at that temperature for $3 \mathrm{~h}$; it was then cooled to rt. The reaction mixture was diluted with EtOAc $(3 \mathrm{~mL})$ and then washed sequentially with $1 \mathrm{~N} \mathrm{HCl}(3 \mathrm{~mL})$ and brine $(3 \mathrm{~mL})$. The organic phase was dried over magnesium sulfate and concentrated. Purification using automated flash chromatography ( $\mathrm{MeOH} /$ DCM) was followed by evaporation, giving $\mathbf{6 f}$ as a white solid (0.015 g, 36\%). TLC $R_{f} 0.6(10 \% \mathrm{MeOH} / \mathrm{DCM})$. LC-MS (ESI) $m / z: 363[\mathrm{M}+\mathrm{H}]^{+} .{ }^{1} \mathrm{H}$ NMR $\left(400 \mathrm{MHz}, \mathrm{CDCl}_{3}\right) \delta 8.30(\mathrm{~d}, J=$ $1.9 \mathrm{~Hz}, 1 \mathrm{H}), 8.05-7.87(\mathrm{~m}, 3 \mathrm{H}), 7.80(\mathrm{dt}, J=8.0,1.4 \mathrm{~Hz}, 1 \mathrm{H})$, $7.62-7.52(\mathrm{~m}, 1 \mathrm{H}), 7.50(\mathrm{t}, J=7.7 \mathrm{~Hz}, 1 \mathrm{H}), 7.38(\mathrm{t}, J=7.9 \mathrm{~Hz}$, $1 \mathrm{H}), 7.26-7.19(\mathrm{~m}, 1 \mathrm{H}), 7.09(\mathrm{~s}, 1 \mathrm{H}), 5.83(\mathrm{~d}, J=5.8 \mathrm{~Hz}, 1 \mathrm{H})$, 4.63 (br s, $1 \mathrm{H}), 4.05$ (br s, $1 \mathrm{H}), 3.95$ (s, 3H), 1.24 (d, $J=6.4 \mathrm{~Hz}$, $6 \mathrm{H}) .{ }^{13} \mathrm{C} \mathrm{NMR}\left(101 \mathrm{MHz}, \mathrm{CDCl}_{3}\right) \delta 167.08,162.14,159.85$, $155.96,141.74,140.80,140.73,131.64,130.58,129.24,128.70$, $128.38,128.31,120.68,118.40,117.81,52.17,42.81,22.82$.

$N^{4}$-Isopropyl-N²-(3-(oxazol-2-yl)phenyl)pyrimidine-2,4-diamine $(6 \mathrm{~g})$. To a mixture of $3 \mathrm{f}(0.10 \mathrm{mmol}, 1.0$ equiv $)$ in $\mathrm{EtOH}$ $(0.50 \mathrm{~mL}, 0.20 \mathrm{M})$ under a nitrogen atmosphere at $\mathrm{rt}$ were added 3-(1,3-oxaxol-2-yl)aniline ( $0.11 \mathrm{mmol}, 1.1$ equiv) and a drop of 1 $\mathrm{N} \mathrm{HCl}$. The reaction mixture was heated to reflux and stirred at that temperature for $3 \mathrm{~h}$; it was then cooled to rt. Purification using automated flash chromatography $(\mathrm{MeOH} / \mathrm{DCM})$ was followed by evaporation, giving $\mathbf{6 g}$ as a colorless oil $(0.005 \mathrm{~g}$, $17 \%)$. TLC $R_{f} 0.5$ (10\% MeOH/DCM). LC-MS (ESI) $\mathrm{m} / z$ : $296[\mathrm{M}+\mathrm{H}]^{+} .{ }^{1} \mathrm{H} \mathrm{NMR}(500 \mathrm{MHz}, \mathrm{MeOD}) \delta 8.66(\mathrm{t}, J=2.0 \mathrm{~Hz}$, $1 \mathrm{H}), 7.99(\mathrm{~d}, J=0.9 \mathrm{~Hz}, 1 \mathrm{H}), 7.88-7.69(\mathrm{~m}, 1 \mathrm{H}), 7.69-7.57(\mathrm{~m}$, $2 \mathrm{H}), 7.40(\mathrm{t}, J=7.9 \mathrm{~Hz}, 1 \mathrm{H}), 7.31(\mathrm{~d}, J=0.9 \mathrm{~Hz}, 1 \mathrm{H}), 5.96(\mathrm{~d}, J=$ $6.0 \mathrm{~Hz}, 1 \mathrm{H}), 4.33$ (br s, $1 \mathrm{H}), 1.27$ (d, $J=6.5 \mathrm{~Hz}, 6 \mathrm{H}) .{ }^{13} \mathrm{C}$ NMR (126 MHz, MeOD) $\delta 162.52,162.41,159.55,153.55,141.51$, $139.19,128.77,127.53,127.10,120.97,118.86,116.51,97.57$, 41.90, 21.44

$N^{4}$-Isopropyl-N2-(3-(thiazol-2-yl)phenyl)pyrimidine-2,4-diamine (6h). To a mixture of $3 \mathrm{f}(0.10 \mathrm{mmol}, 1.0$ equiv $)$ in $\mathrm{EtOH}$ $(0.50 \mathrm{~mL}, 0.20 \mathrm{M})$ under a nitrogen atmosphere at $\mathrm{rt}$ were added 3-(1,3-thiazol-2-yl)aniline ( $0.11 \mathrm{mmol}, 1.1$ equiv) and a drop of 1 $\mathrm{N} \mathrm{HCl}$. The reaction mixture was heated to reflux and stirred at that temperature for $3 \mathrm{~h}$; it was then cooled to rt. Purification using automated flash chromatography ( $\mathrm{MeOH} / \mathrm{DCM})$ was followed by evaporation, giving $\mathbf{6 h}$ as a colorless oil $(0.004 \mathrm{~g}$, $13 \%)$. TLC $R_{f} 0.5$ (10\% MeOH/DCM). LC-MS (ESI) $m / z$ : $312[\mathrm{M}+\mathrm{H}]^{+} .{ }^{1} \mathrm{H}$ NMR $(500 \mathrm{MHz}, \mathrm{MeOD}) \delta 8.53(\mathrm{t}, J=2.1 \mathrm{~Hz}$, $1 \mathrm{H}), 7.87(\mathrm{~d}, J=3.3 \mathrm{~Hz}, 1 \mathrm{H}), 7.83-7.68(\mathrm{~m}, 1 \mathrm{H}), 7.68-7.58(\mathrm{~m}$, $2 \mathrm{H}), 7.54(\mathrm{~d}, J=10.0 \mathrm{~Hz}, 1 \mathrm{H}), 7.38(\mathrm{t}, J=7.9 \mathrm{~Hz}, 1 \mathrm{H}), 5.96(\mathrm{~d}, J$ $=6.0 \mathrm{~Hz}, 1 \mathrm{H}), 4.35(\mathrm{br} \mathrm{s}, 1 \mathrm{H}), 1.25(\mathrm{~d}, J=6.5 \mathrm{~Hz}, 6 \mathrm{H}) .{ }^{13} \mathrm{C} \mathrm{NMR}$ $(126 \mathrm{MHz}, \mathrm{MeOD}) \delta 169.29,162.43,159.55,153.50,142.90$, $141.60,133.43,128.90,120.62,119.18,116.81,97.61,41.85$, 21.44 .

$N^{2}$-(3-(1H-Pyrazol-3-yl)phenyl)- $N^{4}$-isopropylpyrimidine2,4-diamine (6i). To a mixture of $3 \mathrm{f}(0.10 \mathrm{mmol}, 1.0$ equiv $)$ in $\mathrm{EtOH}(0.50 \mathrm{~mL}, 0.20 \mathrm{M})$ under a nitrogen atmosphere at rt were added 3-( $1 H$-pyrazol-3-yl)aniline $(0.11 \mathrm{mmol}, 1.1$ equiv $)$ and a drop of $1 \mathrm{~N} \mathrm{HCl}$. The reaction mixture was heated to reflux and stirred at that temperature for $3 \mathrm{~h}$; it was then cooled to rt. Purification using automated flash chromatography $(\mathrm{MeOH} /$ DCM) was followed by evaporation, giving $6 \mathbf{i}$ as a white oil 
(0.020 g, 68\%). TLC $R_{f} 0.3(10 \% \mathrm{MeOH} / \mathrm{DCM}) . \mathrm{LC}-\mathrm{MS}$ (ESI) $m / z: 295[\mathrm{M}+\mathrm{H}]^{+} .{ }^{1} \mathrm{H}$ NMR $(500 \mathrm{MHz}, \mathrm{MeOD}) \delta 8.12(\mathrm{br} \mathrm{s}$, $1 \mathrm{H}), 7.81-7.71(\mathrm{~m}, 1 \mathrm{H}), 7.67$ (br s, $1 \mathrm{H}), 7.61-7.54(\mathrm{~m}, 1 \mathrm{H})$, $7.31-7.38(\mathrm{~m}, 2 \mathrm{H}), 6.65(\mathrm{br} \mathrm{s}, 1 \mathrm{H}), 5.94(\mathrm{~d}, J=6.1 \mathrm{~Hz}, 1 \mathrm{H}), 4.26$ (br s, $1 \mathrm{H}), 1.24(\mathrm{~d}, J=6.5 \mathrm{~Hz}, 6 \mathrm{H}) .{ }^{13} \mathrm{C}$ NMR $(126 \mathrm{MHz}$, $\mathrm{MeOD}) \delta 162.44,159.53,153.19,151.87,140.94,133.84$, 129.47, 128.57, 118.84, 116.64, 101.95, 97.35.

$N^{2}$-(3-(1H-Imidazol-1-yl)phenyl)- $N^{4}$-isopropylpyrimidine2,4-diamine (6j). To a mixture of $3 f(0.10 \mathrm{mmol}, 1.0$ equiv $)$ in $\mathrm{EtOH}(0.50 \mathrm{~mL}, 0.20 \mathrm{M})$ under a nitrogen atmosphere at rt were added 3-( $1 \mathrm{H}$-imidazol-1-yl)aniline $(0.11 \mathrm{mmol}, 1.1$ equiv $)$ and a drop of $1 \mathrm{~N} \mathrm{HCl}$. The reaction mixture was heated to reflux and stirred at that temperature for $6 \mathrm{~h}$; it was then cooled to rt. Purification using automated flash chromatography $(\mathrm{MeOH} /$ $\mathrm{DCM}$ ) and then reversed-phase chromatography (MeCN/ water) was followed by evaporation, giving $\mathbf{6} \mathbf{j}$ as a colorless oil (0.004 g, 14\%). LC-MS (ESI) $m / z: 295[\mathrm{M}+\mathrm{H}]^{+} .{ }^{1} \mathrm{H}$ NMR $(500 \mathrm{MHz}, \mathrm{MeOD}) \delta 8.28$ (br s, $1 \mathrm{H}), 8.15$ (br s, $1 \mathrm{H}), 7.75$ (br s, $2 \mathrm{H}), 7.54-7.56(\mathrm{~m}, 1 \mathrm{H}), 7.48(\mathrm{t}, J=8.0 \mathrm{~Hz}, 1 \mathrm{H}), 7.45-7.09(\mathrm{~m}$, $2 \mathrm{H}), 6.07(\mathrm{~d}, J=5.8 \mathrm{~Hz}, 1 \mathrm{H}), 4.24(\mathrm{br} \mathrm{s}, 1 \mathrm{H}), 1.25(\mathrm{~d}, J=6.5 \mathrm{~Hz}$, $6 \mathrm{H}) .{ }^{13} \mathrm{C}$ NMR (126 MHz, MeOD) $\delta 162.22,156.66,147.86$, $140.93,137.76,129.90,118.82,115.17,112.80,98.35,42.50$, 21.05 .

$N^{4}$-Isopropyl- $N^{2}$-(3-(pyrazin-2-yl)phenyl)pyrimidine-2,4-diamine (6k). To a mixture of $3 \mathbf{f}(0.10 \mathrm{mmol}, 1.0$ equiv $)$ in $\mathrm{EtOH}$ $(0.50 \mathrm{~mL}, 0.20 \mathrm{M})$ under a nitrogen atmosphere at $\mathrm{rt}$ were added 3-(pyrazin-2-yl)aniline ( $0.11 \mathrm{mmol}, 1.1$ equiv) and a drop of $1 \mathrm{~N}$ $\mathrm{HCl}$. The reaction mixture was heated to reflux and stirred at that temperature for $3 \mathrm{~h}$; it was then cooled to rt. Purification using automated flash chromatography $(\mathrm{MeOH} / \mathrm{DCM})$ was followed by evaporation, giving $6 \mathrm{k}$ as a colorless foam $(0.011 \mathrm{~g}, 36 \%)$. TLC $R_{f} 0.5$ (10\% MeOH/DCM). LC-MS (ESI) $m / z: 307[\mathrm{M}+$ $\mathrm{H}]^{+} .{ }^{1} \mathrm{H}$ NMR (500 MHz, MeOD) $\delta 9.09(\mathrm{~d}, J=1.5 \mathrm{~Hz}, 1 \mathrm{H})$, $8.68(\mathrm{dd}, J=2.6,1.6 \mathrm{~Hz}, 1 \mathrm{H}), 8.63(\mathrm{~s}, 1 \mathrm{H}), 7.74(\mathrm{br} \mathrm{s}, 1 \mathrm{H}), 7.70-$ $7.61(\mathrm{~m}, 2 \mathrm{H}), 7.43(\mathrm{t}, J=7.9 \mathrm{~Hz}, 1 \mathrm{H}), 5.95(\mathrm{~d}, J=6.0 \mathrm{~Hz}, 1 \mathrm{H})$, 4.45-4.17 (m, 1H), $1.25(\mathrm{~d}, J=6.5 \mathrm{~Hz}, 6 \mathrm{H}) .{ }^{13} \mathrm{C}$ NMR (126 $\mathrm{MHz}, \mathrm{MeOD}) \delta 162.41,159.46,153.34,144.14,142.44,141.78$, $141.45,136.42,128.86,120.48,119.72,117.46,97.33,41.84$, 21.43.

$N^{4}$-isopropyl- $N^{2}$-(3-(pyridin-4-yl)phenyl)pyrimidine-2,4-diamine (6l). To a mixture of $3 f(0.10 \mathrm{mmol}, 1.0$ equiv $)$ in $\mathrm{EtOH}$ $(0.50 \mathrm{~mL}, 0.20 \mathrm{M})$ under a nitrogen atmosphere at rt were added 3-(4-pyridyl)aniline ( $0.11 \mathrm{mmol}, 1.1$ equiv) and a drop of $1 \mathrm{~N}$ $\mathrm{HCl}$. The reaction mixture was heated to reflux and stirred at that temperature for $3 \mathrm{~h}$; it was then cooled to rt. Purification using automated flash chromatography $(\mathrm{MeOH} / \mathrm{DCM})$ was followed by evaporation, giving 61 as an orange solid $(0.002 \mathrm{~g}, 7 \%)$. TLC $R_{f}$ $0.5(10 \% \mathrm{MeOH} / \mathrm{DCM})$. LC-MS (ESI) $m / z: 306[\mathrm{M}+\mathrm{H}]^{+} .{ }^{1} \mathrm{H}$ $\operatorname{NMR}\left(500 \mathrm{MHz}, \mathrm{CDCl}_{3}\right) \delta 8.74-8.59(\mathrm{~m}, 2 \mathrm{H}), 8.07($ br s, $1 \mathrm{H})$, $7.95(\mathrm{~d}, J=5.7 \mathrm{~Hz}, 1 \mathrm{H}), 7.64-7.58(\mathrm{~m}, 1 \mathrm{H}), 7.58-7.51(\mathrm{~m}, 2 \mathrm{H})$, $7.43(\mathrm{t}, J=7.9 \mathrm{~Hz}, 1 \mathrm{H}), 7.27(\mathrm{~s}, 1 \mathrm{H}), 7.14(\mathrm{br} \mathrm{s}, 1 \mathrm{H}), 5.88(\mathrm{~d}, J=$ $5.8 \mathrm{~Hz}, 1 \mathrm{H}), 4.69(\mathrm{br} \mathrm{s}, 1 \mathrm{H}), 4.10($ br s, $1 \mathrm{H}), 1.28(\mathrm{~d}, J=6.5 \mathrm{~Hz}$, $6 \mathrm{H}) .{ }^{13} \mathrm{C} \mathrm{NMR}\left(126 \mathrm{MHz}, \mathrm{CDCl}_{3}\right) \delta 162.12,159.56,155.58$, $150.15,148.67,140.88,138.85,129.48,121.79,120.44,119.58$, 117.51, 42.79, 22.85.

$N^{2}$-([1, 1'-Biphenyl]-4-yl)- $N^{4}$-isopropylpyrimidine-2,4-diamine $(6 \mathrm{~m})$. To a mixture of $3 \mathrm{f}(0.12 \mathrm{mmol}, 1.0$ equiv $)$ in EtOH $(0.50 \mathrm{~mL}, 0.23 \mathrm{M})$ under a nitrogen atmosphere at rt were added 4-aminobiphenyl ( $0.18 \mathrm{mmol}, 1.5$ equiv) and a drop of $1 \mathrm{~N} \mathrm{HCl}$. The reaction mixture was heated to reflux and stirred at that temperature for $2 \mathrm{~h}$; it was then cooled to rt. Purification using automated flash chromatography $(\mathrm{MeOH} / \mathrm{DCM})$ was followed by evaporation, giving $6 \mathrm{~m}$ as a white solid $(0.024 \mathrm{~g}, 68 \%)$. TLC $R_{f}$
$0.7(10 \% \mathrm{MeOH} / \mathrm{DCM}) . \mathrm{LC}-\mathrm{MS}$ (ESI) $m / z: 305[\mathrm{M}+\mathrm{H}]^{+} .{ }^{1} \mathrm{H}$ NMR (400 MHz, chloroform-d) $\delta 8.05$ (br s, $1 \mathrm{H}), 7.81$ (br s, $1 \mathrm{H}), 7.73-7.63(\mathrm{~m}, 2 \mathrm{H}), 7.63-7.48(\mathrm{~m}, 4 \mathrm{H}), 7.48-7.37(\mathrm{~m}$, $2 \mathrm{H}), 7.34-7.28(\mathrm{~m}, 1 \mathrm{H}), 5.93(\mathrm{~d}, J=6.2 \mathrm{~Hz}, 1 \mathrm{H}), 5.34(\mathrm{br} \mathrm{s}$, $1 \mathrm{H}), 4.07$ (br s, $1 \mathrm{H}), 1.28(\mathrm{~d}, J=6.5 \mathrm{~Hz}, 6 \mathrm{H}) .{ }^{13} \mathrm{C}$ NMR $(101$ $\left.\mathrm{MHz}, \mathrm{CDCl}_{3}\right) \delta 162.08,140.77,138.57,135.42,128.73,127.41$, 126.81, 126.72, 119.93, 43.25, 22.64.

4-((4-(Isopropylamino)pyrimidin-2-yl)amino)benzamide (6n). To a mixture of $3 \mathrm{f}(0.10 \mathrm{mmol}, 1.0$ equiv) in $\mathrm{EtOH}(0.50$ $\mathrm{mL}, 0.20 \mathrm{M})$ under a nitrogen atmosphere at rt were added 4aminobenzamide ( $0.15 \mathrm{mmol}, 1.5$ equiv) and a drop of $1 \mathrm{~N} \mathrm{HCl}$. The reaction mixture was heated to reflux and stirred at that temperature for $2 \mathrm{~h}$; it was then cooled to rt. Purification using automated flash chromatography $(\mathrm{MeOH} / \mathrm{DCM})$ was followed by evaporation, giving $6 \mathrm{n}$ as a white solid $(0.013 \mathrm{~g}, 48 \%)$. TLC $R_{f}$ $0.3(10 \% \mathrm{MeOH} / \mathrm{DCM})$. LC-MS (ESI) $m / z: 272[\mathrm{M}+\mathrm{H}]^{+} .{ }^{1} \mathrm{H}$ NMR (500 MHz, MeOD) $\delta 7.86-7.77(\mathrm{~m}, 5 \mathrm{H}), 5.99(\mathrm{~d}, J=6.1$ $\mathrm{Hz}, 1 \mathrm{H}), 4.23(\mathrm{~s}, 1 \mathrm{H}), 1.27(\mathrm{~d}, J=6.5 \mathrm{~Hz}, 6 \mathrm{H}) .{ }^{13} \mathrm{C}$ NMR $(126$ $\mathrm{MHz}, \mathrm{MeOD}) \delta 170.82,162.40,159.20,153.30,144.38,128.12$, 125.46, 117.72, 97.87, 41.96, 21.31.

Biology. Biochemical Assays. Briefly, kinase reactions were conducted in triplicate, with a final assay volume of $10 \mu \mathrm{L}$, in black polystyrene 384-well plates (Corning \#8849BC) using the Z'-LYTE Kinase Assay Kit (Tyr 2 peptide; Life Technologies). The reaction mixtures contained $500 \mu \mathrm{M}$ ATP, $2 \mu \mathrm{M}$ Tyr 2 peptide, and 6.25 or $12.50 \mathrm{nM}$ recombinant human FLT3 (571993 (SignalChem) in buffer [50 mM HEPES ( $\mathrm{pH} 7.5$ ), $10 \mathrm{mM}$ $\mathrm{MgCl}_{2}, 1 \mathrm{mM}$ EGTA, 0.01\% Brij-35, $\left.0.25 \mathrm{mM} \mathrm{DTT}\right]$ ). A cocktail of FLT3, ATP, and peptide solution prepared in the abovedescribed buffer $(10 \mu \mathrm{L})$ was added to the plates using an automated plate filler (Wellmate, Matrix). DMSO stock solutions of the test compounds were then added in a dilution series by pin transfer (V\&P Scientific) in nanoliter volumes. After $1 \mathrm{~h}$ of incubation at $23{ }^{\circ} \mathrm{C}$, the reaction mixtures were quenched and processed according to the $\mathrm{Z}^{\prime}$-LYTE kit protocol and read on an automated EnVision (Perkin-Elmer) plate reader using a 400 $\mathrm{nm}$ excitation filter and a $535 \mathrm{~nm}$ emission filter. Fluorescence emission ratio data were processed using a custom script written in Pipeline Pilot (Accelrys) to transform data by $\log _{10}$ and calculate percent inhibition normalized in comparison to the means of positive and negative controls. Dose-response curves were plotted using GraphPad Prism software.

MV4-11 and BJ Cell Culture and Cytotoxicity Studies. BJ and MV4-11 cells were purchased from the American Type Culture Collection (ATCC, Manassas, Virginia). The cells were cultured in a humidified $5 \% \mathrm{CO}_{2}$ incubator at $37^{\circ} \mathrm{C}$ according to vendor recommendations. BJ cells are cultured in EMEM media and MV4-11 cells are cultured in IMDM media purchased from ATCC; both lines are supplemented with $10 \%$ fetal bovine serum (GE Healthcare Life Sciences, Hyclone Laboratories, Logan, Utah). The cells were routinely tested for mycoplasma with the MycoAlert Mycoplasma Detection Kit (Lonza, Walkersville, $\mathrm{MD})$.

Approximately 1000 BJ or 500 MV4-11 exponentially growing cells were plated per well $(30 \mu \mathrm{L})$ in white polystyrene flatbottom sterile 384-well tissue-culture-treated plates (Corning, Tewksbury, MA) and incubated overnight at $37{ }^{\circ} \mathrm{C}$ in a humidified $5 \% \mathrm{CO}_{2}$ incubator. Compound solutions (DMSO) were pin-transferred (V\&P Scientific, San Diego, CA) the following day. Inhibition of proliferation was determined following $72 \mathrm{~h}$ of incubation using Promega Cell Titer Glo Reagent according to the manufacturer's recommendations. 
Luminescence was measured on an Envision plate reader (Perkin-Elmer).

MOLM13 Cell Culture. Parental MOLM13 ${ }^{\text {FLT3-ITD }}$ cells harboring FLT3-ITD (DSMZ, Brunswick, Germany) and MOLM13 $3^{\text {FLT3-ITD/D835Y cells carrying an additional D835Y }}$ mutation were maintained in RPMI 1640 medium supplemented with $10 \%$ fetal bovine serum (Life Technologies, Grand Island, $\mathrm{NY}$ ) in a humidified $5 \% \mathrm{CO}_{2}$ incubator at $37{ }^{\circ} \mathrm{C}$.,50 The MOLM13 $3^{\text {FLT3-ITD/D835Y }}$ cells were maintained in the presence of $5 \mu \mathrm{M}$ tandutinib (LC Laboratories, Woburn, MA) and removed from the presence of the drug 3 days prior to experimentation

MOLM13 Cell Viability. Cell viability upon drug treatment was assessed using the MTT reagent (Roche Applied Science, Indianapolis, IN), as previously described. ${ }^{50}$ Briefly, the cells were plated overnight in a humidified $5 \% \mathrm{CO}_{2}$ incubator at 37 ${ }^{\circ} \mathrm{C}$. On day 2, the cells were treated with DMSO or drug reconstituted in DMSO for $72 \mathrm{~h}$. The drug concentrations used were as follows: compound 1, 1000-167 nM; compound 5e 1600-6.55 nM; compound 6a, 1000-6.55 nM; and compound 6k, 1500-0.98 nM. Three individual experiments were conducted, with six replicates each. Cell viability was quantified as relative percentage to DMSO-treated cells, and cell viability curves were generated using GraphPad Prism software version 6.07 .

Western Blot Analysis. Whole-cell lysates were made with radioimmunoprecipitation assay buffer $(20 \mathrm{mM}$ Tris- $\mathrm{HCl}(\mathrm{pH}$ 7.5), $150 \mathrm{mM} \mathrm{NaCl}, 1 \mathrm{mM} \mathrm{Na}{ }_{2}$ EDTA, 1 mM EGTA, 1\% NP-40, $1 \%$ sodium deoxycholate, $0.1 \%$ SDS, and protease and phosphatase inhibitors). Immunoprecipitation was carried out with anti-FLT3 antibody and Protein A/G agarose beads from Santa Cruz Biotechnology (Dallas, TX). Invitrogen Bis-trisgradient mini or midi gels were used for western blot analysis, followed by detection with enhanced chemiluminescence (ECL) reagent. Primary antibodies used were from Cell Signaling Technology (Danvers, MA): FLT3, phospho-FLT3, STAT5, and phospho-STAT5. Secondary antibodies were from Jackson Immunoresearch and Cell Signaling Technology.

In Vivo Pharmacokinetic Study. All animal studies were carried out under a St Jude Children's Research Hospital IACUC-approved protocol (\#477). Female C57BL/6 mice, with an average weight of $18 \mathrm{~g}$, were purchased from Charles River Laboratories (Wilmington, MA). Food and water were provided ad libitum. Twenty-four mice were divided into four dosage groups: $0,3,5$, and $10 \mathrm{mg} / \mathrm{kg}$. For each mouse, $0.1 \mathrm{~mL}$ of the compound suspension in formulation (5:50:45, EtOH/ PEG400/PBS ( $\mathrm{pH} 7.4), \mathrm{v} / \mathrm{v} / \mathrm{v})$ was administered by i.p. injection. Blood $(0.1 \mathrm{~mL})$ was collected retro-orbitally from a different mouse within each dosage group at 5, 15, and $30 \mathrm{~min}$ and 1,4 , and $24 \mathrm{~h}$. The animals were euthanized via cardiac puncture after anesthesia at $48 \mathrm{~h}$ post injection. The blood samples were treated with $10 \mu \mathrm{L}$ of EDTA sodium solution to prevent coagulation. The blood samples were kept under ice and centrifuged for $3 \mathrm{~min}$ at $13200 \mathrm{rpm}$ in a desktop centrifuge to collect plasma. The plasma samples $(25 \mu \mathrm{L})$ were combined with $75 \mu \mathrm{L}$ of internal standard ( $2 \mu \mathrm{M}$ warfarin) in acetonitrile in a $96-$ well plate and centrifuged at $4000 \mathrm{rpm}$ for $20 \mathrm{~min}$ at $4{ }^{\circ} \mathrm{C}$. The supernatant $(40 \mu \mathrm{L})$ was collected and mixed with two parts of deionized water and centrifuged again at $4000 \mathrm{rpm}$ for $20 \mathrm{~min}$ at $4{ }^{\circ} \mathrm{C}$. The plasma concentration was determined using an LC/ MS-MS assay with multiple reaction monitoring detection ( $\mathrm{AB}$ Sciex, Framingham, MA). The LC/MS-MS method is provided in the Supporting Information. The assay limit of quantification (LLOQ) was $13.7 \mathrm{nM}$ in plasma.
The processed plasma concentration-time data were analyzed using noncompartmental analysis (NCA) in WinNonlin 6.0 with the Plasma (200-202) model type; all standard NCA parameters were estimated via default software settings, using predicted parameter estimates. If at least two-thirds of the observed concentrations were below the LLOQ the mean concentration was treated as missing.

The AUC was calculated with the linear trapezoidal, linear interpolation rule using mean concentrations and nominal times. The terminal elimination rate (Lambda_z) and half-life (HL_Lambda_z) were determined using the default "Best Fit" method. The predicted AUC from the last time point to infinity (AUCINF pred) was calculated as AUClast plus Clast(pred)/ Lambda_z. CL was calculated as Dose/AUCINF_pred.

\section{ASSOCIATED CONTENT}

\section{Supporting Information}

The Supporting Information is available free of charge on the ACS Publications website at DOI: 10.1021/acsomega.7b00144.

Experimental procedures for ADME and MTD studies; additional experimental procedures for synthesis and characterization of compounds; additional synthetic schemes for preparation of intermediates; KinomeScan analysis for compound 1; growth inhibition data against the BJ cell line for all compounds; FLT3 inhibition and MV4-11 cell proliferation data, including CI 95; solubility and permeability data for selected compounds; cellular permeability and PGP efflux assay data; in vivo blood chemistry data after i.p. dosing of compound $5 \mathbf{e}$ in mice (PDF)

Experimental data for compounds 1, 4-6, 9, 13, 14, 16, 17, and 19-22 (XLSX)

\section{AUTHOR INFORMATION}

\section{Corresponding Author}

*E-mail: kip.guy@uky.edu. Phone: 859-257-5290. Fax: 859-2572128.

ORCID

R. Kiplin Guy: 0000-0002-9638-2060

Present Address

${ }^{\S}$ Department of Pharmaceutical Sciences, University of Kentucky College of Pharmacy, 214H BioPharm Complex, Lexington, Kentucky 40536, United States (R.K.G. and Y.C.)

\section{Author Contributions}

The manuscript was prepared through contributions of all authors.

Notes

The authors declare no competing financial interest.

\section{ACKNOWLEDGMENTS}

This work was supported by the Department of Defense (CA093469P2), NCI (CA138744-07), St. Jude Children's Research Hospital, and the American Lebanese Syrian Associated Charities (ALSAC).

\section{ABBREVIATIONS}

FLT3, FMS-like tyrosine kinase; SAR, structure-activity relationship; AML, acute myelogenous leukemia; FL, FLT3 ligand; ITD, internal tandem duplication; JM, juxtamembrane; wt, wild-type; DFG, Asp-Phe-Gly; SPR, structure-property relationship; CI, confidence interval; PGP, P-glycoprotein; TKD, 
tyrosine kinase domain; i.p, intraperitoneal; DCM, dichloromethane; EtOAc, ethyl acetate; $\mathrm{EtOH}$, ethanol; $\mathrm{MeOH}$, methanol

\section{REFERENCES}

(1) Wander, S. A.; Levis, M. J.; Fathi, A. T. The evolving role of FLT3 inhibitors in acute myeloid leukemia: quizartinib and beyond. Ther. Adv. Hematol. 2014, 5, 65-77.

(2) Takahashi, S. Downstream molecular pathways of FLT3 in the pathogenesis of acute myeloid leukemia: biology and therapeutic implications. J. Hematol. Oncol. 2011, 4, 13.

(3) Gilliland, D. G.; Griffin, J. D. The roles of FLT3 in hematopoiesis and leukemia. Blood 2002, 100, 1532-1542.

(4) deLapeyrière, O.; Naquet, P.; Planche, J.; Marchetto, S.; Rottapel, R.; Gambarelli, D.; Rosnet, O.; Birnbaum, D. Expression of Flt3 Tyrosine Kinase Receptor Gene in Mouse Hematopoietic and Nervous Tissues. Differentiation 1995, 58, 351-359.

(5) Dosil, M.; Wang, S.; Lemischka, I. R. Mitogenic signalling and substrate specificity of the Flk2/Flt3 receptor tyrosine kinase in fibroblasts and interleukin 3-dependent hematopoietic cells. Mol. Cell. Biol. 1993, 13, 6572-6585.

(6) Hayakawa, F.; Towatari, M.; Kiyoi, H.; Tanimoto, M.; Kitamura, T.; Saito, H.; Naoe, T. Tandem-duplicated Flt3 constitutively activates STAT5 and MAP kinase and introduces autonomous cell growth in IL3-dependent cell lines. Oncogene 2000, 19, 624-631.

(7) Mizuki, M.; Fenski, R.; Halfter, H.; Matsumura, I.; Schmidt, R.; Muller, C.; Gruning, W.; Kratz-Albers, K.; Serve, S.; Steur, C.; Buchner, T.; Kienast, J.; Kanakura, Y.; Berdel, W. E.; Serve, H. Flt3 mutations from patients with acute myeloid leukemia induce transformation of 32D cells mediated by the Ras and STAT5 pathways. Blood 2000, 96, 3907-3914.

(8) Zhang, S.; Broxmeyer, H. E. p85 subunit of PI3 kinase does not bind to human Flt3 receptor, but associates with SHP2, SHIP, and a tyrosine-phosphorylated $100-\mathrm{kDa}$ protein in Flt3 ligand-stimulated hematopoietic cells. Biochem. Biophys. Res. Commun. 1999, 254, 440445.

(9) Yokota, S.; Kiyoi, H.; Nakao, M.; Iwai, T.; Misawa, S.; Okuda, T.; Sonoda, Y.; Abe, T.; Kahsima, K.; Matsuo, Y.; Naoe, T. Internal tandem duplication of the FLT3 gene is preferentially seen in acute myeloid leukemia and myelodysplastic syndrome among various hematological malignancies. A study on a large series of patients and cell lines. Leukemia 1997, 11, 1605-1609.

(10) Kiyoi, H.; Naoe, T.; Yokota, S.; Nakao, M.; Minami, S.; Kuriyama, K.; Takeshita, A.; Saito, K.; Hasegawa, S.; Shimodaira, S.; Tamura, J.; Shimazaki, C.; Matsue, K.; Kobayashi, H.; Arima, N.; Suzuki, R.; Morishita, H.; Saito, H.; Ueda, R.; Ohno, R. Internal tandem duplication of FLT3 associated with leukocytosis in acute promyelocytic leukemia. Leukemia Study Group of the Ministry of Health and Welfare (Kohseisho). Leukemia 1997, 11, 1447-1452.

(11) Yamamoto, Y.; Kiyoi, H.; Nakano, Y.; Suzuki, R.; Kodera, Y.; Miyawaki, S.; Asou, N.; Kuriyama, K.; Yagasaki, F.; Shimazaki, C.; Akiyama, H.; Saito, K.; Nishimura, M.; Motoji, T.; Shinagawa, K.; Takeshita, A.; Saito, H.; Ueda, R.; Ohno, R.; Naoe, T. Activating mutation of D835 within the activation loop of FLT3 in human hematologic malignancies. Blood 2001, 97, 2434-2439.

(12) Nakao, M.; Yokota, S.; Iwai, T.; Kaneko, H.; Horiike, S.; Kashima, K.; Sonoda, Y.; Fujimoto, T.; Misawa, S. Internal tandem duplication of the flt3 gene found in acute myeloid leukemia. Leukemia 1996, 10, 1911-1918.

(13) Abu-Duhier, F. M.; Goodeve, A. C.; Wilson, G. A.; Care, R. S.; Peake, I. R.; Reilly, J. T. Identification of novel FLT-3 Asp835 mutations in adult acute myeloid leukaemia. Br. J. Haematol. 2001, 113, 983-988.

(14) Taketani, T.; Taki, T.; Sugita, K.; Furuichi, Y.; Ishii, E.; Hanada, R.; Tsuchida, M.; Sugita, K.; Ida, K.; Hayashi, Y. FLT3 mutations in the activation loop of tyrosine kinase domain are frequently found in infant ALL with MLL rearrangements and pediatric ALL with hyperdiploidy. Blood 2004, 103, 1085-1088.
(15) Cloos, J.; Goemans, B. F.; Hess, C. J.; van Oostveen, J. W.; Waisfisz, Q.; Corthals, S.; de Lange, D.; Boeckx, N.; Hahlen, K.; Reinhardt, D.; Creutzig, U.; Schuurhuis, G. J.; Zwaan, C. M.; Kaspers, G. $\mathrm{J}$. Stability and prognostic influence of FLT3 mutations in paired initial and relapsed AML samples. Leukemia 2006, 20, 1217-1220.

(16) Meshinchi, S.; Appelbaum, F. R. Structural and functional alterations of FLT3 in acute myeloid leukemia. Clin. Cancer Res. 2009, $15,4263-4269$

(17) Stone, R. M.; DeAngelo, D. J.; Klimek, V.; Galinsky, I.; Estey, E.; Nimer, S. D.; Grandin, W.; Lebwohl, D.; Wang, Y.; Cohen, P.; Fox, E. A.; Neuberg, D.; Clark, J.; Gilliland, D. G.; Griffin, J. D. Patients with acute myeloid leukemia and an activating mutation in FLT3 respond to a small-molecule FLT3 tyrosine kinase inhibitor, PKC412. Blood 2005, $105,54-60$.

(18) Stone, R. M.; Fischer, T.; Paquette, R.; Schiller, G.; Schiffer, C. A.; Ehninger, G.; Cortes, J.; Kantarjian, H. M.; DeAngelo, D. J.; HuntsmanLabed, A.; Dutreix, C.; del Corral, A.; Giles, F. Phase IB study of the FLT3 kinase inhibitor midostaurin with chemotherapy in younger newly diagnosed adult patients with acute myeloid leukemia. Leukemia 2012, 26, 2061-2068

(19) Fischer, T.; Stone, R. M.; Deangelo, D. J.; Galinsky, I.; Estey, E.; Lanza, C.; Fox, E.; Ehninger, G.; Feldman, E. J.; Schiller, G. J.; Klimek, V. M.; Nimer, S. D.; Gilliland, D. G.; Dutreix, C.; Huntsman-Labed, A.; Virkus, J.; Giles, F. J. Phase IIB trial of oral Midostaurin (PKC412), the FMS-like tyrosine kinase 3 receptor (FLT3) and multi-targeted kinase inhibitor, in patients with acute myeloid leukemia and high-risk myelodysplastic syndrome with either wild-type or mutated FLT3. J. Clin. Oncol. 2010, 28, 4339-4345.

(20) Smith, B. D.; Levis, M.; Beran, M.; Giles, F.; Kantarjian, H.; Berg, K.; Murphy, K. M.; Dauses, T.; Allebach, J.; Small, D. Single-agent CEP701, a novel FLT3 inhibitor, shows biologic and clinical activity in patients with relapsed or refractory acute myeloid leukemia. Blood 2004, 103, 3669-3676.

(21) Knapper, S.; Burnett, A. K.; Littlewood, T.; Kell, W. J.; Agrawal, S.; Chopra, R.; Clark, R.; Levis, M. J.; Small, D. A phase 2 trial of the FLT3 inhibitor lestaurtinib (CEP701) as first-line treatment for older patients with acute myeloid leukemia not considered fit for intensive chemotherapy. Blood 2006, 108, 3262-3270.

(22) Levis, M.; Ravandi, F.; Wang, E. S.; Baer, M. R.; Perl, A.; Coutre, S.; Erba, H.; Stuart, R. K.; Baccarani, M.; Cripe, L. D.; Tallman, M. S.; Meloni, G.; Godley, L. A.; Langston, A. A.; Amadori, S.; Lewis, I. D.; Nagler, A.; Stone, R.; Yee, K.; Advani, A.; Douer, D.; Wiktor-Jedrzejczak, W.; Juliusson, G.; Litzow, M. R.; Petersdorf, S.; Sanz, M.; Kantarjian, H. M.; Sato, T.; Tremmel, L.; Bensen-Kennedy, D. M.; Small, D.; Smith, B. D. Results from a randomized trial of salvage chemotherapy followed by lestaurtinib for patients with FLT3 mutant AML in first relapse. Blood 2011, 117, 3294-3301.

(23) Smith, C. C.; Lasater, E. A.; Lin, K. C.; Wang, Q.; McCreery, M. Q.; Stewart, W. K.; Damon, L. E.; Perl, A. E.; Jeschke, G. R.; Sugita, M.; Carroll, M.; Kogan, S. C.; Kuriyan, J.; Shah, N. P. Crenolanib is a selective type I pan-FLT3 inhibitor. Proc. Natl. Acad. Sci. U.S.A. 2014, $111,5319-5324$.

(24) Lewis, N. L.; Lewis, L. D.; Eder, J. P.; Reddy, N. J.; Guo, F.; Pierce, K. J.; Olszanski, A. J.; Cohen, R. B. Phase I study of the safety, tolerability, and pharmacokinetics of oral CP-868,596, a highly specific platelet-derived growth factor receptor tyrosine kinase inhibitor in patients with advanced cancers. J. Clin. Oncol. 2009, 27, 5262-5269.

(25) Michael, M.; Vlahovic, G.; Khamly, K.; Pierce, K. J.; Guo, F.; Olszanski, A. J. Phase Ib study of CP-868,596, a PDGFR inhibitor, combined with docetaxel with or without axitinib, a VEGFR inhibitor. Br. J. Cancer 2010, 103, 1554-1561.

(26) Cortes, J.; Kantarjian, H. How I treat newly diagnosed chronic phase CML. Blood 2012, 120, 1390-1397.

(27) DeAngelo, D. J.; Stone, R. M.; Heaney, M. L.; Nimer, S. D.; Paquette, R. L.; Klisovic, R. B.; Caligiuri, M. A.; Cooper, M. R.; Lecerf, J. M.; Karol, M. D.; Sheng, S.; Holford, N.; Curtin, P. T.; Druker, B. J.; Heinrich, M. C. Phase 1 clinical results with tandutinib (MLN518), a novel FLT3 antagonist, in patients with acute myelogenous leukemia or 
high-risk myelodysplastic syndrome: safety, pharmacokinetics, and pharmacodynamics. Blood 2006, 108, 3674-3681.

(28) De Angelo, D. J.; Stone, R. M.; Heaney, M. L.; Nimer, S. D.; Paquette, R.; Bruner-Klisovic, R.; Caligiuri, M. A.; Cooper, M. R.; LeCerf, J. M.; Iyer, G. Phase II evaluation of the tyrosine kinase inhibitor MLN518 in patients with acute myeloid leukemia (AML) bearing a FLT3 internal tandem duplication (ITD) mutation. Blood 2004, 104, 496 .

(29) Knapper, S.; White, P.; Levis, M. J.; Hills, R. K.; Russell, N. H.; Burnett, A. The Efficacy of the FLT3 Inhibitor Lestaurtinib in AML Depends on Adequate Plasma Inhibitory Activity (PIA), and Is Unaffected by Rising FLT Ligand Levels: An Update of the NCRI AML15 \& 17 Trials. Blood 2011, 118, 194.

(30) O’Farrell, A. M.; Foran, J. M.; Fiedler, W.; Serve, H.; Paquette, R. L.; Cooper, M. A.; Yuen, H. A.; Louie, S. G.; Kim, H.; Nicholas, S.; Heinrich, M. C.; Berdel, W. E.; Bello, C.; Jacobs, M.; Scigalla, P.; Manning, W. C.; Kelsey, S.; Cherrington, J. M. An innovative phase I clinical study demonstrates inhibition of FLT3 phosphorylation by SU11248 in acute myeloid leukemia patients. Clin. Cancer Res. 2003, 9, $5465-5476$.

(31) Fiedler, W.; Serve, H.; Dohner, H.; Schwittay, M.; Ottmann, O. G.; O'Farrell, A. M.; Bello, C. L.; Allred, R.; Manning, W. C.; Cherrington, J. M.; Louie, S. G.; Hong, W.; Brega, N. M.; Massimini, G.; Scigalla, P.; Berdel, W. E.; Hossfeld, D. K. A phase 1 study of SU11248 in the treatment of patients with refractory or resistant acute myeloid leukemia (AML) or not amenable to conventional therapy for the disease. Blood 2005, 105, 986-993.

(32) Fiedler, W.; Kayser, S.; Kebenko, M.; Krauter, J.; Salih, H. R.; Gotze, K.; Spath, D.; Gohring, G.; Teleanu, V.; Dohner, K.; Ganser, A.; Dohner, H.; Schlenk, R. F. Sunitinib and Intensive Chemotherapy in Patients with Acute Myeloid Leukemia and Activating FLT3 Mutations: Results of the AMLSG 10-07 Study (ClinicalTrials.gov No. NCT00783653). Blood 2012, 120, 1483.

(33) Zhang, W.; Konopleva, M.; Shi, Y. X.; McQueen, T.; Harris, D.; Ling, X. Y.; Estrov, Z.; Quintas-Cardama, A.; Small, D.; Cortes, J.; Andreeff, M. Mutant FLT3: A direct target of sorafenib in acute myelogenous leukemia. J. Natl. Cancer Inst. 2008, 100, 184-198.

(34) Metzelder, S. K.; Schroeder, T.; Finck, A.; Scholl, S.; Fey, M.; Gotze, K.; Linn, Y. C.; Kroger, M.; Reiter, A.; Salih, H. R; Heinicke, T.; Stuhlmann, R.; Muller, L.; Giagounidis, A.; Meyer, R. G.; Brugger, W.; Vohringer, M.; Dreger, P.; Mori, M.; Basara, N.; Schafer-Eckart, K.; Schultheis, B.; Baldus, C.; Neubauer, A.; Burchert, A. High activity of sorafenib in FLT3-ITD-positive acute myeloid leukemia synergizes with allo-immune effects to induce sustained responses. Leukemia 2012, 26, 2353-2359.

(35) Pratz, K. W.; Cho, E.; Levis, M. J.; Karp, J. E.; Gore, S. D.; McDevitt, M.; Stine, A.; Zhao, M.; Baker, S. D.; Carducci, M. A.; Wright, J. J.; Rudek, M. A.; Smith, B. D. A pharmacodynamic study of sorafenib in patients with relapsed and refractory acute leukemias. Leukemia 2010, 24, 1437-1444.

(36) Ravandi, F.; Cortes, J. E.; Jones, D.; Faderl, S.; Garcia-Manero, G.; Konopleva, M. Y.; O’Brien, S.; Estrov, Z.; Borthakur, G.; Thomas, D.; Pierce, S. R.; Brandt, M.; Byrd, A.; Bekele, B. N.; Pratz, K.; Luthra, R.; Levis, M.; Andreeff, M.; Kantarjian, H. M. Phase I/II Study of Combination Therapy With Sorafenib, Idarubicin, and Cytarabine in Younger Patients With Acute Myeloid Leukemia. J. Clin. Oncol. 2010, 28, 1856-1862.

(37) Grunwald, M. R.; Levis, M. J. FLT3 inhibitors for acute myeloid leukemia: a review of their efficacy and mechanisms of resistance. Int. J. Hematol. 2013, 97, 683-694.

(38) Randhawa, J. K.; Kantarjian, H. M.; Borthakur, G.; Thompson, P. A.; Konopleva, M.; Daver, N.; Pemmaraju, N.; Jabbour, E.; Kadia, T. M.; Estrov, Z.; Ramachandran, A.; Paradela, J.; Andreef, M.; Levis, M.; Ravandi, F.; Cortes, J. E. Results of a Phase II Study of Crenolanib in Relapsed/Refractory Acute Myeloid Leukemia Patients (Pts) with Activating FLT3 Mutations. Blood 2014, 124, 389.

(39) Zarrinkar, P. P.; Gunawardane, R. N.; Cramer, M. D.; Gardner, M. F.; Brigham, D.; Belli, B.; Karaman, M. W.; Pratz, K. W.; Pallares, G.; Chao, Q.; Sprankle, K. G.; Patel, H. K.; Levis, M.; Armstrong, R. C.;
James, J.; Bhagwat, S. S. AC220 is a uniquely potent and selective inhibitor of FLT3 for the treatment of acute myeloid leukemia (AML). Blood 2009, 114, 2984-92.

(40) Zarrinkar, P.; Armstrong, R.; Gunawardane, R.; James, J.; Karaman, M.; Bhagwat, S. Characterization of the biochemical and cellular activity of the second-generation FLT3 inhibitor AC220. Cancer Res. 2009, 69, No. 1787.

(41) Cortes, J. E.; Perl, A. E.; Dombret, H.; Kayser, S.; Steffen, B.; Rousselot, P.; Martinelli, G.; Estey, E. H.; Burnett, A. K.; Gammon, G.; Trone, D.; Leo, E.; Levis, M. J. Final Results of a Phase 2 Open-Label, Monotherapy Efficacy and Safety Study of Quizartinib (AC220) in Patients $\geq 60$ Years of Age with FLT3 ITD Positive or Negative Relapsed/Refractory Acute Myeloid Leukemia. Blood 2012, 120, 48.

(42) Cortes, J. E.; Kantarjian, H. M.; Foran, J. M.; Ghirdaladze, D.; Zodelava, M.; Borthakur, G.; Gammon, G.; Trone, D.; Armstrong, R. C.; James, J.; Levis, M. J. Final Results of A Phase 1 Study Investigating the Safety, Tolerability, Pharmacokinetics, and Pharmacodynamics of Quizartinib (AC220) Administered Daily to Patients with Relapsed or Refractory Acute Myeloid Leukemia Irrespective of FLT3-ITD Status. Blood 2012, 120, 1507.

(43) Kindler, T.; Lipka, D. B.; Fischer, T. FLT3 as a therapeutic target in AML: still challenging after all these years. Blood 2010, 116, 50895102.

(44) Weisberg, E.; Sattler, M.; Ray, A.; Griffin, J. D. Drug resistance in mutant FLT3-positive AML. Oncogene 2010, 29, 5120-5134.

(45) Baker, S. D.; Zimmerman, E. I.; Wang, Y.D.; Orwick, S.; Zatechka, D. S.; Buaboonnam, J.; Neale, G. A.; Olsen, S. R.; Enemark, E. J.; Shurtleff, S.; Rubnitz, J. E.; Mullighan, C. G.; Inaba, H. Emergence of polyclonal FLT3 tyrosine kinase domain mutations during sequential therapy with sorafenib and sunitinib in FLT3-ITD-positive acute myeloid leukemia. Clin. Cancer Res. 2013, 19, 5758-68.

(46) Smith, C. C.; Lin, K.; Stecula, A.; Sali, A.; Shah, N. P. FLT3 D835 mutations confer differential resistance to type II FLT3 inhibitors. Leukemia 2015, 29, 2390-2392.

(47) Smith, C. C.; Zhang, C.; Lin, K. C.; Lasater, E. A.; Zhang, Y.; Massi, E.; Damon, L. E.; Pendleton, M.; Bashir, A.; Sebra, R.; Perl, A.; Kasarskis, A.; Shellooe, R.; Tsang, G.; Carias, H.; Powell, B.; Burton, E. A.; Matusow, B.; Zhang, J.; Spevak, W.; Ibrahim, P. N.; Le, M. H.; Hsu, H. H.; Habets, G.; West, B. L.; Bollag, G.; Shah, N. P. Characterizing and Overriding the Structural Mechanism of the Quizartinib-Resistant FLT3 "Gatekeeper" F691L Mutation with PLX3397. Cancer Discovery 2015, 5, 668-679.

(48) Zorn, J. A.; Wang, Q.; Fujimura, E.; Barros, T.; Kuriyan, J. Crystal Structure of the FLT3 Kinase Domain Bound to the Inhibitor Quizartinib (AC220). PLoS One 2015, 10, No. e0121177.

(49) von Bubnoff, N.; Engh, R. A.; Aberg, E.; Sanger, J.; Peschel, C.; Duyster, J. FMS-like tyrosine kinase 3-internal tandem duplication tyrosine kinase inhibitors display a nonoverlapping profile of resistance mutations in vitro. Cancer Res. 2009, 69, 3032-3041.

(50) Zimmerman, E. I.; Turner, D. C.; Buaboonnam, J.; Hu, S.; Orwick, S.; Roberts, M. S.; Janke, L. J.; Ramachandran, A.; Stewart, C. F.; Inaba, H.; Baker, S. D. Crenolanib is active against models of drug-resistant FLT3-ITD-positive acute myeloid leukemia. Blood 2013, 122, 36073615.

(51) Kinase Profiling Service: DiscoveRx KINOMEscan. http://www. discoverx.com/home.

(52) Fabian, M. A.; Biggs, W. H., 3rd; Treiber, D. K.; Atteridge, C. E.; Azimioara, M. D.; Benedetti, M. G.; Carter, T. A.; Ciceri, P.; Edeen, P. T.; Floyd, M.; Ford, J. M.; Galvin, M.; Gerlach, J. L.; Grotzfeld, R. M.; Herrgard, S.; Insko, D. E.; Insko, M. A.; Lai, A. G.; Lelias, J. M.; Mehta, S. A.; Milanov, Z. V.; Velasco, A. M.; Wodicka, L. M.; Patel, H. K.; Zarrinkar, P. P.; Lockhart, D. J. A small molecule-kinase interaction map for clinical kinase inhibitors. Nat. Biotechnol. 2005, 23, 329-336.

(53) Karaman, M. W.; Herrgard, S.; Treiber, D. K.; Gallant, P.; Atteridge, C. E.; Campbell, B. T.; Chan, K. W.; Ciceri, P.; Davis, M. I.; Edeen, P. T.; Faraoni, R.; Floyd, M.; Hunt, J. P.; Lockhart, D. J.; Milanov, Z. V.; Morrison, M. J.; Pallares, G.; Patel, H. K.; Pritchard, S.; Wodicka, L. M.; Zarrinkar, P. P. A quantitative analysis of kinase inhibitor selectivity. Nat. Biotechnol. 2008, 26, 127-132. 
(54) Davis, M. I.; Hunt, J. P.; Herrgard, S.; Ciceri, P.; Wodicka, L. M.; Pallares, G.; Hocker, M.; Treiber, D. K.; Zarrinkar, P. P. Comprehensive analysis of kinase inhibitor selectivity. Nat. Biotechnol. 2011, 29, 10461051.

(55) Z'LYTE Assay Kits: ThermoFisher. https://www.thermofisher. $\mathrm{com} / \mathrm{us} / \mathrm{en} / \mathrm{home} /$ industrial/pharma-biopharma/drug-discoverydevelopment/target-and-lead-identification-and-validation/ kinasebiology/kinase-activity-assays/z-lyte.html.

(56) Fabbro, D.; Ruetz, S.; Buchdunger, E.; Cowan-Jacob, S. W.; Fendrich, G.; Liebetanz, J.; Mestan, J.; O’Reilly, T.; Traxler, P.; Chaudhuri, B.; Fretz, H.; Zimmermann, J.; Meyer, T.; Caravatti, G.; Furet, P.; Manley, P. W. Protein kinases as targets for anticancer agents: from inhibitors to useful drugs. Pharmacol. Ther. 2002, 93, 79-98.

(57) Di Gion, P.; Kanefendt, F.; Lindauer, A.; Scheffler, M.; Doroshyenko, O.; Fuhr, U.; Wolf, J.; Jaehde, U. Clinical Pharmacokinetics of Tyrosine Kinase Inhibitors Focus on Pyrimidines, Pyridines and Pyrroles. Clin. Pharmacokinet. 2011, 50, 551-603.

(58) Lawrence, H. R.; Martin, M. P.; Luo, Y.; Pireddu, R.; Yang, H.; Gevariya, H.; Ozcan, S.; Zhu, J. Y.; Kendig, R.; Rodriguez, M.; Elias, R.; Cheng, J. Q.; Sebti, S. M.; Schonbrunn, E.; Lawrence, N. J. Development of o-chlorophenyl substituted pyrimidines as exceptionally potent aurora kinase inhibitors. J. Med. Chem. 2012, 55, 7392-7416.

(59) Albrecht, F.; Sowada, O.; Fistikci, M.; Boysen, M. M. K. Heteroarylboronates in Rhodium-Catalyzed 1,4-Addition to Enones. Org. Lett. 2014, 16, 5212-5215.

(60) Bhattacharyya, S.; Neidigh, K. A.; Avery, M. A.; Williamson, J. S. Selective monoalkylation of ammonia: A high throughput synthesis of primary amines. Synlett 1999, 1999, 1781-1783.

(61) Liu, X.; Wright, M.; Hop, C. E. Rational use of plasma protein and tissue binding data in drug design. J. Med. Chem. 2014, 57, 8238-8248.

(62) Kinase $K_{\mathrm{d}}$ Determination: DiscoveRx KdELECT. http://www. discoverx.com/home.

(63) Wodicka, L. M.; Ciceri, P.; Davis, M. I.; Hunt, J. P.; Floyd, M.; Salerno, S.; Hua, X. H.; Ford, J. M.; Armstrong, R. C.; Zarrinkar, P. P.; Treiber, D. K. Activation state-dependent binding of small molecule kinase inhibitors: structural insights from biochemistry. Chem. Biol. 2010, 17, 1241-1249.

(64) Pangborn, A. B.; Giardello, M. A.; Grubbs, R. H.; Rosen, R. K.; Timmers, F. J. Safe and convenient procedure for solvent purification. Organometallics 1996, 15, 1518-1520.

(65) Still, W. C.; Kahn, M.; Mitra, A. Rapid Chromatographic Technique for Preparative Separations with Moderate Resolution. J. Org. Chem. 1978, 43, 2923-2925. 